\title{
ELECTROMAGNETIC AND MECHANICAL DESIGN OF GRIDDED RADIO-FREQUENCY CAVITY WINDOWS
}

\author{
BY \\ MOHAMMAD M. ALSHARO'A
}

\author{
Submitted in partial fulfillment of the \\ requirements for the degree of \\ Doctor of Philosophy in Mechanical Engineering \\ in the Graduate College of the \\ Illinois Institute of Technology
}

Approved

Advisor

Chicago, Illinois

December 2004 
(c) Copyright by

\section{MOHAMMAD M. ALSHARO'A}

December 2004 


\section{ACKNOWLEDGMENT}

I would like to thank my advisor, Michael Gosz, Associate Professor of Mechanical and Materials Engineering, for his outstanding advising and brilliant FEA teaching throughout my academic program. I owe a dept of gratitude to my coadvisor, Sudhakar Nair, Professor of Mechanical and Aerospace Engineering, for his remarkable mechanics teaching and creative $R \& D$ solutions. This work would never have existed without the support and clever ideas of my co-advisor, Daniel Kaplan, Professor of Physics. I can't find words to thank my co-advisor, Alfred Moretti, who helped me continuously in the advanced RF engineering field. Special thank goes to Kevin Cassel, Associate Professor of Mechanical and Aerospace Engineering, for his valuable guidance. Special thank also goes to Rolland Johnson, president of Muons Inc, who presented innovative gaseous RF cavities and supported me to work on many revolutionary RF structures. Special thanks to the muon collaboration members for their cooperation and especially Derun Li, Tony Ladran, Jim Norem, Alan Bross, and Yagmur Torun. Finally, I would like to acknowledge my family and friends for their encouragement. 
TABLE OF CONTENTS

Page

ACKNOWLEDGEMENT . . . . . . . . . . . . . . . iii

LIST OF TABLES . . . . . . . . . . . . . . . . . . . v vi

LIST OF FIGURES . . . . . . . . . . . . . . . . . . . . .

LIST OF SYMBOLS . . . . . . . . . . . . . . . . . . xi

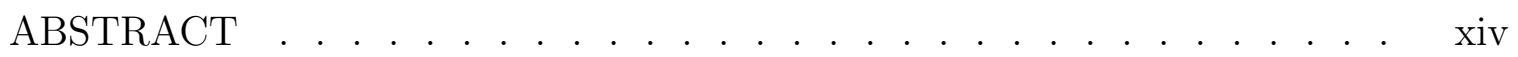

CHAPTER

1. INTRODUCTION . . . . . . . . . . . . . . . . . 1

1.1. Background and Objectives . . . . . . . . . . . . 1

1.2. Literature Review . . . . . . . . . . . . . . . 4

2. DESIGN METHODOLOGY OF RF CAVITY WINDOWS . . . 8

2.1. Description of the Test Cavity . . . . . . . . . . 8

2.2. Design Requirements . . . . . . . . . . . . . . . . 8

2.3. Design Scheme . . . . . . . . . . . . . . . . . . . 11

3. ELECTROMAGNETIC AND THERMAL ANALYSIS OF A BENCHMARK RF CAVITY MODEL . . . . . . . . . . . . 14

3.1. Problem Description and Mathematical Formulation . . . . 14

3.2. Analytical Solutions . . . . . . . . . . . . . . . . . . . 18

3.3. Finite Element Solutions . . . . . . . . . . . . . . . . 23

4. FINITE ELEMENT ANALYSIS OF THE TEST CAVITY WITH BERYLLIUM WINDOWS . . . . . . . . . . . . 31

4.1. Electromagnetic Analysis . . . . . . . . . . . . . . . . 31

4.2. Thermal Analysis . . . . . . . . . . . . . . . . 35

5. FINITE ELEMENT ANALYSIS OF THE TEST CAVITY WITH GRIDDED-TUBE WINDOWS . . . . . . . . . . . . . 38

5.1. Electromagnetic Analysis . . . . . . . . . . . . . . . . . 38

5.2. Thermal Analysis . . . . . . . . . . . . . . . . . 45 
5.3. Structural Analysis . . . . . . . . . . . . . . . . . . . 52

6. EXPERIMENTAL AND FINITE ELEMENT STUDIES OF THE PERFORMANCE OF THE TEST CAVITY . . . . . . . . . . 60

6.1. Deflection of the RF Cavity Beryllium Windows . . . . . . 60

6.2. Electromagnetic Analysis of the Cavity under RF Power Operation .................. . 63

7. CONCLUSIONS AND RECOMMENDATIONS . . . . . . . 66

7.1. Conclusions . . . . . . . . . . . . . . . . 66

7.2. Recommendations for Future Work . . . . . . . . . . . . 69

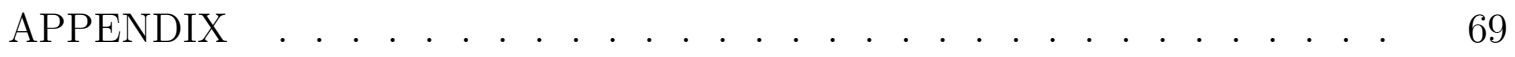

A. DESCRIPTION OF THE DAMAGE IN THE TITANIUM WINDOW DUE TO FIELD EMISSION . . . . . . . . . . . 70

B. FINITE ELEMENT FORMULATION OF THE ELECTRIC FIELD INSIDE THREE DIMENSIONAL CAVITIES . . . . . . . . . . 72

BIBLIOGRAPHY . . . . . . . . . . . . . . . . . . . . . . . . 76 


\section{LIST OF TABLES}

Table

Page

2.1 Design Parameters of the $805 \mathrm{MHz}$ Test cavity. . . . . . . . . . . 12

2.2 Material Properties of the $805 \mathrm{MHz}$ Test Cavity Windows at Room Temperature $\left(23^{\circ} \mathrm{C}\right)$. . . . . . . . . . . . . . . . . . . . . . . . 12

3.1 Design Parameters of the Cylindrical Pillbox Cavity. . . . . . . . . 16

3.2 Comparison Between the Finite Element and the Analytical Results for the Cylindrical Pillbox Cavity. . . . . . . . . . . . . . . . . . 30

4.1 Finite Element Results for the $805 \mathrm{MHz}$ Test Cavity with Beryllium Windows. .....................

5.1 Effects of Both Grid Pattern and Tube Outer Diameter on the Surface Electric Field Enhancement, . . . . . . . . . . . . . . . . . . . 44

5.2 Geometrical Parameters of the Baseline Gridded-Tube Window. . .

5.3 Finite Element Results for the $805 \mathrm{MHz}$ Test Cavity with the Baseline Gridded-Tube Window. . . . . . . . . . . . . . . . . . .

5.4 Analytical and Finite Element Solutions of the Fundamental Natural Frequency of Various RF Cavity Windows. . . . . . . . . . . . . 


\section{LIST OF FIGURES}

Figure

Page

1.1 The Test Cavity Closed by a Gridded-Tube Window and a Circular Foil (One-Eighth Section). . . . . . . . . . . . . . . . . . . . .

1.2 Layout of the International Muon Ionization and Cooling Experiment (MICE) [Alsharo'a et al. (2003)]. . . . . . . . . . . . . . 5

2.1 Cross-Sectional View of the $805 \mathrm{MHz}$ Test Cavity. . . . . . . . . 9

2.2 Iterative Design Scheme of RF Cavity Windows. . . . . . . . . . 13

3.1 The Cylindrical Pillbox Cavity: The Side View (Right) Shows the Beryllium Foils (I), Copper Window Frames (II), and the Copper Cavity Wall (III). The End View (Left) Shows the Azimuthal Direction. . . . . . . . . . . . . . . . . .

3.2 Electromagnetic Volume Mesh of the Cylindrical Pillbox Cavity and Thermal Surface Mesh of the Cavity and the Windows. (Relative Element Size with Respect to the Inner Radius of the Cavity, $r_{e} / b$, Equals About 0.03). . . . . . . . . . . . . . . . . . . . . . . .

3.3 Vector Plot of the Electric Field in the Cylindrical Pillbox Cavity $(\mathrm{MV} / \mathrm{m})$. On-Axis Electric Field $=30 \mathrm{MV} / \mathrm{m}$. . . . . . . . . . .

3.4 Vector Plot of the Magnetic Field in the Cylindrical Pillbox Cavity $(\mathrm{MA} / \mathrm{m})$. On-Axis Electric Field $=30 \mathrm{MV} / \mathrm{m}$. . . . . . . . . . .

3.5 Contour Plot of the Power Loss Density in the Cylindrical Pillbox Cavity $\left(\mathrm{W} / \mathrm{m}^{2}\right)$. . . . . . . . . . . . . . . . . . . . . . . . .

3.6 Contour plot of the Temperature Distribution in the Cylindrical

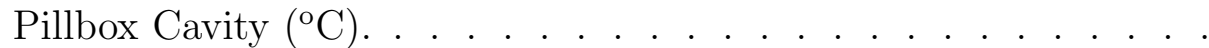

3.7 Analytical and Finite Element Electric Field Profiles in the Cylindrical Pillbox Cavity. . . . . . . . . . . . . . . . . . . . . .

3.8 Analytical and Finite Element Magnetic Field Profiles in the Cylindrical Pillbox Cavity. . . . . . . . . . . . . . . . . . . . . .

3.9 Analytical and Finite Element Power Loss Density Profiles in the Cylindrical Pillbox Cavity. . . . . . . . . . . . . . . . . . . . .

3.10 Analytical and Finite Element Temperature Profiles in the Cylindrical Pillbox Cavity. . . . . . . . . . . . . . . . . . . . . . . 
4.1 Electromagnetic Volume Mesh of the Test Cavity Closed by Beryllium Windows and Thermal Meshes of the Cavity and the Windows (Relative Minimum Element Size with Respect to the Inner Radius of the Cavity, $r_{e} / b$, Equals About 0.003). . . . . . . . . . . . . .

4.2 Contour Plot of the Electric Field Distribution in the Test Cavity Closed by Beryllium Windows $(\mathrm{MV} / \mathrm{m})$. On-Axis Electric Field = $30 \mathrm{MV} / \mathrm{m}$. . . . . . . . . . . . . . . . . . . . . . .

4.3 Contour Plot of the Magnetic Field Distribution in the Test Cavity Closed by Beryllium Windows $(\mathrm{MA} / \mathrm{m})$. On-Axis Electric Field = $30 \mathrm{MV} / \mathrm{m}$. . . . . . . . . . . . . . . . . . . . . . . . . . .

4.4 Contour Plot of the Power Loss Density in the Test Cavity Closed By Beryllium Windows $\left(\mathrm{W} / \mathrm{m}^{2}\right)$. . . . . . . . . . . . . . .

4.5 Contour Plot of the Temperature Distribution in the Beryllium Window $\left({ }^{\circ} \mathrm{C}\right)$. Edge of the Window is Cooled to a Reference Tempera-

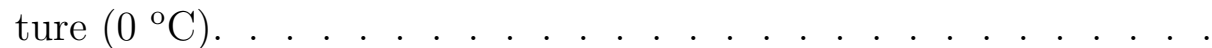

4.6 Contour Plot of the Temperature Distribution in the Test Cavity Closed by Beryllium Windows $\left({ }^{\circ} \mathrm{C}\right)$. Outer Boundary of the cavity is Cooled to a Reference Temperature $\left(0^{\circ} \mathrm{C}\right)$. . . . . . . . . . .

5.1 The Test Cavity Closed by a Grid of Non-Touching Tubes. Tube

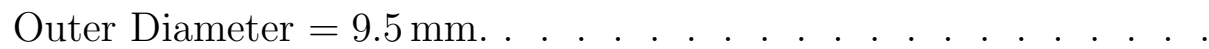

5.2 Contour Plot of the Electric Field Distribution in the Test Cavity Closed by a Grid of Non-Touching Tubes. Tube Outer Diameter $=9.5 \mathrm{~mm}(\mathrm{MV} / \mathrm{m})$. On-Axis Electric Field $=30 \mathrm{MV} / \mathrm{m}$. . . . . .

5.3 The Test Cavity Closed by a Grid of Touching Tubes. Tube Outer

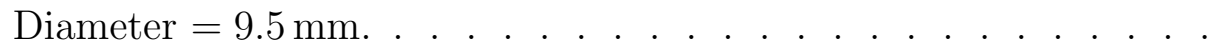

5.4 Contour Plot of the Electric Field Distribution in the Test Cavity Closed by a Grid of Touching Tubes. Tube Outer Diameter $=9.5 \mathrm{~mm}(\mathrm{MV} / \mathrm{m})$. On-Axis Electric Field $=30 \mathrm{MV} / \mathrm{m}$. . . . . .

5.5 Electromagnetic Volume Mesh of Test Cavity Closed by the Baseline Gridded-Tube Window and Thermal Surface Mesh of the GriddedTube Window (Relative Minimum Element Size with Respect to the Inner Radius of the Cavity, $r_{e} / b$, Equals About 0.002). . . . . . .

5.6 Contour Plot of the Electric Field Distribution in the Test Cavity Closed by the Baseline Gridded-Tube Window $(\mathrm{MV} / \mathrm{m})$. On-Axis Electric Field $=30 \mathrm{MV} / \mathrm{m}$. . . . . . . . . . . . . . . . . . . 
5.7 Contour Plot of the Power Loss Density in the Baseline GriddedTube Window $\left(\mathrm{W} / \mathrm{m}^{2}\right)$ (External Side). . . . . . . . . . . . . .

5.8 Contour Plot of the Power Loss Density in the Baseline GriddedTube Window $\left(\mathrm{W} / \mathrm{m}^{2}\right)$ (Internal Side). . . . . . . . . . . . . .

5.9 Contour Plot of the Temperature Distribution in the Non-Cooled Baseline Gridded-Tube Window $\left({ }^{\circ} \mathrm{C}\right)$. Grid Wall Thickness $=254 \mu \mathrm{m}$. (External Side). . . . . . . . . . . . . . . . . . . . . . . . . .

5.10 Contour Plot of the Temperature Distribution in the Non-Cooled Baseline Gridded-Tube Window $\left({ }^{\circ} \mathrm{C}\right)$. Grid Wall Thickness $=254 \mu \mathrm{m}$. (Internal Side). . . . . . . . . . . . . . . . . . . .

5.11 The Transient Maximum Temperature Profile of the Non-Cooled Baseline Gridded-Tube Window. Grid Wall Thickness $=254 \mu \mathrm{m}$. .

5.12 Dependence of the Temperature Profile on the Grid Wall Thickness for the Non-Cooled Baseline Gridded-Tube Window. . . . . . . .

5.13 Contour Plot of the Temperature Distribution in the Gas-Cooled Baseline Gridded-Tube Window $\left({ }^{\circ} \mathrm{C}\right)$. Grid Wall Thickness $=254 \mu \mathrm{m}$. (External Side). . . . . . . . . . . . . . . . . . . . . . . . .

5.14 Contour plot of the Temperature Distribution in the Gas-Cooled Baseline Gridded-Tube Window $\left({ }^{\circ} \mathrm{C}\right)$. Grid Wall Thickness $=254 \mu \mathrm{m}$. (Internal Side). . . . . . . . . . . . . . . . . . . . . .

5.15 Contour Plot of the Temperature Distribution in the Gas-Cooled Baseline Gridded-Tube Window $\left({ }^{\circ} \mathrm{C}\right)$. Grid Wall Thickness $=100 \mu \mathrm{m}$. (External Side). . . . . . . . . . . . . . . . . . . . . . .

5.16 Contour Plot of the Temperature Distribution in the Gas-Cooled Baseline Gridded-Tube Window $\left({ }^{\circ} \mathrm{C}\right)$. Grid Wall Thickness $=100 \mu \mathrm{m}$. (Internal Side). . . . . . . . . . . . . . . . . . . . . . . . . .

5.17 Dependence of the Temperature Profile on the Grid Wall Thickness for the Gas-Cooled Baseline Gridded-Tube Window. $(h=$ $\left.250 \mathrm{~W} / \mathrm{m}^{2} \mathrm{~K}\right)$. . . . . . . . . . . . . . . . . . . . . . . . . .

5.18 Finite Element Structural Mesh of the Baseline Gridded-Tube Window (Relative Minimum Element Size with Respect to the Inner Radius of the Cavity, $r_{e} / b$, Equals About 0.002). . . . . . . . . .

5.19 Dependence of the Numerical Value of the Thermal Stress on the Number of Elements for the Baseline Gridded-Tube Window. Grid Wall Thickness $=254 \mu \mathrm{m}$ (Uniform Meshing). . . . . . . . . . . 
5.20 Contour Plot of the Total Displacement of the Non-Cooled Baseline Gridded-Tube Window, $(m)$, Grid Wall Thickness $=254 \mu \mathrm{m}$ (Internal Side). . . . . . . . . . . . . . . . . . . . .

5.21 Contour Plot of the von Mises Stress in the Non-Cooled Baseline Gridded-Tube Window (Pa). Grid Wall Thickness $=254 \mu \mathrm{m}$. . . .

5.22 Contour Plot of the Total Displacement of the Gas-Cooled Baseline Gridded-Tube Window (m). Grid Wall Thickness $=254 \mu \mathrm{m}$ (External Side). . . . . . . . . . . . . . . . . . . . . . . .

5.23 Contour Plot of the Total Displacement of the Gas-Cooled Baseline Gridded-Tube Window (m). Grid Wall Thickness $=254 \mu \mathrm{m}$ (Internal Side). . . . . . . . . . . . . . . . . . . . . . . .

5.24 Contour Plot of the von Mises Stress in the Gas-Cooled Baseline Gridded-Tube Window (m). Grid Wall Thickness $=254 \mu \mathrm{m}$ (External Side). . . . . . . . . . . . . . . . . . . . . . . . .

5.25 Contour Plot of the von Mises Stress in the Gas-Cooled Baseline Gridded-Tube Window (Pa). Grid Wall Thickness $=254 \mu \mathrm{m}$ (Internal Side). . . . . . . . . . . . . . . . . . . . . . . . . .

5.26 Mode Shape Associated With the Fundamental Natural Frequency of the Baseline Gridded-Tube Window. Grid Wall Thickness = $254 \mu \mathrm{m}$. . . . . . . . . . . . . . . . . . . . . . . .

6.1 Finite Element and Experimental Results of the Out-Of-Plane Deflection of the Stress Free Aluminum Window . . . . . . . . .

6.2 Finite Element and Experimental Results of the Out-Of-Plane Deflection of the Pre-Stressed Beryllium Window . . . . . . . . . .

A.1 Damage in the Titanium Vacuum Window Caused by Field Emission [Norem at al. (2003c)]. . . . . . . . . . . . . . . . . . . .

A.2 Scanning Electron Microscope Image of Copper Splattered on the Inner Surface of the Titanium Window Caused by Field Emission ([Norem at al. $(2003 \mathrm{c})]$. . . . . . . . . . . . . . . . . . . . . 71

B.1 Tetrahedral Element with Four Nodes and Six Edges. . . . . . . 74 


\section{LIST OF SYMBOLS}

Symbol

Definition

$A_{c} \quad$ Cross Sectional Area Normal to Heat Flow Direction $\left(\mathrm{m}^{2}\right)$

$A_{s} \quad$ Surface Area Subjected to Power Loss Density $\left(\mathrm{m}^{2}\right)$

$\bar{a} \quad$ Plate Radius (m)

$\vec{B} \quad$ Magnetic Flux Density (T)

b Cavity Inner Radius (m)

$c_{1}, . ., c_{4} \quad$ Integration Constants

d Length of the Cavity

E Modulus of Elasticity of the Material (Pa)

$\vec{E} \quad$ Electric Field $(\mathrm{V} / \mathrm{m})$

$E_{0} \quad$ On-axis Electric Field $(\mathrm{V} / \mathrm{m})$

$E_{m} \quad$ Electric Field at the Tip of the Emitter $(\mathrm{V} / \mathrm{m})$

$E_{s} \quad$ Average Surface Electric Field $(\mathrm{V} / \mathrm{m})$

F Duty Factor

$F_{0} \quad$ Fundamental Natural Frequency $(\mathrm{Hz})$

$\vec{H} \quad$ Magnetic Field $(\mathrm{A} / \mathrm{m})$

$i \quad$ Field Emission Current Density $\left(\mathrm{A} / \mathrm{m}^{2}\right)$

$k \quad$ Thermal Conductivity $(\mathrm{W} / \mathrm{m} \cdot \mathrm{K})$

$m \quad$ Mass of the Plate $(\mathrm{kg})$

$\vec{n} \quad$ Unit Outward Normal to the Boundary.

P $\quad$ Power Loss (W)

$P_{t} \quad$ Total Power Loss (W) 


\begin{tabular}{|c|c|}
\hline$Q_{f}$ & Quality Factor \\
\hline$Q_{r}$ & Radial Heat Flow (W) \\
\hline$q$ & Power Loss Density $\left(\mathrm{W} / \mathrm{m}^{2}\right)$ \\
\hline$R_{c}$ & Conductor Surface Resistance $(\Omega)$ \\
\hline$r$ & Radial Direction \\
\hline$T$ & Temperature $\left({ }^{\circ} \mathrm{C}\right)$ \\
\hline$t$ & Foil Thickness or Grid Wall Thickness (m) \\
\hline$U_{t}$ & Total Stored Energy (J) \\
\hline$V$ & Volume $\left(\mathrm{m}^{3}\right)$ \\
\hline$z$ & Axial Coordinate \\
\hline$\beta$ & $\begin{array}{l}\text { Ratio of the Electric Field at the Tip of the Emitter to the } \\
\text { Average Surface Electric Field }\end{array}$ \\
\hline$\beta_{t o t}$ & Total Electric Field Enhancement \\
\hline$\epsilon_{0}$ & Permittivity of Vacuum $(\mathrm{F} / \mathrm{m})$ \\
\hline$\gamma$ & $\begin{array}{l}\text { First Root of Zeroth-Order Bessel Function Divided by the } \\
\text { Inner Radius of the Cavity, }(\gamma \approx 2.405 / \mathrm{b})\end{array}$ \\
\hline$\mu_{0}$ & Magnetic Permeability of Vacuum $(\mathrm{H} / \mathrm{m})$ \\
\hline$\mu_{c}$ & Magnetic Permeability of the Conductor $(\mathrm{H} / \mathrm{m})$ \\
\hline$\nu$ & Poisson's Ratio of the Material \\
\hline$\omega$ & Resonance Frequency (Hz) \\
\hline$\phi$ & Azimuthal Direction \\
\hline$\phi_{F N}$ & Work Function of the Material $(\mathrm{eV})$ \\
\hline$\sigma$ & Electrical Conductivity (mho/m) \\
\hline
\end{tabular}


$\zeta \quad$ Surface Electric Field Enhancement, $\left(\zeta=E_{s}^{\max } / E_{0}\right)$

$\Gamma \quad$ Conductor Area $\left(\mathrm{m}^{2}\right)$

I Subscript Refers to the Beryllium Window

II Subscript Refers to the Frame of the Window

III Subscript Refers to the Cylindrical Wall of the Cavity 


\begin{abstract}
Electromagnetic, thermal and structural analyses of radio-frequency (RF) cavities were performed as part of a developmental RF cavity program for muon cooling. RF cavities are necessary to provide longitudinal focusing of the muons and to compensate for their energy loss. Closing the cavity ends by electrically conducting windows reduces the power requirement and increases the on-axis electric field for a given maximum surface electric field. Many factors must be considered in the design of RF cavity windows. RF heating can cause the windows to deform in the axial direction of the cavity. The resulting thermal stresses in the window must be maintained below the yield stress of the window material. The out-of-plane deflection must be small enough so that the consequent frequency shift is tolerable. For example, for an 805 $\mathrm{MHz}$ cavity, the out-of-plane deflection must be kept below 25 microns to prevent the frequency of the cavity from shifting more than $10 \mathrm{kHz}$. In addition, the window design should yield smooth electric and magnetic fields, terminate field leakage beyond the window, and minimize beam scattering. In the present thesis, gridded-tube window designs were considered because of their high structural integrity.
\end{abstract}

As a starting point in the analysis, a cylindrical pillbox cavity was considered as a benchmark problem. Analytical and finite element solutions were obtained for the electric and magnetic fields, power loss density, and temperature profile. Excellent agreement was obtained between the analytical and finite element results. The finite element method was then used to study a variety of gridded-tube windows. It was found that cooling of the gridded-tube windows by passing helium gas inside the tubes significantly reduces the out-of-plane deflection and the thermal stresses. Certain tube geometries and grid patterns were found to satisfy all of the design requirements. 


\section{CHAPTER 1}

\section{INTRODUCTION}

In Section 1.1, Radio-Frequency (RF) cavities and RF cavity windows are described, the objectives of the present work are briefly summarized, and the thesis outline is presented. In Section 1.2, a literature review is presented.

\subsection{Background and Objectives}

Recently, a variety of fields such as telecommunications, medicine, and highenergy particle physics have expressed significant interest in RF applications. The present work demonstrates the use of the finite element method for the design of structures subjected to RF power. In particular, we consider RF cavities, which are metallic bodies that resonate in a desired mode of oscillation and at a desired frequency.

The muon is an elementary particle that is about 200 times heavier than the electron and has a very short average lifetime of about $2.2 \mu \mathrm{s}$. The production of high-energy muon beams requires muon cooling. In thermodynamics, cooling means reduction of the temperature of the material, whereas in particle physics, cooling means reduction of both the beam size and divergence. Muon cooling requires the use of RF cavities as accelerating structures [Alsharo'a et al. (2003)].

The ends of the RF cavity can be closed by electrically conducting materials called RF cavity windows to reduce the power requirement and to increase the onaxis electric field for a given maximum surface electric field [Moretti et al. (2000)]. The RF cavity window should be thin and made of material of long radiation length to reduce the beam scattering. Thin, circular foils and thin-walled, gridded-tube windows have been proposed as window candidates. The gridded-tube windows are grid-like structures composed of tubes. Figure 1.1 shows a one-eighth section of a 
geometrical model of an RF cavity closed by a circular foil from one side and a gridded-tube window from the other side. Many factors must be considered in the design of RF cavity windows. RF heating can cause the windows to deform outof-plane (in the axial direction of the cavity). The resulting thermal stresses in the window must be maintained below the yield stress of the window material. The out-of-plane deflection must be small enough so that the consequent frequency shift is tolerable. For example, for an $805 \mathrm{MHz}$ cavity, the out-of-plane deflection must be kept below 25 microns to prevent the frequency of the cavity from shifting more than $10 \mathrm{kHz}$ [Hartman et al. (2000), Hartman et al. (2001)]. In addition, the window design should yield smooth electric and magnetic fields, terminate field leakage past the window, and minimize beam scattering. These requirements will be explained later in Section 5.1. In the present thesis, gridded-tube window designs are considered because of their high structural integrity.

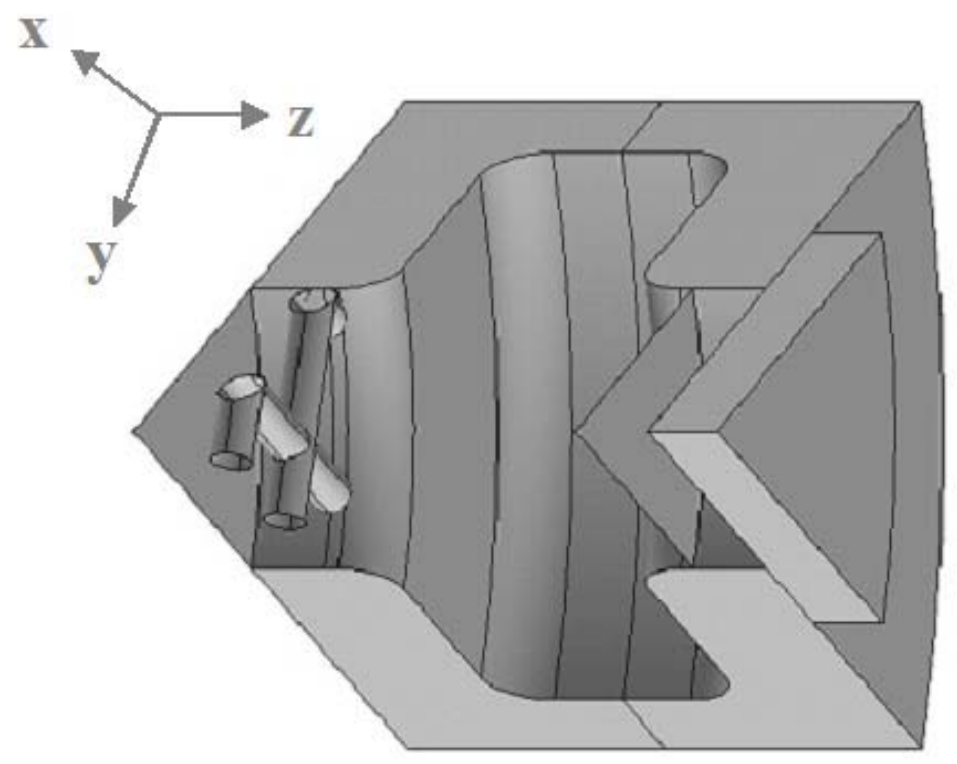

Figure 1.1. The Test Cavity Closed by a Gridded-Tube Window and a Circular Foil (One-Eighth Section). 
In the present work, a finite element method for the electromagnetic and mechanical design of RF cavity windows is presented. The method is based on performing finite element analyses from electromagnetic, thermal, and structural points of view in an iterative manner. The procedure involves several steps. First, the resonance frequency, electric and magnetic fields, quality factor, and power loss density are calculated. Next, the power loss density results are transferred from the electromagnetic mesh to a thermal mesh to calculate the temperature results. The temperature results are then transferred to a structural mesh to calculate the displacement and the stresses. The procedure will be applied to a test cavity for a variety of RF cavity window configurations. The test cavity is an $805 \mathrm{MHz}$ pillbox cavity used to test the concept of pillbox cavities closed by RF windows. The RF cavity window configurations include thin, circular foils and thin-walled, gridded-tube patterns.

To outline the remainder of the thesis, in the next section, we present a literature review. In Chapter 2, we present a design methodology for RF cavity windows. This chapter includes a description of the test cavity, a detailed discussion of the window design requirements, and a description of the iterative design scheme. In Chapter 3, a cylindrical $805 \mathrm{MHz}$ pillbox cavity, similar to the test cavity, is studied. The cavity is closed by beryllium windows at both ends. Analytical and finite element solutions for the electric field, magnetic field, stored energy, quality factor, total power loss, power loss density, and temperature distribution are obtained for that cavity. Excellent agreement is obtained between the analytical and the finite element results.

Having validated the finite element method with analytical results, we present finite element results for the test cavity closed by beryllium windows in Chapter 4 . Next, in Chapter 5, finite element analyses are carried out for the same test cavity for a variety of gridded-tube arrangements. In Chapter 6, experimental and numerical 
studies of the performance of the test cavity are presented. In Chapter 7, concluding remarks and recommendations for future work are presented. Finally, figures that illustrate the damage in the titanium window due to field emission are presented in Appendix A, and the finite element formulation used to obtain the electric field inside the RF cavity is presented in Appendix B.

\subsection{Literature Review}

Muon ionization cooling requires passing the muon beam through a channel containing energy-absorbing material and accelerating structures [Alsharo'a et al. (2003) and Kaplan (2001)]. The beam loses both transverse and longitudinal momentum by ionization energy loss while passing through the energy-absorbing material. The beam then gains longitudinal momentum by passing through accelerating structures. Consequently, a reduction in both the beam size and divergence can be achieved.

An international muon ionization and cooling experiment (MICE) has been proposed to show that ionization cooling of a muon beam is feasible [Alsharo'a et. al. (2003)]. The energy-absorbing materials (absorbers) used in the experiment are cylindrically shaped vessels that contain liquid hydrogen. The accelerating structures used in the experiment are RF cavities. Both the RF cavities and the absorbers are subjected to focusing magnetic fields. MICE is described in this section for two reasons. The first reason is to illustrate the use of RF cavities in muon ionization cooling. The second reason is to emphasize that the RF cavity window studies reported in the present thesis may aid the RF cavity design in MICE and other cooling experiments. In their study of RF induced background in MICE, [Norem et al. (2003)b] concluded that the use of large, flat, beryllium windows may be unfeasible. In the present work, a variety of gridded windows have been studied as an alternative to the beryllium windows. The layout under consideration for MICE is illustrated in Figure 1.2. 


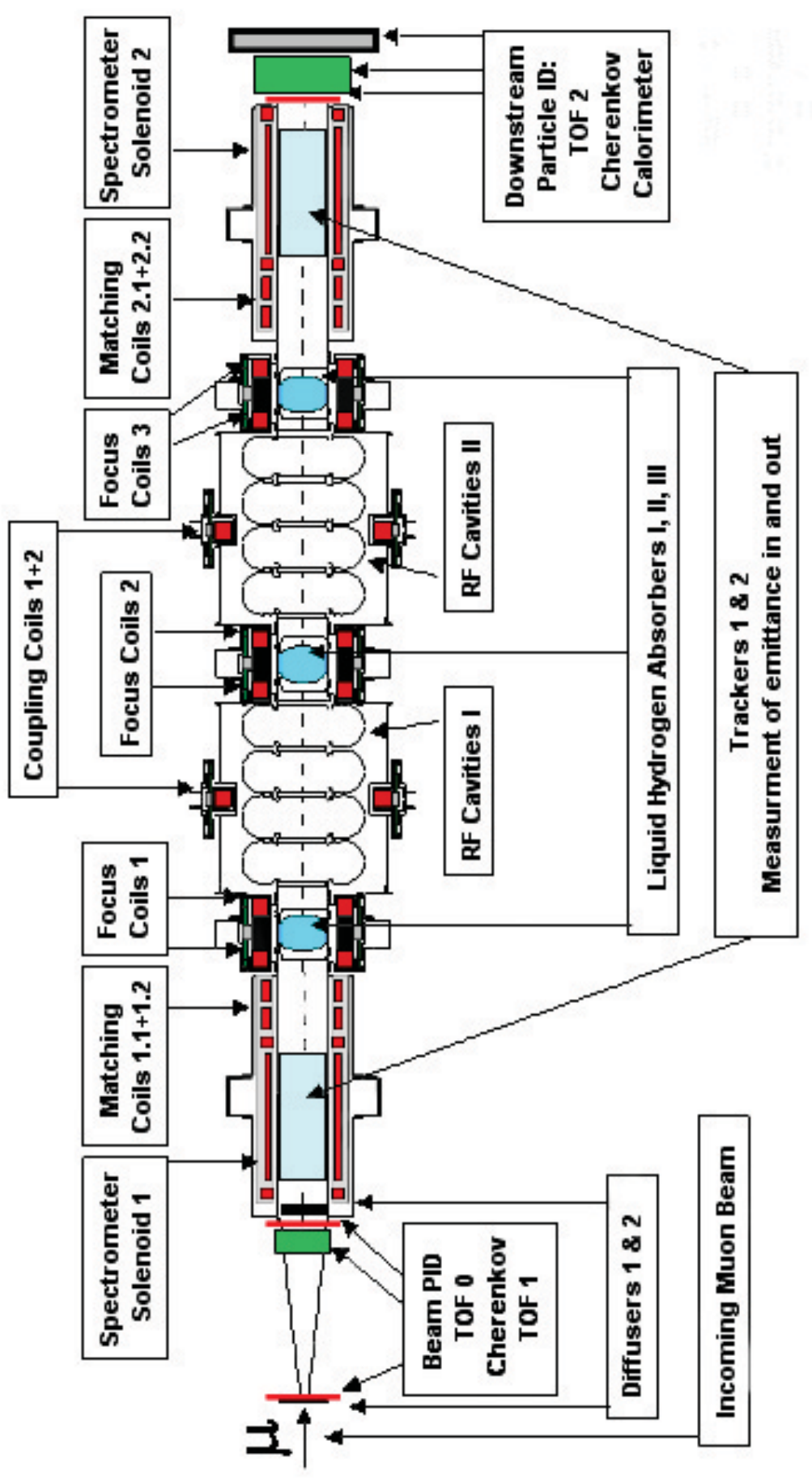

Figure 1.2. Layout of the International Muon Ionization and Cooling Experiment (MICE) [Alsharo'a et al. (2003)]. 
If a conducting surface is subjected to an intense electric field, it can emit electrons [Fowler and Nordheim (1928)]. In the case of RF cavities, these electrons can be accelerated by the RF field. This field emission must be avoided because it is a precursor to vacuum breakdown and generation of dark current. A field emission study has been performed for an open $805 \mathrm{MHz}$ cavity [Norem et al. (2003)a, Norem et al. (2003)c, and $\mathrm{Wu}(2002)]$. In that study, the ratio of the maximum electric field at the surface of the cavity to the on-axis electric field was about 2.6. The results showed that dark current was approximately proportional to the surface electric field raised to the tenth power. During the operation of the cavity at a maximum surface electric field of $54 \mathrm{MV} / \mathrm{m}$ and a solenoidal field of $2.5 \mathrm{~T}$, local heating due to dark current melted the titanium vacuum window that was placed at the end of the cavity. It was obvious that field emission limits the operation of the open $805 \mathrm{MHz}$ cavity.

RF cavity windows serve to reduce the power requirement and to increase the on-axis electric field for a given maximum surface electric field [Moretti et al. (2000) and Li et al. (2003)a]. Studies of the design of RF cavities for muon cooling purposes have been performed [Corlett et al. (1999), Rimmer et al. (2001), Li et al. (2001), and Turner et al. (1998)]. In RF cavities closed by beryllium foils, the maximum surface electric field is approximately equal to the on-axis electric field. In conventional open RF cavities, the maximum surface electric field is typically more than twice the on-axis electric field. As described earlier, the dark current was proportional to the surface field raised to a high power. Consequently, RF cavity windows cause a considerable decrease in dark current.

The temperature rise and the thermal stresses in the window due to RF heating are of major concern. Deflection analyses of flat, circular, beryllium foils for $805 \mathrm{MHz}$ cavity windows subjected to various thermal loads have been carried out numerically and experimentally [Hartman et al. (2000), Hartman et al. (2001), Li et al.(2002)b, 
and Li et al. (2003)b]. Several window designs were analyzed, including non-stressed aluminum flat foils, pre-stressed beryllium flat foils, stepped-thickness beryllium foils, and pre-curved beryllium windows. Window designs for $201.25 \mathrm{MHz}$ cavities were studied by [Moretti et al. (2000), Li et al. (2003)a]. Results indicated that flat foils may be inappropriate for the 201.25 MHz cavities because of their large out-of-plane displacement. In the present thesis, an $805 \mathrm{MHz}$ test cavity, which is smaller and more practical for research and development purposes than the $201 \mathrm{MHz}$ cavity, is considered.

The gridded-tube RF cavity windows studied in the present thesis are new designs that have not been previously considered. The present work shows that gridded-tube windows are feasible for use in RF cavity applications provided that the grid pattern, tube outer diameter, tube wall thickness, and other design parameters are chosen appropriately. 


\section{CHAPTER 2 \\ DESIGN METHODOLOGY OF RF CAVITY WINDOWS}

In this Chapter, the test cavity is described, electromagnetic, thermal, and structural design requirements for RF cavity windows are explained, and a design scheme for RF cavity windows is presented.

\subsection{Description of the Test Cavity}

The test cavity shown in Figure 2.1 is used for testing to understand the performance of RF cavities in the muon-cooling channel. The cavity is made of copper and closed by windows at both ends. The cavity receives RF power from a coupler through a small hole in the upper right side of the cavity wall. A titanium vacuum window covers the cavity from the left side, and a stainless steel plate covers the cavity from the right side. The cavity design parameters including resonance frequency, onaxis electric field, quality factor, total power loss, and RF cavity window dimensions are given in Table 2.1.

\subsection{Design Requirements}

The design requirements for the RF cavity windows are classified into material, electromagnetic, and mechanical requirements. A successful window design should satisfy all three requirements.

\subsubsection{Material Requirements..}

There are several requirements for the window material. The window should be made of a material of long radiation length to reduce the scattering of the beam as it passes through the window. Also, the electrical conductivity of the window material should be high to decrease the power loss (the power dissipated in the window is inversely proportional to the square root of the electrical conductivity of the window 


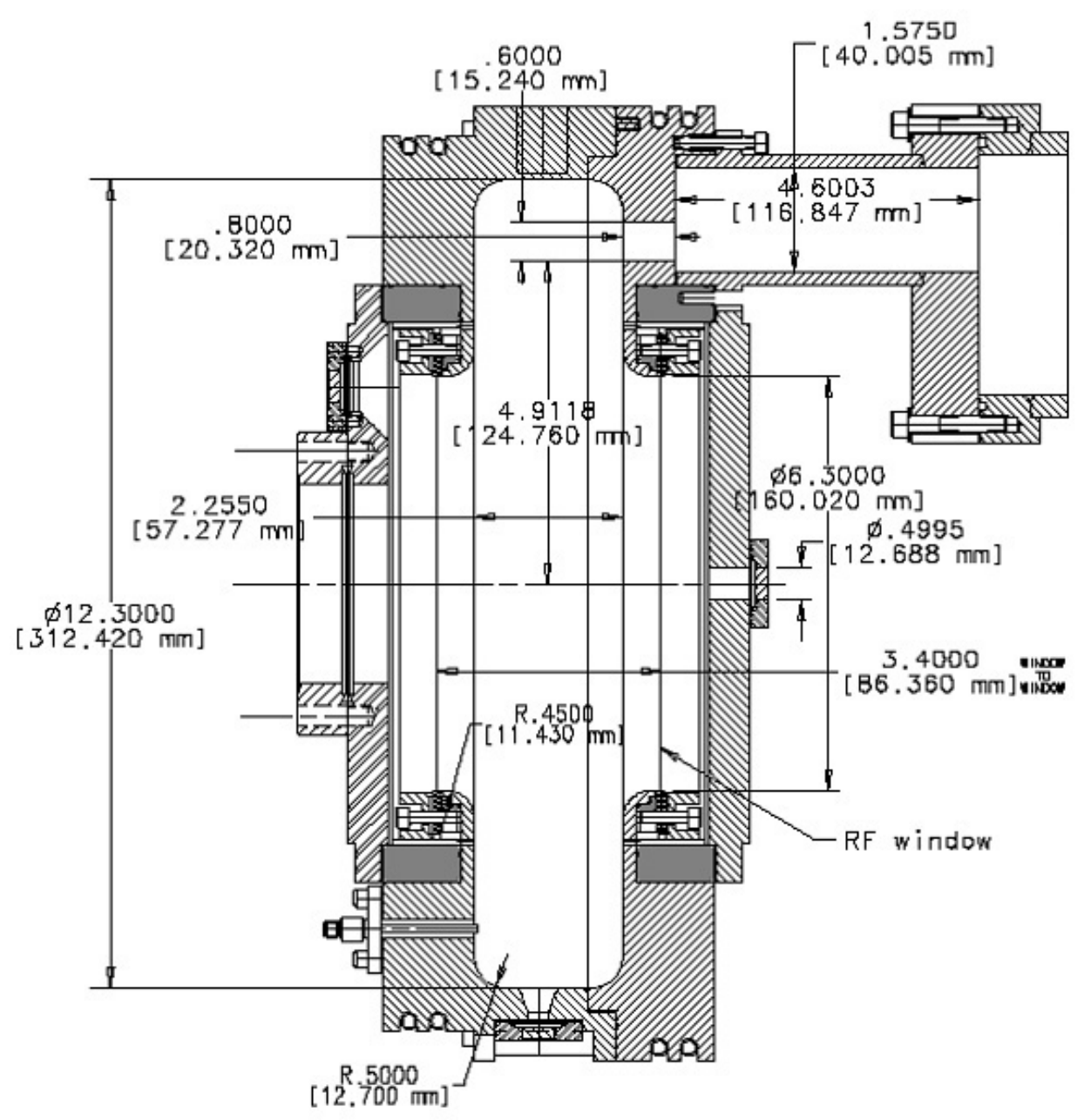

Figure 2.1. Cross-Sectional View of the $805 \mathrm{MHz}$ Test Cavity. 
material). In addition, the thermal conductivity should be high so as to decrease the temperature rise in the window, and consequently decrease the out-of-plane deflection and the thermal stresses. Finally, the material should have a low thermal expansion coefficient to reduce the out-of plane deflection. These requirements suggest that high-purity beryllium and some aluminum alloys are acceptable materials for the RF cavity windows. In the present work, beryllium (Be IF1) windows and gridded-tube aluminum (Al 6061-T6) windows are considered. Design-related material properties are listed in Table 2.2.

\subsubsection{Electromagnetic Requirements.}

The design of the window should produce a low surface electric field enhancement. The surface electric field enhancement is defined as the ratio of the maximum surface electric field to the on-axis electric field. Reducing the surface electric field enhancement serves to reduce the field emission effects. Also, the design of the window should result in a smooth electric field distribution. Field distortion can increase the spread of the beam energy and degrade the cooling process. Finally, the window should be designed to terminate the electric field (i.e., cause negligible electric field leakage to the regions beyond the window). This is because the closed-cell RF cavities should operate independently of each other. Electric field leakage to a cavity will affect the electric field profile in that cavity and consequently affect the operation of that cavity. For an RF cavity closed by beryllium windows, the electric field is terminated at the windows. For an RF cavity closed by gridded-tube windows, the design of the grid should minimize electric field leakage through the spaces in the grid. These requirements will be studied later in Section 5.1 for a variety of gridded-tube patterns. 


\subsubsection{Mechanical Requirements.}

RF heating can cause the windows to deform in the axial direction of the cavity. The out-of-plane deflection must be small enough so that the consequent frequency shift is tolerable. For example, for an $805 \mathrm{MHz}$ cavity, the out-of-plane deflection of the window must be kept below 25 microns to prevent the frequency of the cavity from shifting more than $10 \mathrm{kHz}$ [Hartman et al. (2000), Hartman et al. (2001)]. Also, the resulting thermal stresses in the window must be maintained below the yield stress. In addition, the thickness of the window should be small in order to decrease the scattering of the beam as it passes through the window.

\subsection{Design Scheme}

The objective is to select the optimal combination of window design parameters so that the design requirements are satisfied. The design parameters of the beryllium foils are the dimensions of the foil (foil diameter and foil thickness). For the griddedtube windows, the design parameters include tube outer diameter, tube wall thickness, number of tubes per grid, type of coolant, coolant flow rate, and gridded-tube pattern (touching arrays of tubes, non-touching arrays of tubes, or fully overlapped arrays of tubes).

The steps used for designing RF cavity windows in the present thesis are shown in Figure 2.2. First, values for the design parameters are chosen. Then, electromagnetic, thermal and structural finite element analyses are performed. In the electromagnetic analysis, the electric and magnetic fields, the resonance frequency, and the quality factor are calculated. In the thermal analysis, the total power loss, power loss density, and temperature profile are obtained. In the structural analysis, the displacement and the thermal stresses as a result of the temperature increase are obtained. This procedure is repeated until all of the design requirements are satisfied. 
Table 2.1. Design Parameters of the $805 \mathrm{MHz}$ Test cavity.

\begin{tabular}{cc}
\hline \hline Parameter & Value \\
\hline Frequency $(\mathrm{MHz})$ & 805.0 \\
On-axis electric field $(\mathrm{MV} / \mathrm{m})$ & 30.0 \\
Quality factor & 18,800 \\
RF repetition rate $(\mathrm{Hz})$ & 5.0 \\
RF pulse length $(\mu \mathrm{s})$ & 50.0 \\
Shunt impedance $(\mathrm{M} \Omega / \mathrm{m})$ & 38.0 \\
Duty factor $(\%)$ & 0.025 \\
Total power loss $(\mathrm{W})$ & 500.0 \\
Cavity inner radius $(\mathrm{cm})$ & 15.62 \\
Cavity length $(\mathrm{cm})$ & 8.64 \\
Beryllium window diameter $(\mathrm{cm})$ & 16.01 \\
Gridded-tube window diameter $(\mathrm{cm})$ & 16.01 \\
Beryllium window thickness $(\mu \mathrm{m})$ & 127.0 \\
\hline
\end{tabular}

Table 2.2. Material Properties of the $805 \mathrm{MHz}$ Test Cavity Windows at Room Temperature $\left(23^{\circ} \mathrm{C}\right)$.

\begin{tabular}{ccc}
\hline \hline Property & Aluminum 6061-T6 & Beryllium IF1 \\
\hline Electrical conductivity $(\mathrm{mho} / \mathrm{m})$ & $0.25 \times 10^{8}$ & $0.23 \times 10^{8}$ \\
Thermal conductivity $(\mathrm{W} / \mathrm{m} \cdot \mathrm{K})$ & 166.9 & 216.0 \\
Radiation length $(\mathrm{cm})$ & 8.90 & 35.17 \\
\hline
\end{tabular}




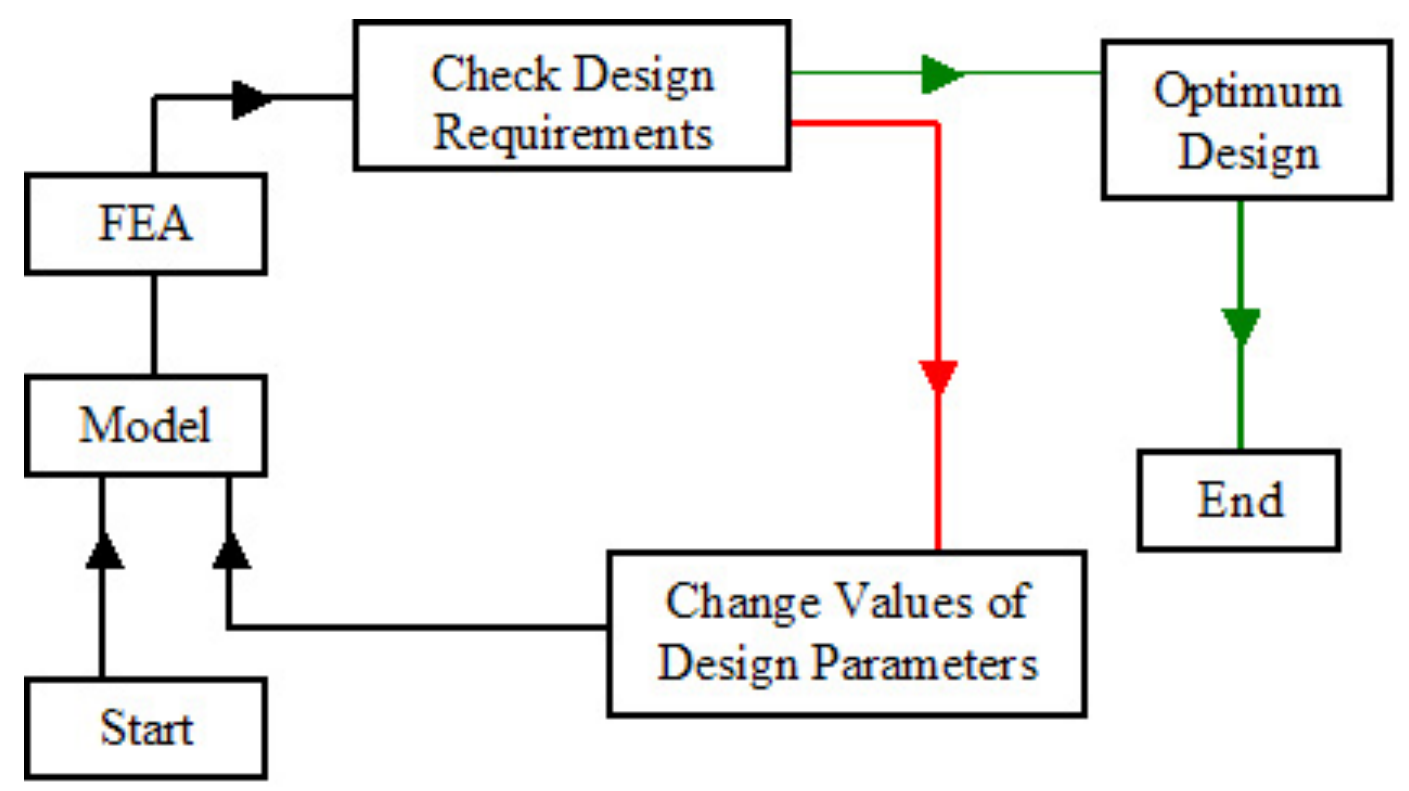

Figure 2.2. Iterative Design Scheme of RF Cavity Windows. 


\section{CHAPTER 3 \\ ELECTROMAGNETIC AND THERMAL ANALYSIS OF A BENCHMARK RF CAVITY MODEL}

In this Chapter, electromagnetic and thermal studies of a cylindrical pillbox cavity are discussed. In Section 3.1, the problem description and mathematical formulation are presented. In Section 3.2, analytical solutions for the frequency, electromagnetic fields, quality factor, power loss density and temperature are obtained. In Section 3.3, finite element solutions for the quantities mentioned above are obtained. Finally, comparisons between the analytical and finite element solutions are presented.

\subsection{Problem Description and Mathematical Formulation}

The benchmark cavity is a circular, cylindrical pillbox cavity closed by circular, flat, beryllium foils at both ends as shown in Figure 3.1. This cavity is called benchmark because it will be studied as a sample problem to verify the finite element procedure that will be applied later to the test cavity. The main geometrical difference between the benchmark cavity and the test cavity is that the test cavity has area fillets at all edges, whereas the benchmark cavity has sharp corners. The design parameters of the benchmark cavity including the on-axis electric field, dimensions, and material properties are given in Table 3.1. 

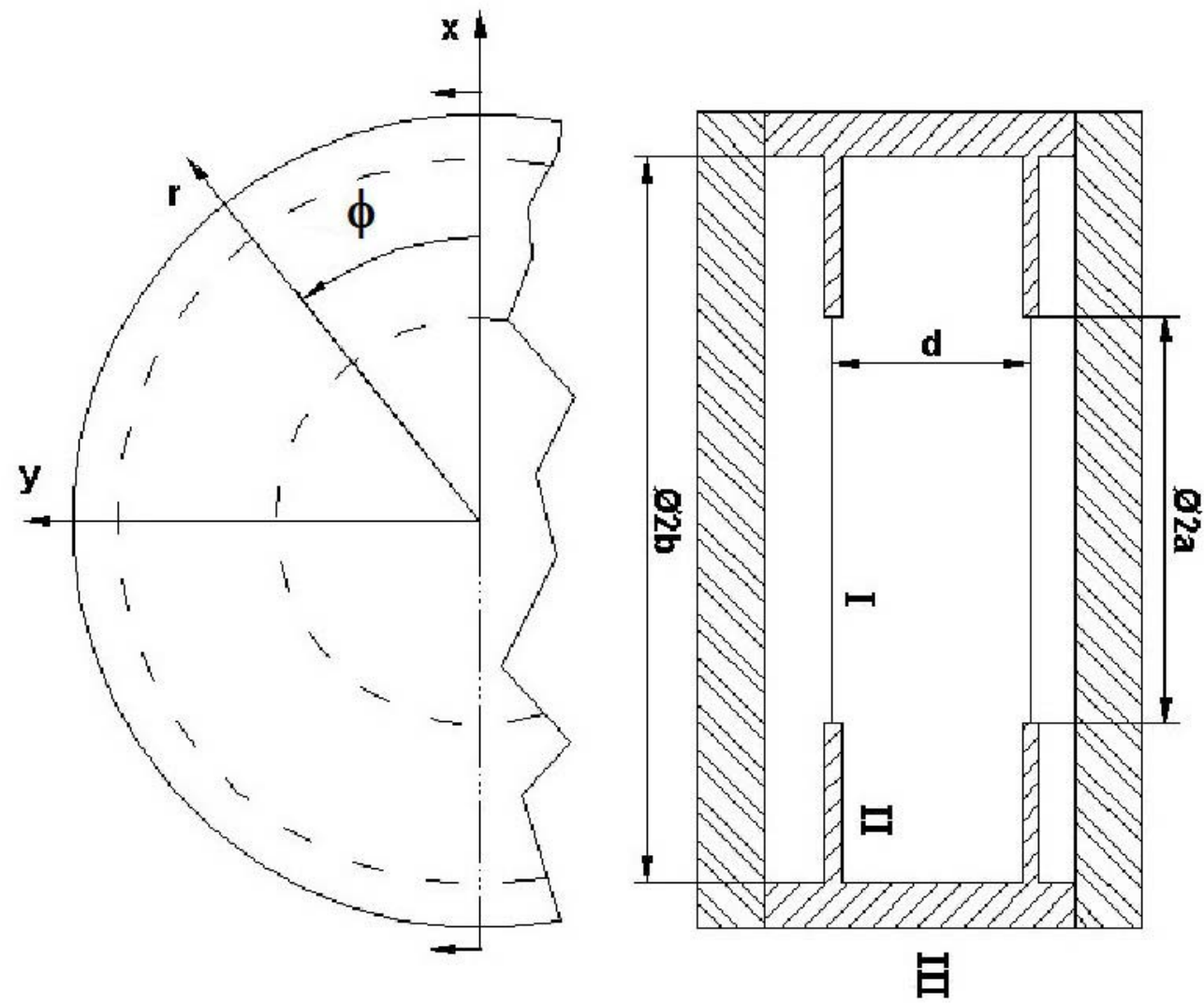

Figure 3.1. The Cylindrical Pillbox Cavity: The Side View (Right) Shows the Beryllium Foils (I), Copper Window Frames (II), and the Copper Cavity Wall (III). The End View (Left) Shows the Azimuthal Direction. 
Table 3.1. Design Parameters of the Cylindrical Pillbox Cavity.

\begin{tabular}{cc}
\hline \hline Parameter & Value \\
\hline On-axis electric field $(\mathrm{MV} / \mathrm{m})$ & 30.0 \\
Duty factor $(\%)$ & 0.025 \\
Cavity inner radius $(\mathrm{mm})$ & 142.55 \\
Cavity length $(\mathrm{mm})$ & 77.94 \\
Beryllium window diameter $(\mathrm{mm})$ & 160.01 \\
Beryllium window thickness $(\mathrm{mm})$ & 0.127 \\
Window frame thickness $(\mathrm{mm})$ & 4.0 \\
Window electrical conductivity $(\mathrm{mho} / \mathrm{m})$ & $0.23 \times 10^{8}$ \\
Frame electrical conductivity $(\mathrm{mho} / \mathrm{m})$ & $0.59 \times 10^{8}$ \\
Cavity wall electrical conductivity $(\mathrm{mho} / \mathrm{m})$ & $0.59 \times 10^{8}$ \\
Window thermal conductivity $(\mathrm{W} / \mathrm{m} \cdot \mathrm{K})$ & 216.0 \\
Frame thermal conductivity $(\mathrm{W} / \mathrm{m} \cdot \mathrm{K})$ & 385.0 \\
Cavity wall thermal conductivity $(\mathrm{W} / \mathrm{m} \cdot \mathrm{K})$ & 385.0 \\
\hline
\end{tabular}

The fields in the neighborhood of a good, but not perfect, conductor must behave similarly as those for a perfect conductor [Jackson (1998)]. Therefore, since the windows and the cavity are good conductors, to compute the fields, we assume that the conductors are perfect. Assuming that the space contained inside the cavity is a vacuum and the fields are sinusoidally time dependent, the three-dimensional, Maxwell equations take the form

$$
\begin{aligned}
& \vec{\nabla} \times \vec{E}=\mathrm{i} \omega \vec{B} \\
& \vec{\nabla} \cdot \vec{B}=0 \\
& \vec{\nabla} \times \vec{B}=-\mathrm{i} \mu_{0} \epsilon_{0} \omega \vec{E} \\
& \vec{\nabla} \cdot \vec{E}=0,
\end{aligned}
$$

where $\vec{E}$ is the electric field, $\vec{B}$ is the magnetic flux density, $\omega$ is the frequency, $\mu_{0}$ is the magnetic permeability of vacuum, and $\epsilon_{0}$ is the permittivity of vacuum. The 
previous equations can be rewritten as

$$
\begin{aligned}
& \left(\nabla^{2}+\mu_{0} \epsilon_{0} \omega^{2}\right) \vec{E}=0 \\
& \left(\nabla^{2}+\mu_{0} \epsilon_{0} \omega^{2}\right) \vec{B}=0 .
\end{aligned}
$$

At points on the boundary (windows and cavity surfaces), the electric field is perpendicular to the surface and the magnetic field is tangential to the surface, i.e.,

$$
\begin{aligned}
& \vec{n} \times \vec{E}=0 \\
& \vec{n} \cdot \vec{B}=0,
\end{aligned}
$$

where $\vec{n}$ is the unit outward normal to the boundary.

The energy flow in the cavity is represented by the Poynting vector given by $\vec{S}=(\vec{E} \times \vec{H})$, where $\vec{H}$ is the magnetic field $\left(\vec{H}=\vec{B} / \mu_{0}\right)$. The Poynting vector has the dimension of power per unit area. The time-averaged power dissipated in the conductor is given by

$$
\frac{\mathrm{d} P}{\mathrm{~d} \Gamma}=\frac{R_{c}}{2}|\vec{n} \times \vec{H}|^{2}
$$

where $\Gamma$ is the conductor surface. The quantity $R_{c}$ in Equation (3.4) is the conductor surface resistance given by

$$
R_{c}=\sqrt{\frac{\omega \mu_{c}}{2 \sigma}}
$$

where $\mu_{c}$ is the magnetic permeability of the conductor and $\sigma$ is the electrical conductivity of the conductor. Integrating Equation (3.4) with respect to $\Gamma$ gives the power loss in each conductor, $P$, as

$$
P=\frac{R_{c}}{2} \int_{\Gamma}|\vec{n} \times \vec{H}|^{2} \mathrm{~d} \Gamma
$$

The cavity is subjected to intermittent RF power operation because continuous operation at maximum power would cause a high temperature rise in the windows and the cavity. The power duty factor, $F$, represents the ratio of the actual average 
power dissipated in the cavity to the maximum power. The numerical value used in the present analysis for the duty factor is reported in Table 3.1. The actual average power dissipated in the conductors, $P_{c}$, is thus given by

$$
P_{c}=F \frac{R_{c}}{2} \int_{\Gamma}|\vec{n} \times \vec{H}|^{2} \mathrm{~d} \Gamma
$$

and the power loss density function, defined as the power loss per unit area, is given by

$$
q=\frac{F R_{c}}{2}|\vec{n} \times \vec{H}|^{2}
$$

The power loss density will be used in the thermal analysis to obtain the temperature distribution in the windows and the frame.

\subsection{Analytical Solutions}

The electric and magnetic fields can be expressed as [see, e.g., Jackson (1998)]

$$
\begin{aligned}
& E(x, y, z, t)=E(x, y) e^{ \pm \mathrm{i} k z-\mathrm{i} w t} \\
& B(x, y, z, t)=B(x, y) e^{ \pm \mathrm{i} k z-\mathrm{i} w t}
\end{aligned}
$$

where $t$ is time, $k$ is the wave number (defined below), and $z$ represents the axial direction in the cavity. Substituting Equation (3.9) into Equation (3.2) gives

$$
\begin{aligned}
& \frac{\partial^{2} \vec{E}}{\partial x^{2}}+\frac{\partial^{2} \vec{E}}{\partial y^{2}}+\left(\mu_{0} \epsilon_{0} \omega^{2}-k^{2}\right) \vec{E}=0 \\
& \frac{\partial^{2} \vec{B}}{\partial x^{2}}+\frac{\partial^{2} \vec{B}}{\partial y^{2}}+\left(\mu_{0} \epsilon_{0} \omega^{2}-k^{2}\right) \vec{B}=0 .
\end{aligned}
$$

To satisfy the boundary conditions at cavity ends, $k$ is defined as

$$
k=p \pi / d
$$

where $p$ is an integer ranging from 0 to $\infty$, and $d$ is the length of the cavity illustrated in Figure 3.1. Solving for the transverse magnetic electric field gives

$$
E_{z}=E_{0} J_{m}\left(\gamma_{m n} r\right) \cos \left(\frac{p \pi z}{d}\right) e^{ \pm \mathrm{i} m \phi-\mathrm{i} \omega t}
$$


where $m$ is an integer ranging from 0 to $\infty, n$ is an integer ranging from 1 to $\infty$, $E_{0}$ is the on-axis electric field, $J_{m}$ is the bessel function of order $m, r$ is the radial coordinate, and $\gamma_{m n}$ is given by

$$
\gamma_{m n}=\sqrt{\mu_{0} \epsilon_{0} \omega_{m n p}^{2}-\left(\frac{p \pi}{d}\right)^{2}}=\frac{x_{m n}}{b},
$$

where $x_{m n}$ is the $n$th root of the equation $J_{m}(x)=0$, and $b$ is the inner radius of the cavity shown in Figure 3.1.

The resonance frequency of the lowest transverse magnetic mode is obtained by substituting 0,1 , and 0 for $m, n$, and $p$ respectively:

$$
\omega_{010}=\frac{2.405}{\sqrt{\mu_{0} \epsilon_{0}} b} .
$$

The inner radius of the cavity, $b$, is chosen to be $142.55 \mathrm{~mm}$, so that that the frequency equals $805 \mathrm{MHz}$. The electric field becomes

$$
E_{z}=E_{0} J_{0}(\gamma r) e^{-\mathrm{i} \omega t}
$$

where $\gamma$ is the first root of the zeroth-order Bessel function divided by the inner radius of the cavity $(\gamma \approx 2.405 / b)$. Solving for the magnetic field in the same manner as the electric field, we get

$$
H_{\phi}=-i \sqrt{\frac{\epsilon_{0}}{\mu_{0}}} E_{0} J_{1}(\gamma r) e^{-\mathrm{i} \omega t},
$$

where $\phi$ represents the azimuthal direction shown in Figure 3.1.

The quantity $E_{z}$ in Equation (3.15) is maximum when $r=0$ (the Bessel function is maximum and equals 1 at $r=0$ ), which means that the maximum electric field is equal to the on-axis electric field. Equation (3.15) also shows that the electric field is independent of the length of the cavity, $d$, so the maximum electric field at the window is equal to the on-axis electric field. In the open $805 \mathrm{MHz}$ cavity, the surface electric field enhancement, $\zeta$, is about 2.6. $\zeta$ is defined as the ratio of the maximum 
surface electric field to the on-axis electric field. Hence, closing the cavity ends by electrically conducting windows decreases the surface electric field enhancement to 1 .

The windows and the cavity are heated from the RF power. The general form of the RF power dissipated in the conductors is given by Equation (3.7). Referring to Figure 3.1, let the subscript $I$ refers to the beryllium window, the subscript $I I$ to the window frame, and the subscript III to the cavity wall. Then the power loss in each part is given by

$$
\begin{gathered}
P_{I}(r)=\frac{\pi F R_{I}}{2} \frac{\epsilon_{0}}{\mu_{0}} E_{0}^{2}\left[r^{2} J_{0}^{2}(\gamma r)+r^{2} J_{1}^{2}(\gamma r)-\frac{2 r J_{0}(\gamma r) J_{1}(\gamma r)}{\gamma}\right] \\
P_{I I}(r)=\frac{\pi F R_{I I}}{2} \frac{\epsilon_{0}}{\mu_{0}} E_{0}^{2}\left[r^{2} J_{0}^{2}(\gamma r)+r^{2} J_{1}^{2}(\gamma r)-\frac{2 r J_{0}(\gamma r) J_{1}(\gamma r)}{\gamma}\right] \\
-\frac{\pi F R_{I I}}{2} \frac{\epsilon_{0}}{\mu_{0}} E_{0}^{2}\left[a^{2} J_{0}^{2}(\gamma a)+a^{2} J_{1}^{2}(\gamma a)-\frac{2 a J_{0}(\gamma a) J_{1}(\gamma a)}{\gamma}\right] . \\
P_{I I I}(r)=\pi F b d R_{I I I} \frac{\epsilon_{0}}{\mu_{0}} E_{0}^{2} J_{1}^{2}(2.405),
\end{gathered}
$$

where $R_{I}, R_{I I}$, and $R_{I I I}$ are the window surface resistance, frame surface resistance, and cavity wall surface resistance respectively.

The total power loss, $P_{t}$, dissipated in the cavity and the windows is given by

$$
P_{t}=2 P_{I}+2\left(P_{I I}\right)+P_{I I I}(b)
$$

The general form of the RF power loss density is given by Equation (3.8). The power loss density function, $q$, for each part is given by

$$
\begin{gathered}
q_{I}(r)=\frac{F R_{I}}{2} \frac{\epsilon_{0}}{\mu_{0}} E_{0}^{2} J_{1}^{2}(\gamma r) \\
q_{I I}(r)=\frac{F R_{I I}}{2} \frac{\epsilon_{0}}{\mu_{0}} E_{0}^{2} J_{1}^{2}(\gamma r) \\
q_{I I I}(r)=\frac{F R_{I I I}}{2} \frac{\epsilon_{0}}{\mu_{0}} E_{0}^{2} J_{1}^{2}(2.405) .
\end{gathered}
$$


The total stored energy in the cavity, $U_{t}$, is given by

$$
U_{t}=\frac{1}{2} \int_{V} \epsilon_{0}\left|E_{z}\right|^{2} \mathrm{~d} V
$$

where $V$ is the volume of the cavity. Substituting Equation (3.15) into Equation (3.24) gives

$$
U_{t}=\frac{\pi d E_{0}^{2} b^{2} \epsilon_{0}}{2}\left[J_{1}^{2}(2.405)\right]
$$

The quality factor is a measure of the sharpness in frequency of the cavity response to the external excitation. It is given by

$$
Q_{f}=\omega_{010} \frac{U_{t}}{P_{t}}
$$

Considering one-dimensional heat conduction in the radial direction in the beryllium foil and the copper frame, the energy balance can be written as

$$
Q_{r}+q \mathrm{~d} A_{s}=Q_{r}+\frac{\mathrm{d} Q_{r}}{\mathrm{~d} r} \mathrm{~d} r
$$

where $Q_{r}$ is the radial heat flow and $A_{s}$ is the surface area subjected to the power loss density $\left(A_{s}=\pi r^{2}\right)$. Fourier's law implies that

$$
Q_{r}=-k A_{c} \frac{\mathrm{d} T}{\mathrm{~d} r}
$$

where $k$ is the thermal conductivity of the material, $T$ is the temperature, and $A_{c}$ is the cross sectional area normal to the heat flow direction, i.e., $A_{c}=2 \pi r t$, where $t$ is the thickness of the material. Substituting Equation (3.28) into Equation (3.27) yields

$$
2 \pi q r \mathrm{~d} r=-2 \pi k \frac{\mathrm{d}}{\mathrm{d} r}\left(r t \frac{\mathrm{d} T}{\mathrm{~d} r}\right) \mathrm{d} r
$$

Integrating Equation (3.29) with respect to $r$ over the area of the beryllium foil yields

$$
\frac{\mathrm{d} T_{I}}{\mathrm{~d} r}=\frac{c_{1}}{2 \pi r t_{I} k_{I}}-\frac{F R_{I} \epsilon_{0} E_{0}^{2}}{4 \mu_{0} t_{I} k_{I}}\left[r J_{0}^{2}(\gamma r)+r J_{1}^{2}(\gamma r)-2 \frac{J_{0}(\gamma r) J_{1}(\gamma r)}{\gamma}\right] .
$$


Integrating one more time with respect to $r$ yields

$$
\begin{aligned}
T_{I}(r)= & -\frac{F R_{I}}{4 t_{I} k} \frac{\epsilon_{0}}{\mu_{0}} E_{0}^{2}\left[r^{2} J_{0}^{2}(\gamma r)+r^{2} J_{1}^{2}(\gamma r)-\frac{r J_{0}(\gamma r) J_{1}(\gamma r)}{\gamma}+\frac{J_{0}^{2}(\gamma r)-1}{\gamma^{2}}\right] \\
& +\frac{c_{1}}{2 \pi t_{I} k_{I}} \ln (r)+c_{2} .
\end{aligned}
$$

Next, integrating Equation (3.29) with respect to $r$ over the area of the cavity frame yields

$$
\frac{\mathrm{d} T_{I I}}{\mathrm{~d} r}=\frac{c_{3}}{2 \pi r t_{I I} k_{I I}}-\frac{F R_{I I} \epsilon_{0} E_{0}^{2}}{4 \mu_{0} t_{I I} k_{I I}}\left[r J_{0}^{2}(\gamma r)+r J_{1}^{2}(\gamma r)-2 \frac{J_{0}(\gamma r) J_{1}(\gamma r)}{\gamma}\right],
$$

and integrating one more time with respect to $r$ gives

$$
\begin{aligned}
T_{I I}(r)= & -\frac{F R_{I I}}{4 t_{I I} k} \frac{\epsilon_{0}}{\mu_{0}} E_{0}^{2}\left[r^{2} J_{0}^{2}(\gamma r)+r^{2} J_{1}^{2}(\gamma r)-r \frac{J_{0}(\gamma r) J_{1}(\gamma r)}{\gamma}+\frac{J_{0}^{2}(\gamma r)}{\gamma^{2}}\right] \\
& +\frac{c_{3}}{2 \pi t_{I I} k_{I I}} \ln (r)+c_{4},
\end{aligned}
$$

where $c_{1}, \ldots, c_{4}$ are constants to be obtained using the following boundary conditions. The temperature at the center of the beryllium foil is finite, which implies that

$$
\frac{\mathrm{d} T_{I}}{\mathrm{~d} r}(r=0)=0
$$

At the contact point between the beryllium foil and the frame, the temperature of the beryllium foil equals the temperature of the copper frame:

$$
T_{I}(r=a)=T_{I I}(r=a) .
$$

At the contact point between the beryllium foil and the cavity frame, the rate of heat transfer in the beryllium foil equals the rate of heat transfer in the the copper frame:

$$
t_{I} k_{I} \frac{\mathrm{d} T_{I}}{\mathrm{~d} r}(r=a)=t_{I I} k_{I I} \frac{\mathrm{d} T_{I I}}{\mathrm{~d} r}(r=a)
$$

The cavity wall is assumed to be cooled to a reference temperature, which is taken to be $0{ }^{\circ} \mathrm{C}$. This implies that

$$
T_{I I}(r=b)=0 .
$$


Using the above boundary conditions, the integration constants are determined to be

$$
\begin{gathered}
c_{1}=0 \\
c_{3}=-P_{I}(a)+\frac{\pi F R_{I I} \epsilon_{0} E_{0}^{2}}{2 \mu_{0}}\left[a J_{0}^{2}(\gamma a)+a J_{1}^{2}(\gamma a)-2 \frac{J_{0}(\gamma a) J_{1}(\gamma a)}{\gamma}\right] \\
c_{4}=-c_{3} \frac{\ln b}{2 \pi t_{I I} k_{I I}}+\frac{F R_{I I} \epsilon_{0} E_{0}^{2}}{4 \mu_{0} t_{I I} k_{I I}}\left[b^{2} J_{1}^{2}(2.405)\right] \\
c_{2}=\frac{F R_{I} \epsilon_{0} E_{0}^{2}}{4 t_{I} k_{I} \mu_{0}}\left[a^{2} J_{0}^{2}(\gamma a)+a^{2} J_{1}^{2}(\gamma a)-a \frac{J_{0}(\gamma a) J_{1}(\gamma a)}{\gamma}+\frac{J_{0}^{2}(\gamma a)-1}{\gamma^{2}}\right] \\
-\frac{F R_{I I} \epsilon_{0} E_{0}^{2}}{4 t_{I I} k_{I I} \mu_{0}}\left[a^{2} J_{0}^{2}(\gamma a)+a^{2} J_{1}^{2}(\gamma a)-a \frac{J_{0}(\gamma a) J_{1}(\gamma a)}{\gamma}+\frac{J_{0}^{2}(\gamma a)}{\gamma^{2}}\right] \\
+\frac{c_{3}}{2 \pi t_{I I} k_{I I}} \ln a+c_{4}+\frac{c_{3}}{2 \pi t_{I I} k_{I I}} \ln a .
\end{gathered}
$$

The exact solutions obtained in this section will be compared to finite element approximations that will be obtained in the next section.

\subsection{Finite Element Solutions}

The volume of the cavity is meshed with electromagnetic elements to perform a modal electromagnetic analysis. The electromagnetic mesh is shown in Figure 3.2, where the elements are chosen to be three-dimensional tetrahedral solid elements. The relative element size with respect to the inner radius of the cavity, $r_{e} / b$, equals about 0.03 , and the total number of elements is about 70,000. The frequency, electromagnetic fields, quality factor, and stored energy were obtained. The windows and the copper frame were then meshed with thermal elements to obtain the power loss density and the temperature profile. The thermal surface mesh is compatible with the electromagnetic mesh to allow for the transfer of power loss density results from the electromagnetic elements to the thermal elements. The thermal surface mesh shown in Figure 3.2 is composed of about 2000 3-node shell elements.

The finite element procedure for getting the temperature field in the windows and the frames is a two-step process. The first step is to solve for the electric and 
magnetic fields, and to determine the surface flux distribution on the relevant surfaces of the cavity. The next step is to perform a thermal analysis on the cavity using 3node shell elements that are compatible with the tetrahedral elements used in the electromagnetic analysis.

The cylindrical pillbox cavity considered in this chapter responds to RF power by resonating in a particular mode of oscillation that has an electric field component along the axial direction of the cavity and a cylindrical magnetic field around the inside of the cavity. The particles will gain energy from the electric field. The magnetic field provides an inward force towards the center of the cavity that compensates for the electrical repulsion among the particles in a bunch. The beam is timed to enter the cavity just when the electric field force is close to its maximum and pointing in the axial direction.

Figure 3.3 shows that the finite element electric field distribution is smooth. It is maximum at the center of the cavity, and decreases gradually along the radius toward the cylindrical wall of the cavity. Figure 3.4 shows the finite element magnetic field distribution, which is also smooth. It is negligible at the center of the cavity and increases gradually along the radius toward the cylindrical wall of the cavity.

The power loss density obtained from the finite element results is shown in Figure 3.5. It is observed from the figure that the power loss density is negligible at the center of the cavity and increases gradually along the radius toward the cylindrical wall of the cavity. There is a discontinuity in the power loss density at the contact point between the beryllium foil and the copper frame because the electrical conductivity of the copper is higher than that of the beryllium. The power loss density is modeled as a heat flux distribution that acts on the conductor surfaces. This procedure is valid only if the conductor thickness is much greater than the skin depth, as in the present case. Figure 3.6 shows the temperature rise, which is maximum at 
the center of the window and decreases along the radius toward the wall of the cavity.

Comparison between the analytical and finite element solutions of the electric field, magnetic field, power loss density, and temperature are presented in Figures $(3.7-3.10)$. Excellent agreement is obtained between the analytical and the finite element solutions for all the quantities. The electromagnetic and thermal results are summarized in Table 3.2, where both analytical and finite element results are presented. The table shows that the finite element procedure performed in this section is an accurate and effective tool for the electromagnetic and thermal design of RF windows.
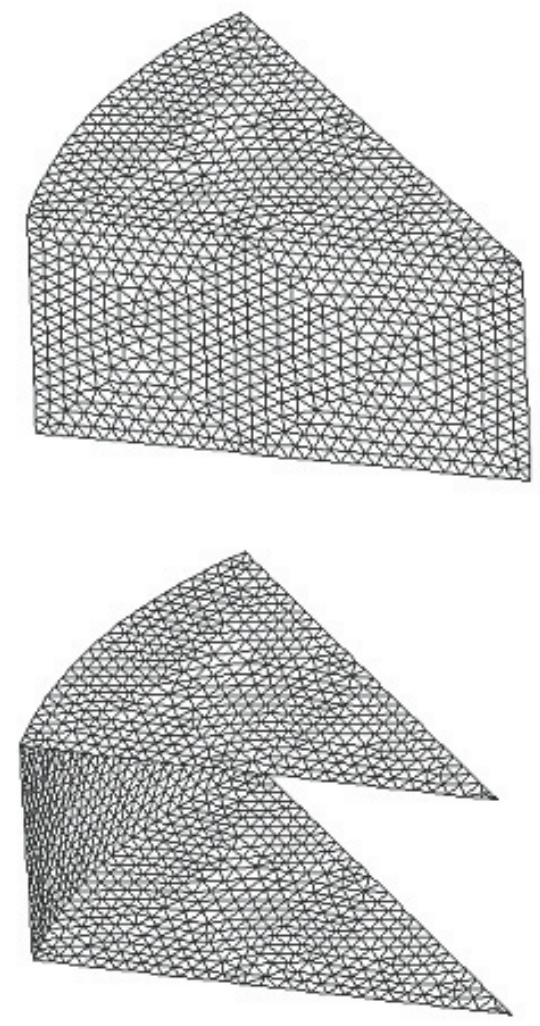

Figure 3.2. Electromagnetic Volume Mesh of the Cylindrical Pillbox Cavity and Thermal Surface Mesh of the Cavity and the Windows. (Relative Element Size with Respect to the Inner Radius of the Cavity, $r_{e} / b$, Equals About 0.03). 


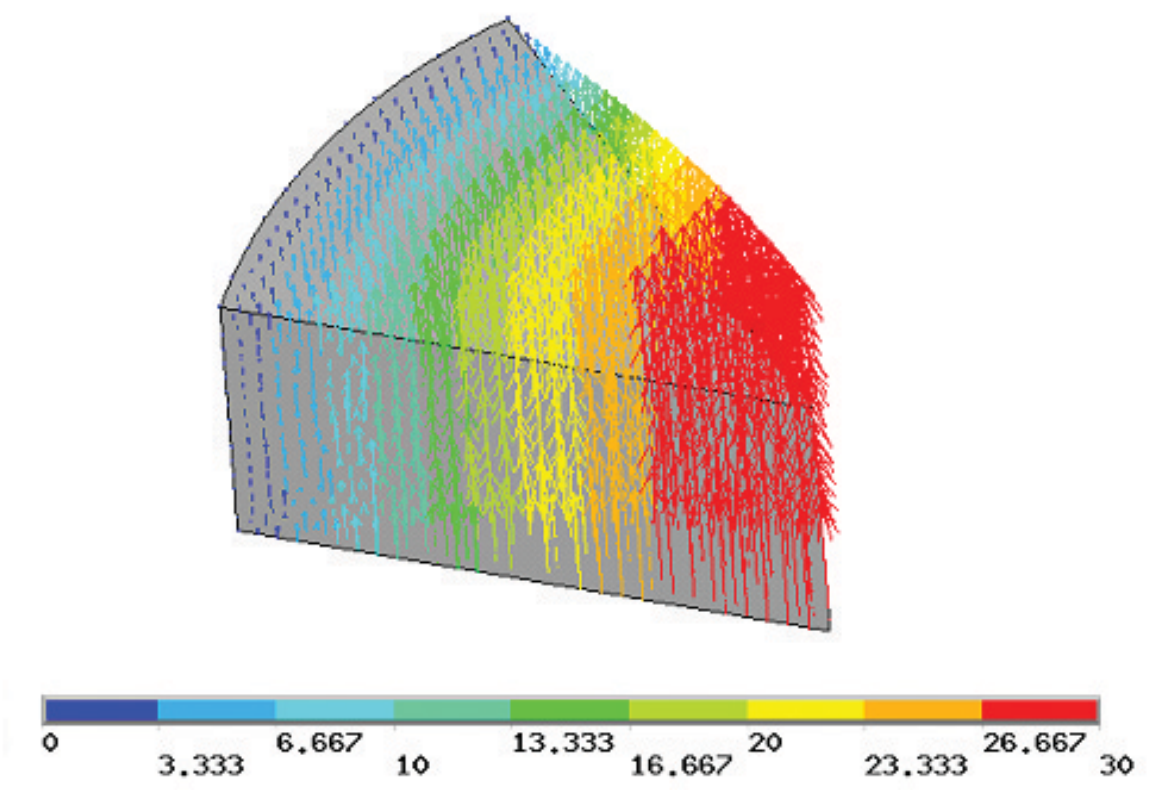

Figure 3.3. Vector Plot of the Electric Field in the Cylindrical Pillbox Cavity $(\mathrm{MV} / \mathrm{m})$. On-Axis Electric Field $=30 \mathrm{MV} / \mathrm{m}$.
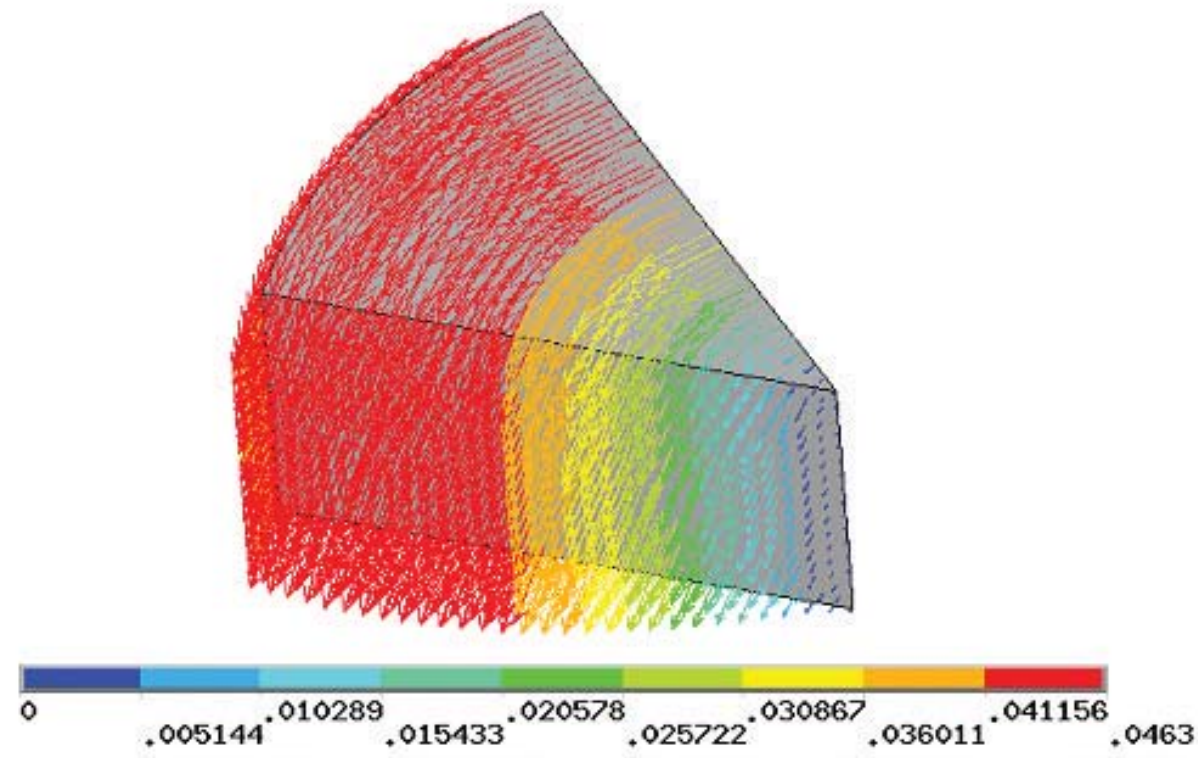

Figure 3.4. Vector Plot of the Magnetic Field in the Cylindrical Pillbox Cavity $(\mathrm{MA} / \mathrm{m})$. On-Axis Electric Field $=30 \mathrm{MV} / \mathrm{m}$. 


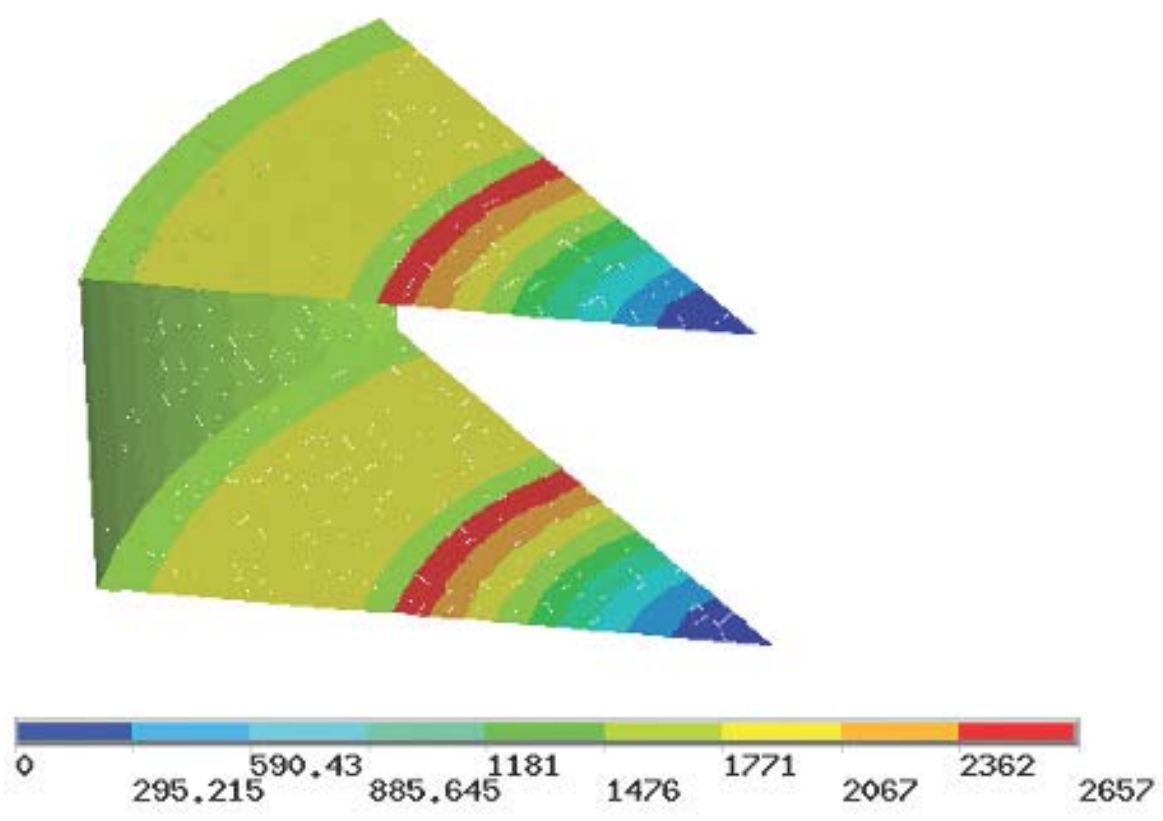

Figure 3.5. Contour Plot of the Power Loss Density in the Cylindrical Pillbox Cavity $\left(\mathrm{W} / \mathrm{m}^{2}\right)$.

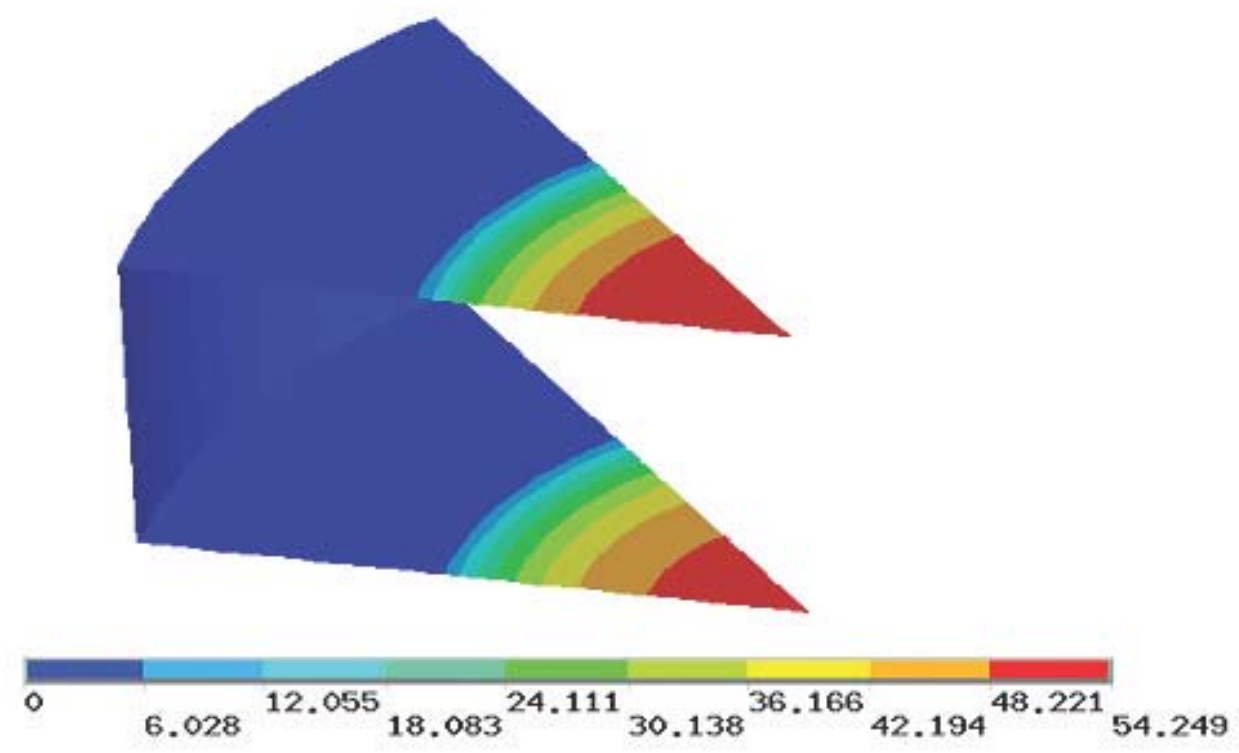

Figure 3.6. Contour plot of the Temperature Distribution in the Cylindrical Pillbox Cavity $\left({ }^{\circ} \mathrm{C}\right)$. 


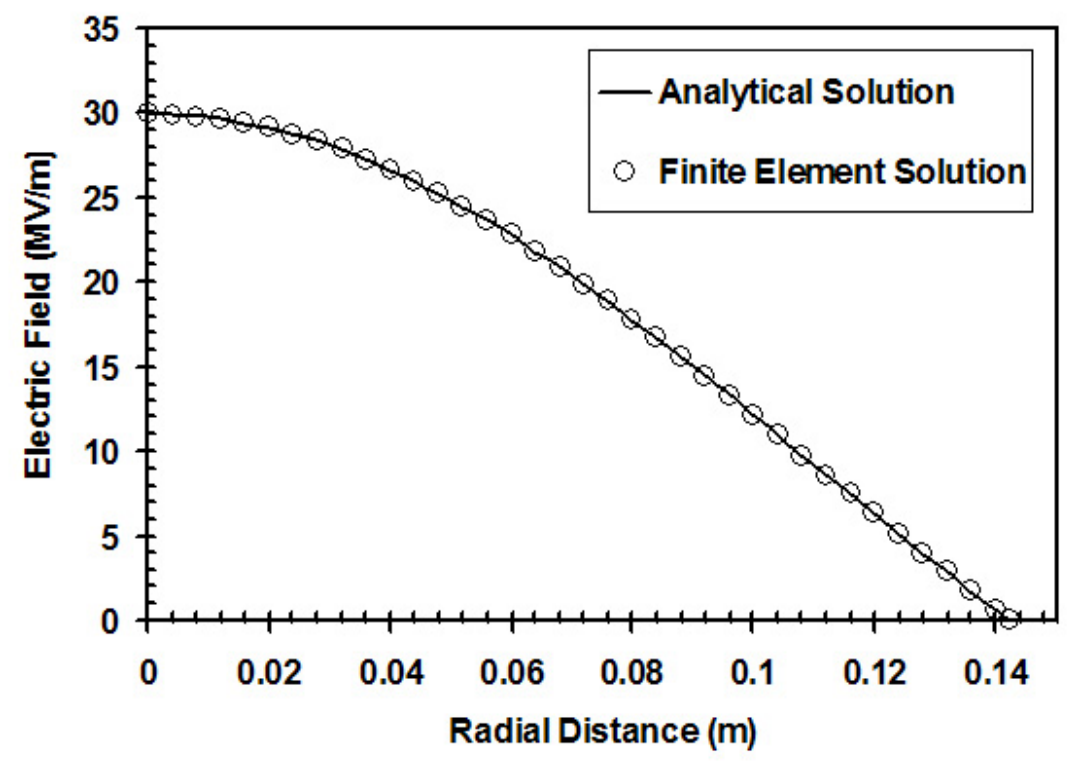

Figure 3.7. Analytical and Finite Element Electric Field Profiles in the Cylindrical Pillbox Cavity.

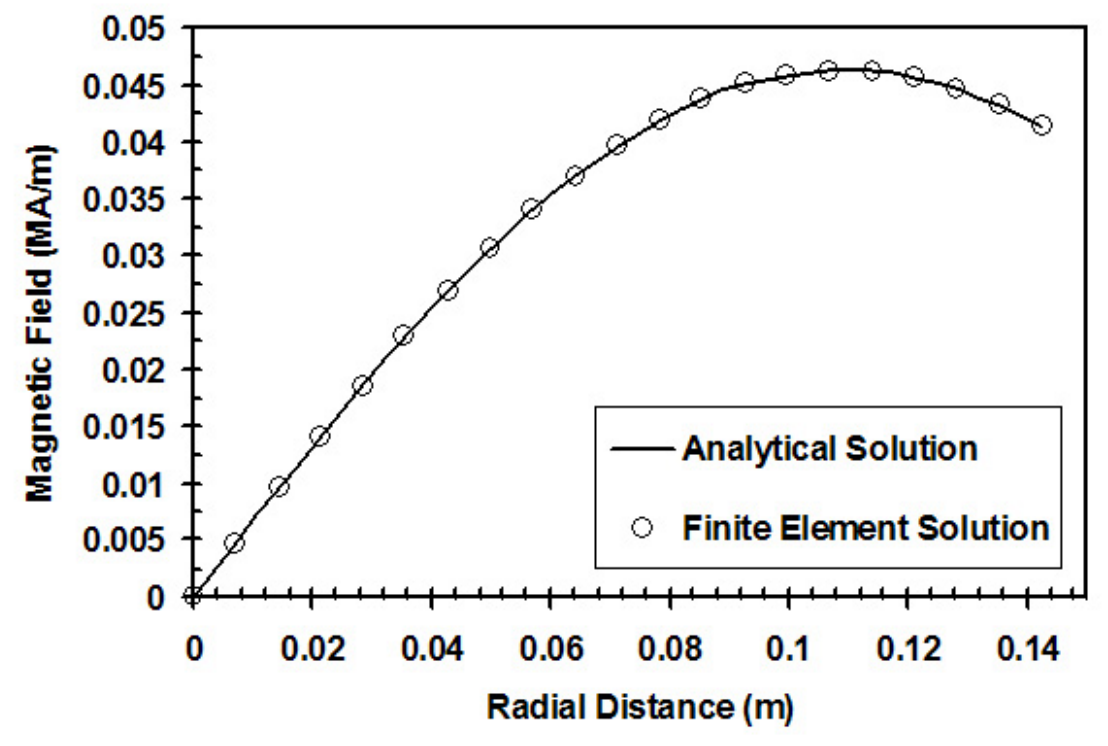

Figure 3.8. Analytical and Finite Element Magnetic Field Profiles in the Cylindrical Pillbox Cavity. 


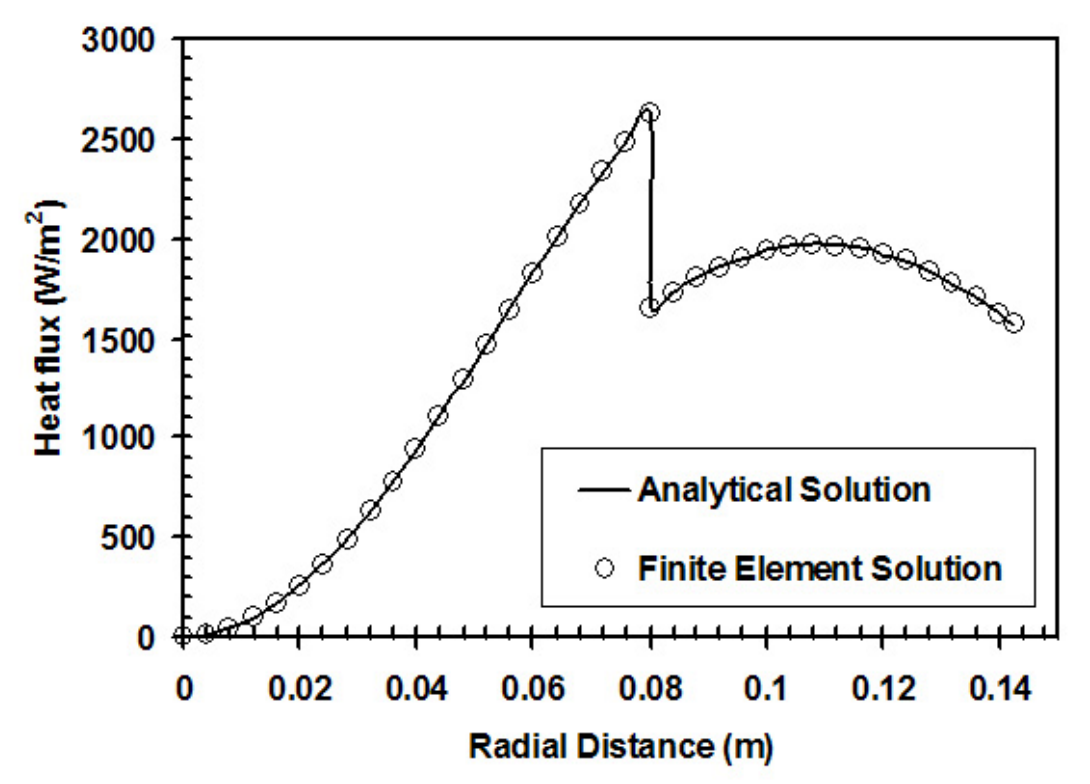

Figure 3.9. Analytical and Finite Element Power Loss Density Profiles in the Cylindrical Pillbox Cavity.

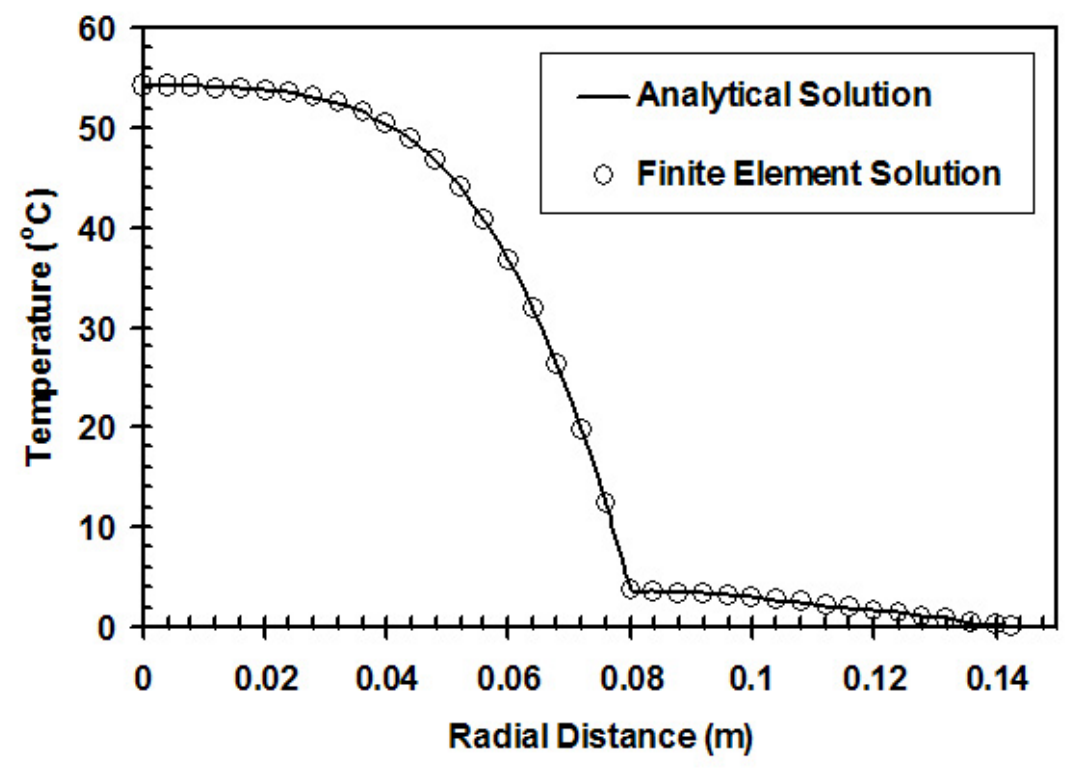

Figure 3.10. Analytical and Finite Element Temperature Profiles in the Cylindrical Pillbox Cavity. 
Table 3.2. Comparison Between the Finite Element and the Analytical Results for the Cylindrical Pillbox Cavity.

\begin{tabular}{ccc}
\hline \hline Parameter & Analytical Value & FEA Value \\
\hline Frequency $(\mathrm{MHz})$ & 805.0 & 805.0 \\
Maximum field enhancement $(\mathrm{MV} / \mathrm{m})$ & 1.0 & 1.0 \\
Quality factor & 20260.9 & 20271.3 \\
Stored energy $(\mathrm{J})$ & 0.0013 & 0.0013 \\
Maximum heat flux-window $\left(\mathrm{W} / \mathrm{m}^{2}\right)$ & 2626.5 & 2634.7 \\
Maximum heat flux-frame $\left(\mathrm{W} / \mathrm{m}^{2}\right)$ & 1651.5 & 1659.2 \\
Maximum temperature-window $\left({ }^{\circ} \mathrm{C}\right)$ & 54.16 & 54.17 \\
Maximum temperature-frame $\left({ }^{\circ} \mathrm{C}\right)$ & 3.84 & 3.84 \\
\hline
\end{tabular}




\section{CHAPTER 4 \\ FINITE ELEMENT ANALYSIS OF THE TEST CAVITY WITH BERYLLIUM WINDOWS}

In this chapter, the test cavity closed by the beryllium windows is studied. In Section 4.1, electromagnetic finite element analysis is performed, where frequency, electromagnetic fields, ratio of the peak surface electric field to the on-axis electric field, and quality factor are calculated. In Section 4.2, thermal finite element analysis is performed based on the electromagnetic results, where power loss density and temperature profile are calculated.

\subsection{Electromagnetic Analysis}

The $805 \mathrm{MHz}$ test cavity described in Section 2.1 was considered. The cavity ends were closed by the beryllium windows as shown in Figure 2.1. The electromagnetic finite element mesh of the vacuum inside the cavity is shown in Figure 4.1. Three-dimensional tetrahedral solid elements were employed. A fine mesh near the vacuum boundaries was used to accurately capture both the electromagnetic fields and the thermal results in these regions. The relative minimum electromagnetic element size with respect to the inner radius of the cavity, $r_{e} / b$, equals about 0.003 , and the total number of elements is about 70,000 .

The finite element solution of the electric field is shown in Figure 4.2. The overall general electric field trend is similar to that in the cylindrical pillbox cavity. The electric field is high on the axis of the cavity and decreases gradually along the radius toward the cylindrical walls of the cavity. The electric field takes curved contours in the vicinity of the curved surfaces. It is obvious that the maximum electric field at the window is approximately equal to the on-axis electric field. The surface electric field enhancement at the window, defined as the ratio of the maximum electric field at the window surface to the on-axis electric field, was about 1.006. This has 
been considered as one of the advantages of closing the cavity ends with RF windows. The maximum electric field in the cavity occurs at the curved walls of the cavity as shown in Figure 4.2. The surface electric field enhancement for the cavity, defined as the ratio of the maximum electric field at the cavity to the on-axis electric field, was about 1.107 .

The finite element solution of the magnetic field is shown in Figure 4.3. The overall general magnetic field trend is similar to that in the cylindrical pillbox cavity. It is negligible on the axis of the cavity and increases gradually along the radius of the cavity toward the cylindrical walls of the cavity. The magnetic field takes curved contours in the vicinity of the curved surfaces. The finite element results for the frequency, quality factor, and surface electric field enhancements are shown in Table 4.1 .

Table 4.1. Finite Element Results for the $805 \mathrm{MHz}$ Test Cavity with Beryllium Windows.

\begin{tabular}{ccc}
\hline \hline Parameter & Design Value & FEA Value \\
\hline Frequency $(\mathrm{MHz})$ & 805.0 & 808.3 \\
Quality factor & 18,800 & 18790.844 \\
Maximum field enhancement-window & - & 1.006 \\
Maximum field enhancement-cavity & - & 1.107 \\
Maximum power loss density-window $\left(\mathrm{W} / \mathrm{m}^{2}\right)$ & - & 1918.039 \\
Maximum power loss density-cavity $\left(\mathrm{W} / \mathrm{m}^{2}\right)$ & - & 3187.152 \\
\hline
\end{tabular}



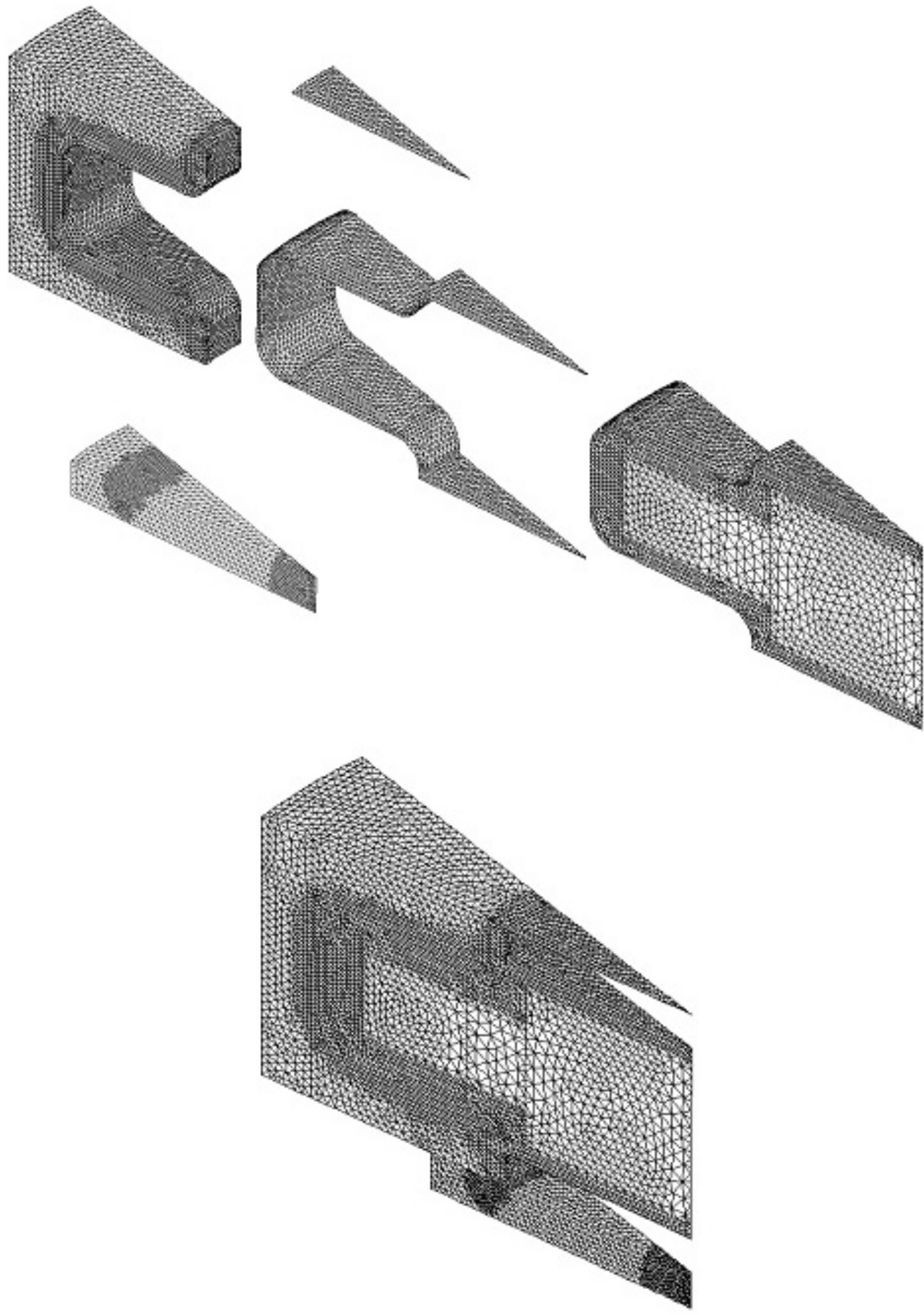

Figure 4.1. Electromagnetic Volume Mesh of the Test Cavity Closed by Beryllium Windows and Thermal Meshes of the Cavity and the Windows (Relative Minimum Element Size with Respect to the Inner Radius of the Cavity, $r_{e} / b$, Equals About $0.003)$. 


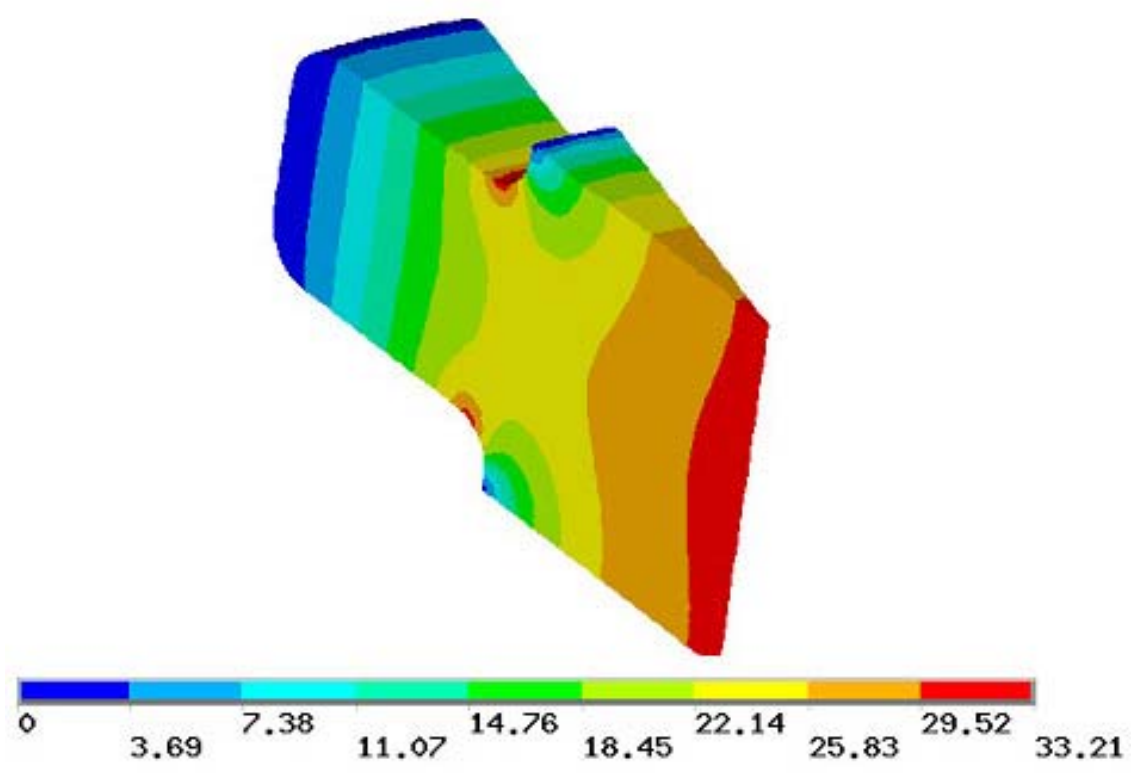

Figure 4.2. Contour Plot of the Electric Field Distribution in the Test Cavity Closed by Beryllium Windows $(\mathrm{MV} / \mathrm{m})$. On-Axis Electric Field $=30 \mathrm{MV} / \mathrm{m}$.

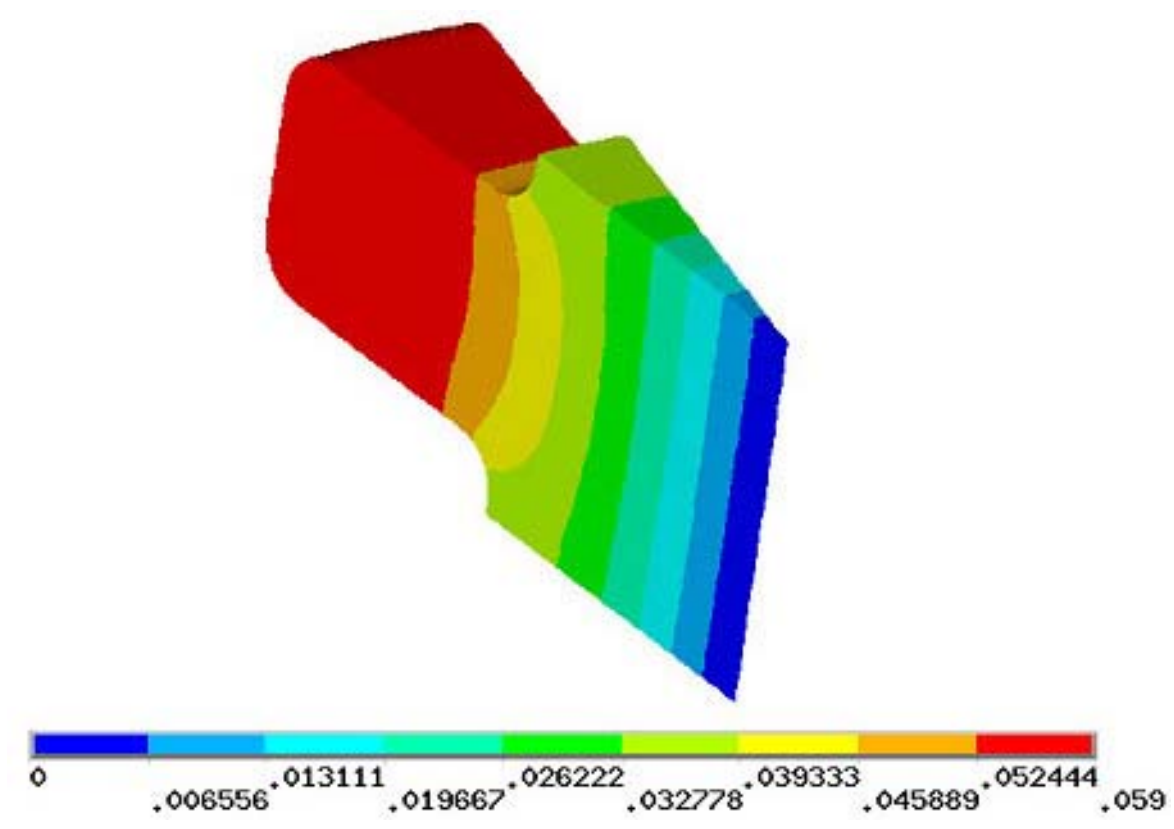

Figure 4.3. Contour Plot of the Magnetic Field Distribution in the Test Cavity Closed by Beryllium Windows (MA/m). On-Axis Electric Field $=30 \mathrm{MV} / \mathrm{m}$. 


\subsection{Thermal Analysis}

The interfacial surfaces between the vacuum and the cavity structures were meshed with 3-node thermal shell elements. The power loss density was transferred from the electromagnetic mesh to the thermal mesh to perform thermal analysis. The electromagnetic mesh was sufficiently refined so as to ensure accurate thermal solution. The thermal mesh is shown in Figure 5.1 where 3-node shell elements were employed to model the beryllium and titanium windows and solid tetrahedral thermal elements were employed to model the other components. The relative minimum thermal element size with respect to the inner radius of the cavity, $r_{e} / b$, equals about 0.003, and the total number of thermal elements is about 145,000 elements. The maximum power loss density in the window and the cavity are presented in Table 4.1

Steady-state heat conduction in the beryllium window was assumed to obtain the temperature distribution. The boundary conditions included the power loss density obtained from the electromagnetic analysis, and a reference temperature $\left(0^{\circ} \mathrm{C}\right)$ specified at the edge of the window. The temperature profile in the window is shown in Figure 4.5. To investigate the temperature distribution at all the components, the reference temperature was assumed to be at the outer boundary of the cavity instead of the edge of the window. The temperature profile is shown in Figures 4.6. It is obvious that the temperature in all of the components except the windows is close to the reference temperature. The temperature profile in the window is quantitatively and qualitatively similar to that obtained in the window of the cylindrical pillbox cavity

considered in Chapter 3. Comparison of some finite element results with experimental results will be discussed later in Chapter 6 . 


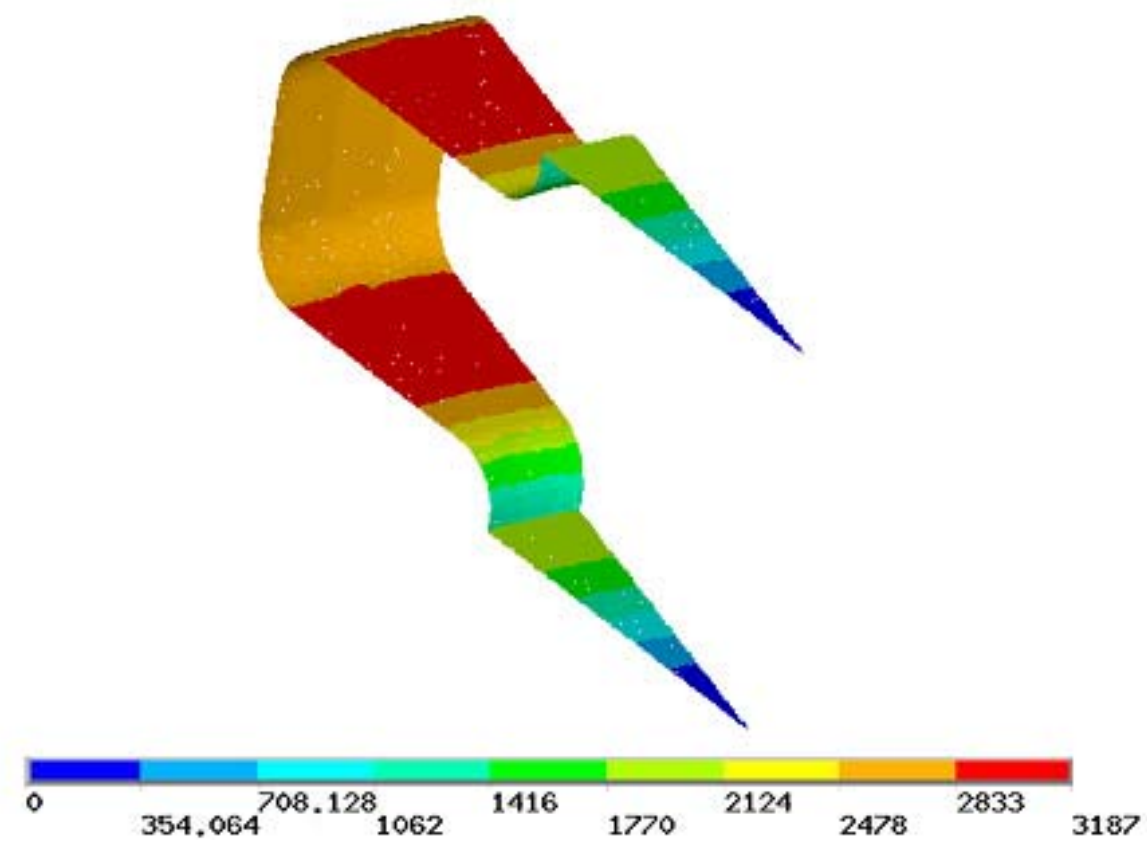

Figure 4.4. Contour Plot of the Power Loss Density in the Test Cavity Closed By Beryllium Windows $\left(\mathrm{W} / \mathrm{m}^{2}\right)$.

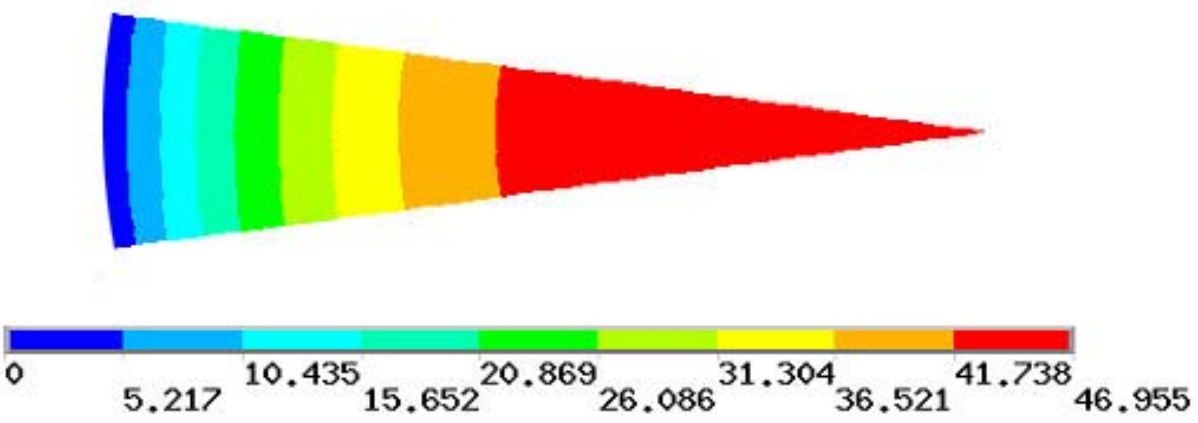

Figure 4.5. Contour Plot of the Temperature Distribution in the Beryllium Window $\left({ }^{\circ} \mathrm{C}\right)$. Edge of the Window is Cooled to a Reference Temperature $\left(0^{\circ} \mathrm{C}\right)$. 


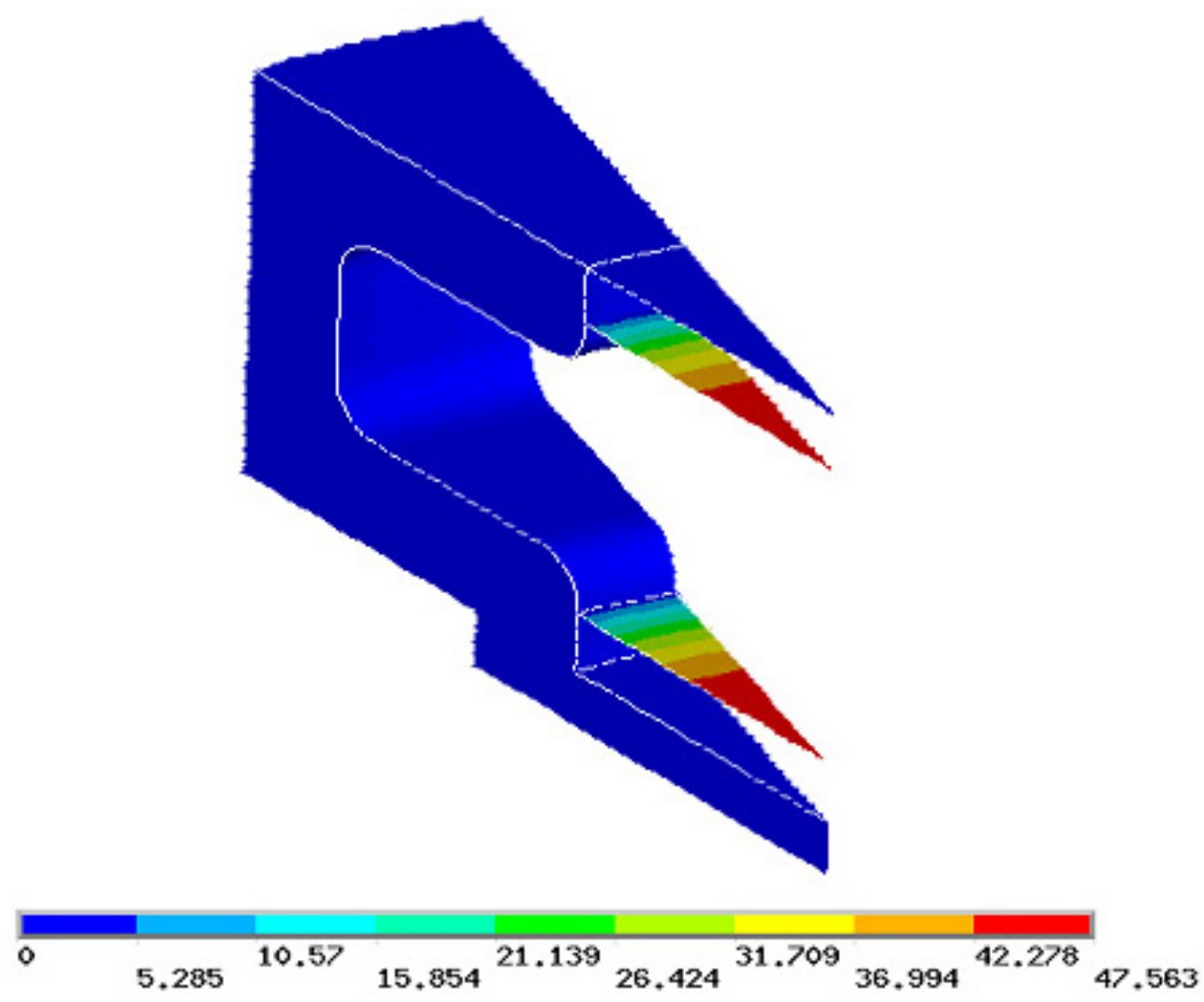

Figure 4.6. Contour Plot of the Temperature Distribution in the Test Cavity Closed by Beryllium Windows $\left({ }^{\circ} \mathrm{C}\right)$. Outer Boundary of the cavity is Cooled to a Reference Temperature $\left(0^{\circ} \mathrm{C}\right)$. 


\section{CHAPTER 5 \\ FINITE ELEMENT ANALYSIS OF THE TEST CAVITY WITH GRIDDED-TUBE WINDOWS}

In this chapter, the test cavity closed by a gridded-tube window from one side, and a beryllium window from the other side is considered. The gridded-tube window is a structure composed of thin-walled aluminum tubes arranged in a particular pattern. In Section 5.1, electromagnetic finite element analysis is performed. Iterative refinement of the mesh is performed in response to the intersectional or contact geometry of the thin-walled tubes. The electric field map, frequency, quality factor, and ratio of the peak surface field to the on-axis field are calculated. In Section 5.2, thermal finite element analysis is performed based on the electromagnetic results, where power loss density and temperature profile are calculated. Forced-convection cooling of the grid via a flow of helium gas inside the tubes is considered in order to reduce the temperature of the tubes. Finally, in Section 5.3, structural analysis is performed to obtain the displacement of the grid, and to perform the corresponding thermal stress analysis.

\subsection{Electromagnetic Analysis}

Figure 5.1 shows the test cavity closed by a gridded-tube window from the upper side, and a beryllium window from the lower side. The titanium window is shown above the gridded-tube window, and the stainless steel plate is shown below the beryllium window. Assuming the axial direction of the cavity is the z-direction, the tubes are then located in the $\mathrm{x}-\mathrm{y}$ plane. The grid configuration shown in Figure 5.1 is a grid of non-touching tubes, composed of an array of tubes parallel to the $\mathrm{x}$ axis and another array of tubes parallel to the y-axis, with a small gap between both arrays. The electric field map for this model is shown in Figure 5.2. One objective of the RF cavity window is to terminate the field. It is obvious that for the present 
configuration, there is a significant field leakage to the region between the grid and the titanium window.

The grid configuration shown in Figure 5.3 is a grid of touching tubes, composed of an array of tubes parallel to the x-axis and another array of tubes parallel to the y-axis, where both arrays are in contact with each other. The electric field map for this model is shown in Figure 5.4. The grid configuration shown in Figure 5.5 is a "waffle" grid, composed of an array of tubes parallel to the x-axis that is intersecting with another array of tubes parallel to the y-axis (both arrays are in the same plane). The electric field map for this model is shown in Figure 5.6. Both the grid of touching tubes and the "waffle" grid show excellent field termination. The finite element results of the electric fields shown in Figures 5.2, 5.4, and 5.6 for the various grid configurations show that the electric field is approximately smooth except in the vicinity of the grid.

The complexity in modeling the grids of thin-walled tubes was due to the intersectional or contact geometry of the tubes, and the wide variation of length scale between the grid wall thickness and the cavity dimensions. One eighth of the cavity was modeled in the case of the "waffle" grid because of the eight-fold symmetry of the "waffle" grid. For the grid of touching tubes, and the grid of non-touching tubes, one quarter of the cavity was modeled because of the four-fold symmetry of both grids. The volume of the cavity was meshed to perform the electromagnetic analysis. The mesh was refined in the vicinity of the tubes to accurately capture the fields in these regions. The electromagnetic results were found to be highly dependent on the mesh density near the curved surfaces and especially near the grid intersectional or contact regions. The electromagnetic volume mesh is shown in Figure 5.5 where three-dimensional tetrahedral solid elements were employed. The relative minimum electromagnetic element size with respect to the inner radius of the cavity, $r_{e} / b$, equals 
about 0.002 , and the total number of electromagnetic elements is about 405,000.

Some elements near abrupt geometrical changes were investigated separately. The fields and power density results were obtained at these regions. The mesh at these regions was then refined. The results were obtained again and compared with the previous results. Based on the variation in the results, some elements were refined again. This iterative meshing process was continued until mesh-independent results were obtained in all the regions in the cavity.

As discussed in Chapter 2, the surface electric field enhancement, defined as the ratio of the maximum surface electric field to the on-axis electric field, should be as low as possible to avoid field emission effects. To investigate this ratio, the electric field distribution was obtained inside the cavity for many grid configurations with different geometrical variables. The results are presented in Table 5.1. It is concluded that increasing the number of tubes, increasing the tube outer diameter, and/or decreasing the spacing of the tubes in the center of the grid reduces this ratio. It is found that the surface electric field enhancement in the grid of non-touching tubes, and the grid of touching tubes are higher than that in the "waffle" grid. Hence, the "waffle" grid was considered as the feasible design candidate from an electromagnetic point of view. The $4 \times 4$ "waffle" grid with tube outer diameter of $9.5 \mathrm{~mm}$ was considered as the baseline gridded-tube window. The detailed geometrical parameters of the baseline gridded-tube window are shown in Table 5.2. The electromagnetic finite element results of the cavity with the baseline gridded-tube window are summarized in Table 5.3 . 


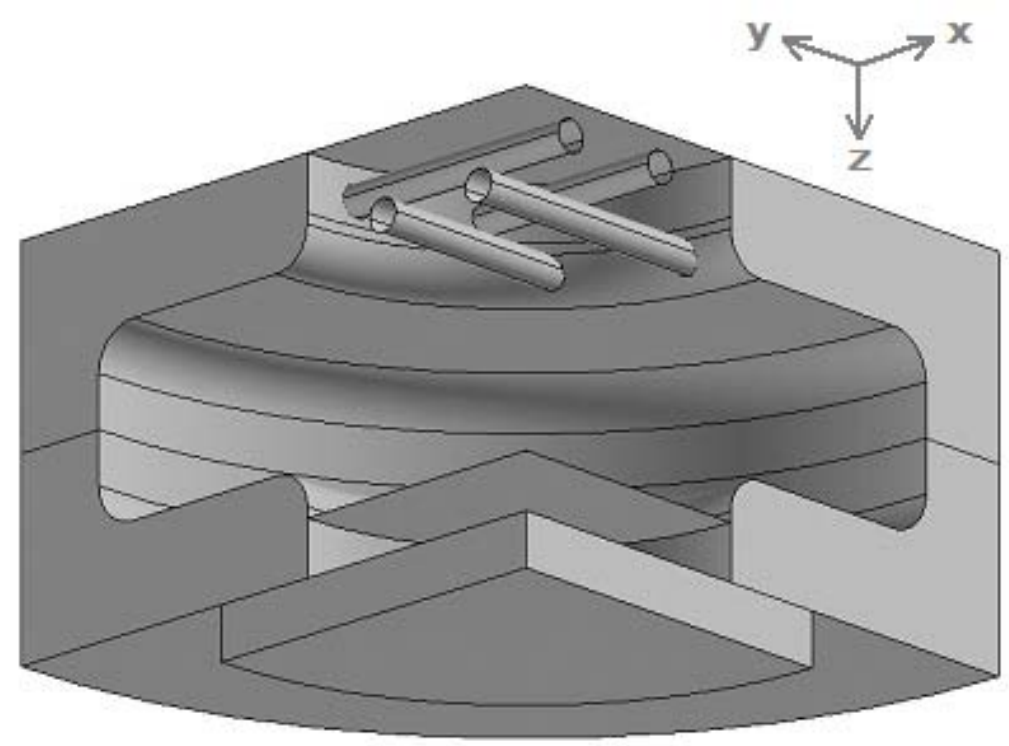

Figure 5.1. The Test Cavity Closed by a Grid of Non-Touching Tubes. Tube Outer Diameter $=9.5 \mathrm{~mm}$.
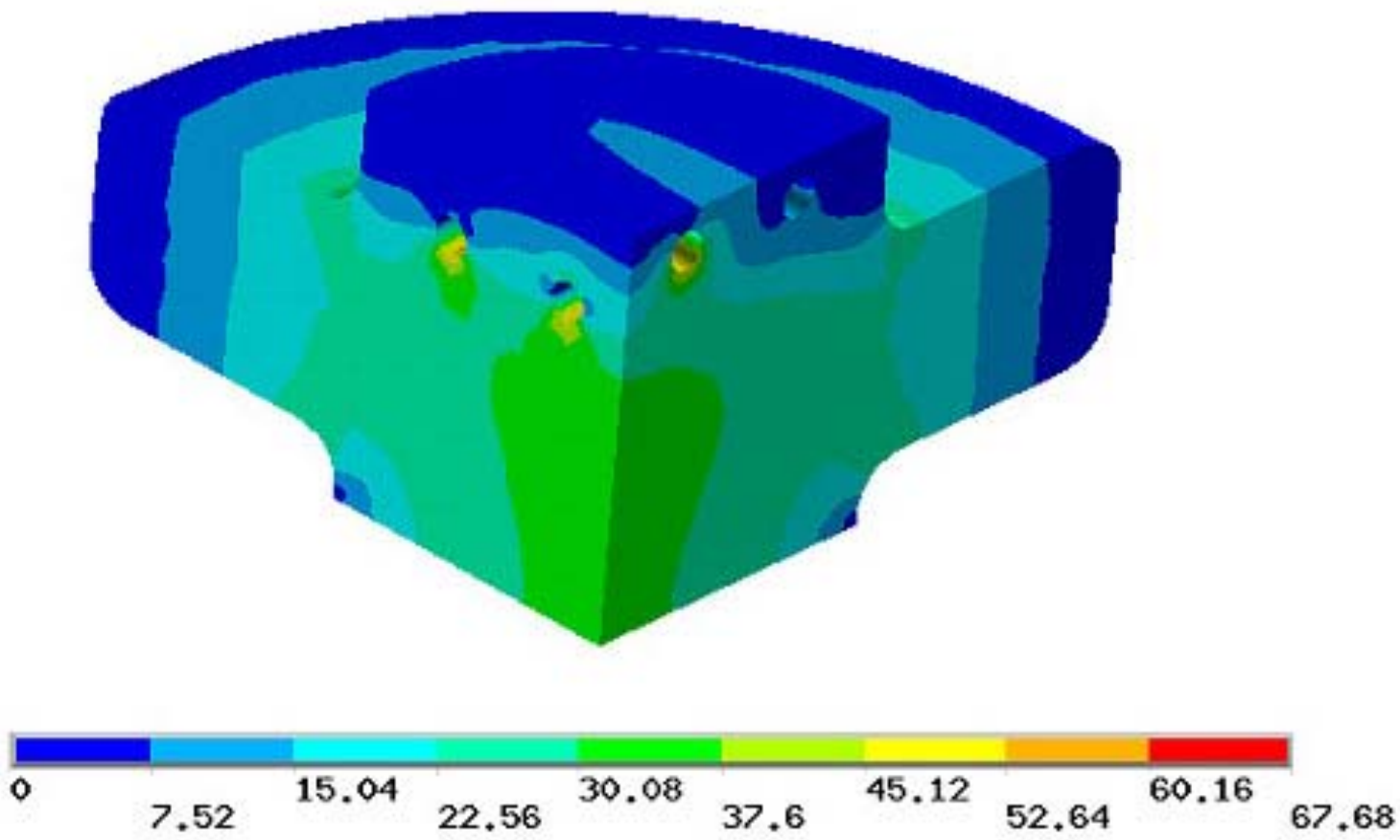

Figure 5.2. Contour Plot of the Electric Field Distribution in the Test Cavity Closed by a Grid of Non-Touching Tubes. Tube Outer Diameter $=9.5 \mathrm{~mm}(\mathrm{MV} / \mathrm{m})$. On-Axis Electric Field $=30 \mathrm{MV} / \mathrm{m}$. 


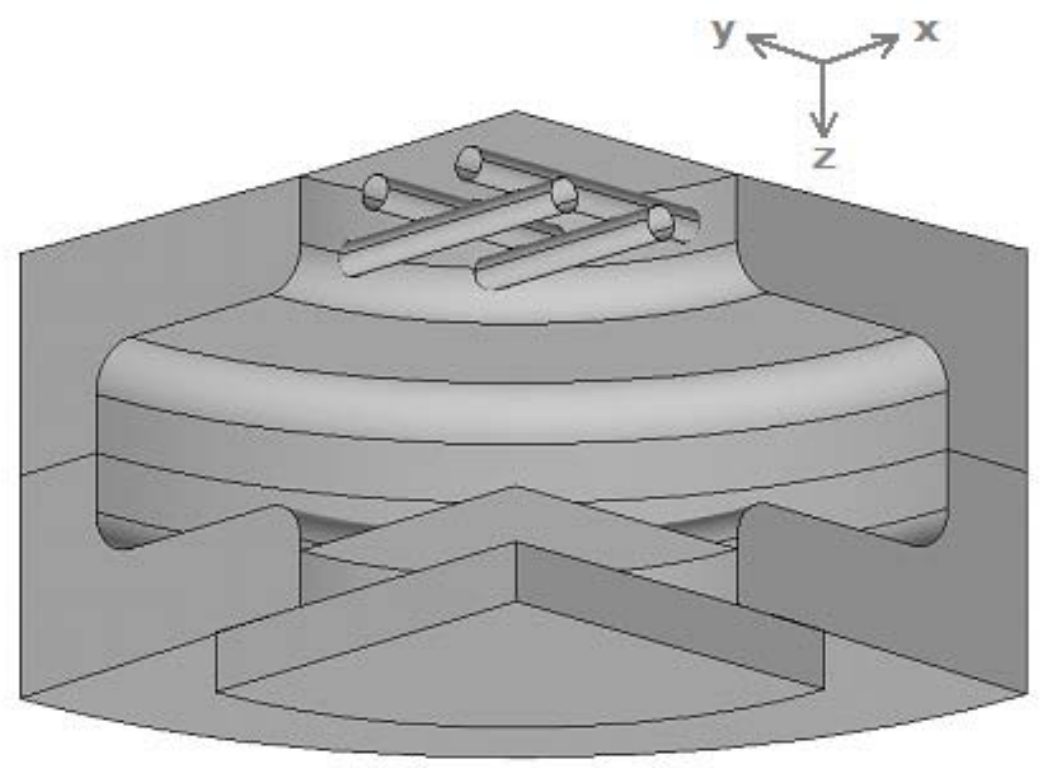

Figure 5.3. The Test Cavity Closed by a Grid of Touching Tubes. Tube Outer Diameter $=9.5 \mathrm{~mm}$.

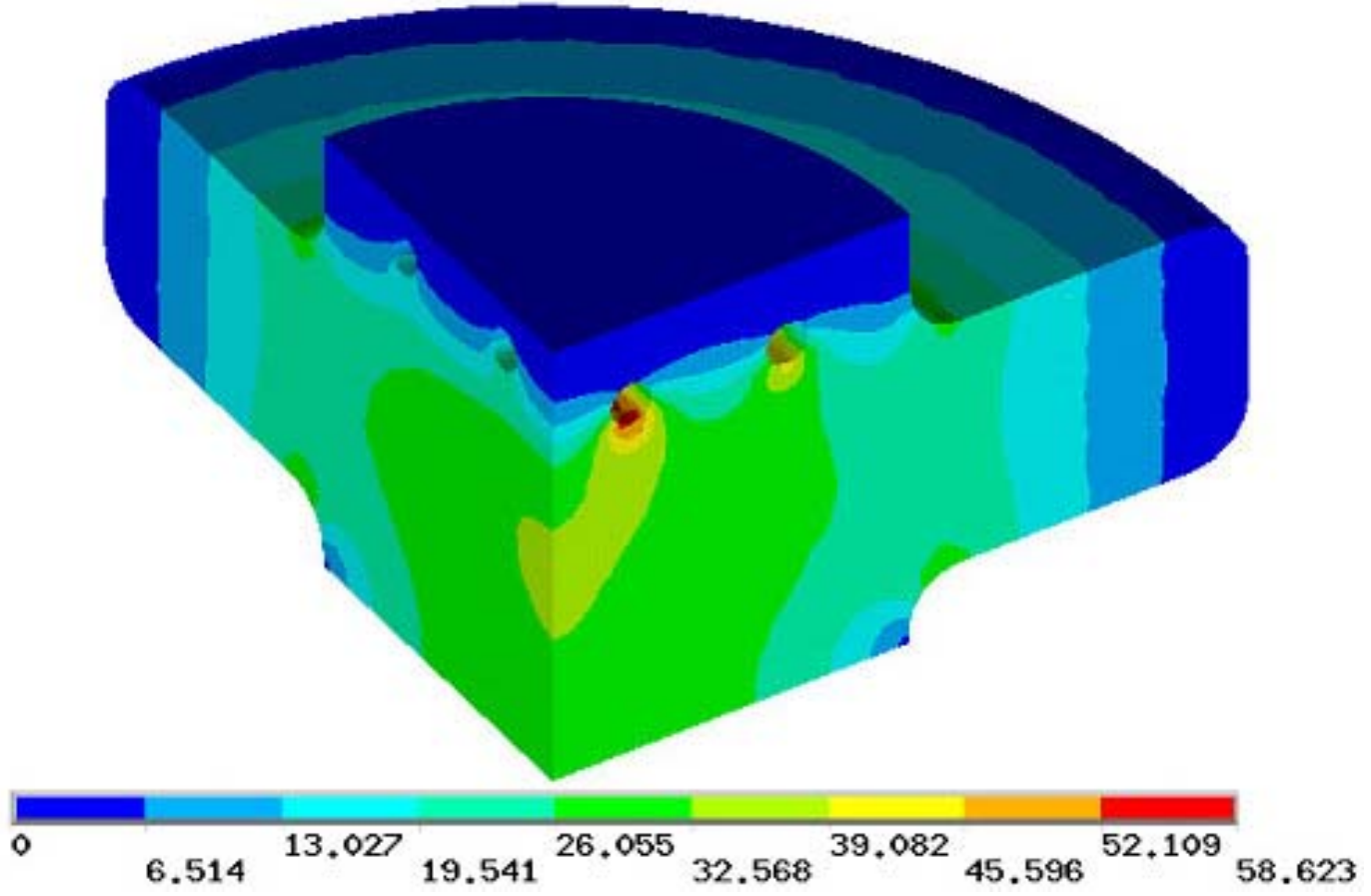

Figure 5.4. Contour Plot of the Electric Field Distribution in the Test Cavity Closed by a Grid of Touching Tubes. Tube Outer Diameter $=9.5 \mathrm{~mm}(\mathrm{MV} / \mathrm{m})$. On-Axis Electric Field $=30 \mathrm{MV} / \mathrm{m}$. 

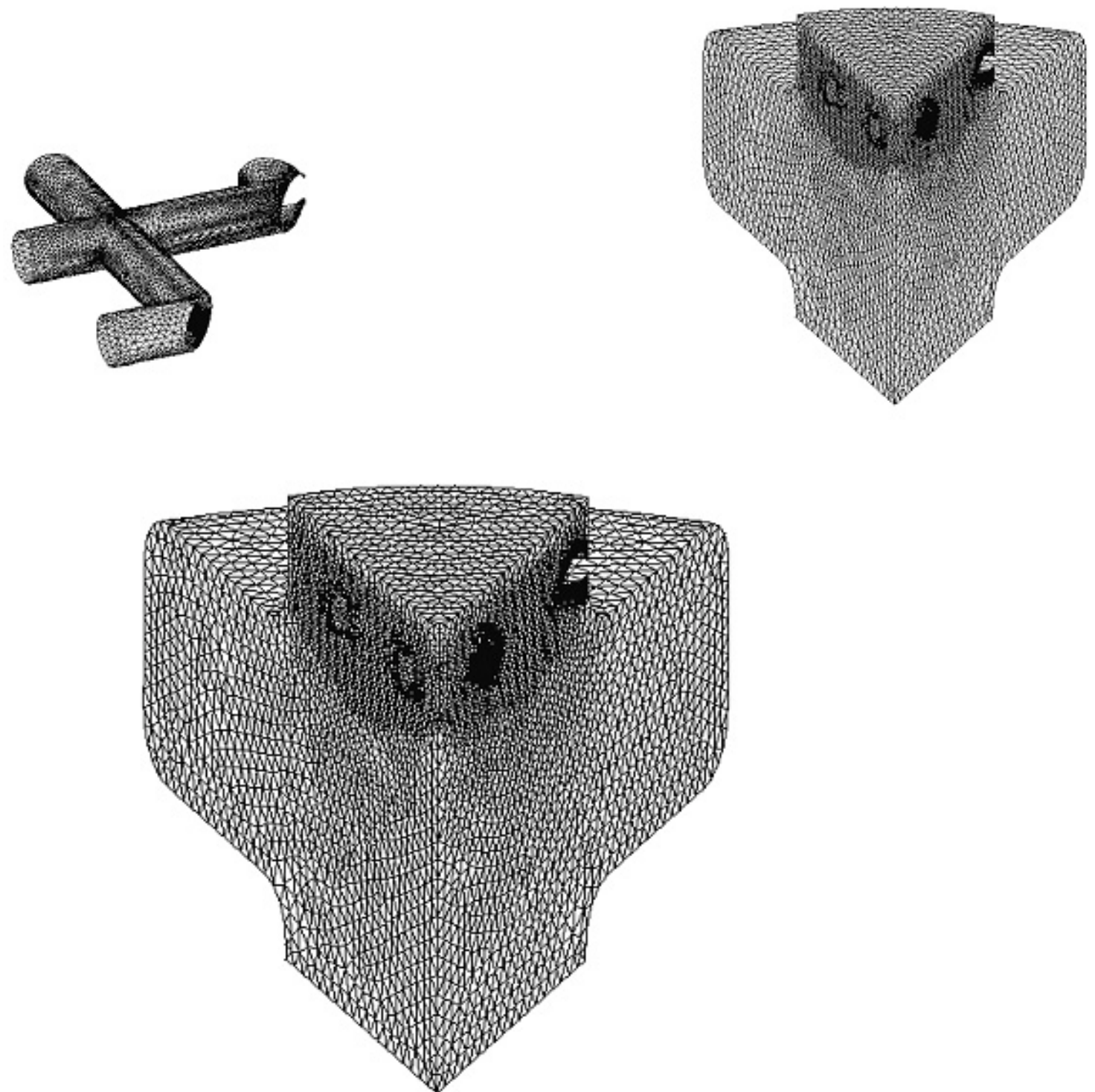

Figure 5.5. Electromagnetic Volume Mesh of Test Cavity Closed by the Baseline Gridded-Tube Window and Thermal Surface Mesh of the Gridded-Tube Window (Relative Minimum Element Size with Respect to the Inner Radius of the Cavity, $r_{e} / b$, Equals About 0.002). 


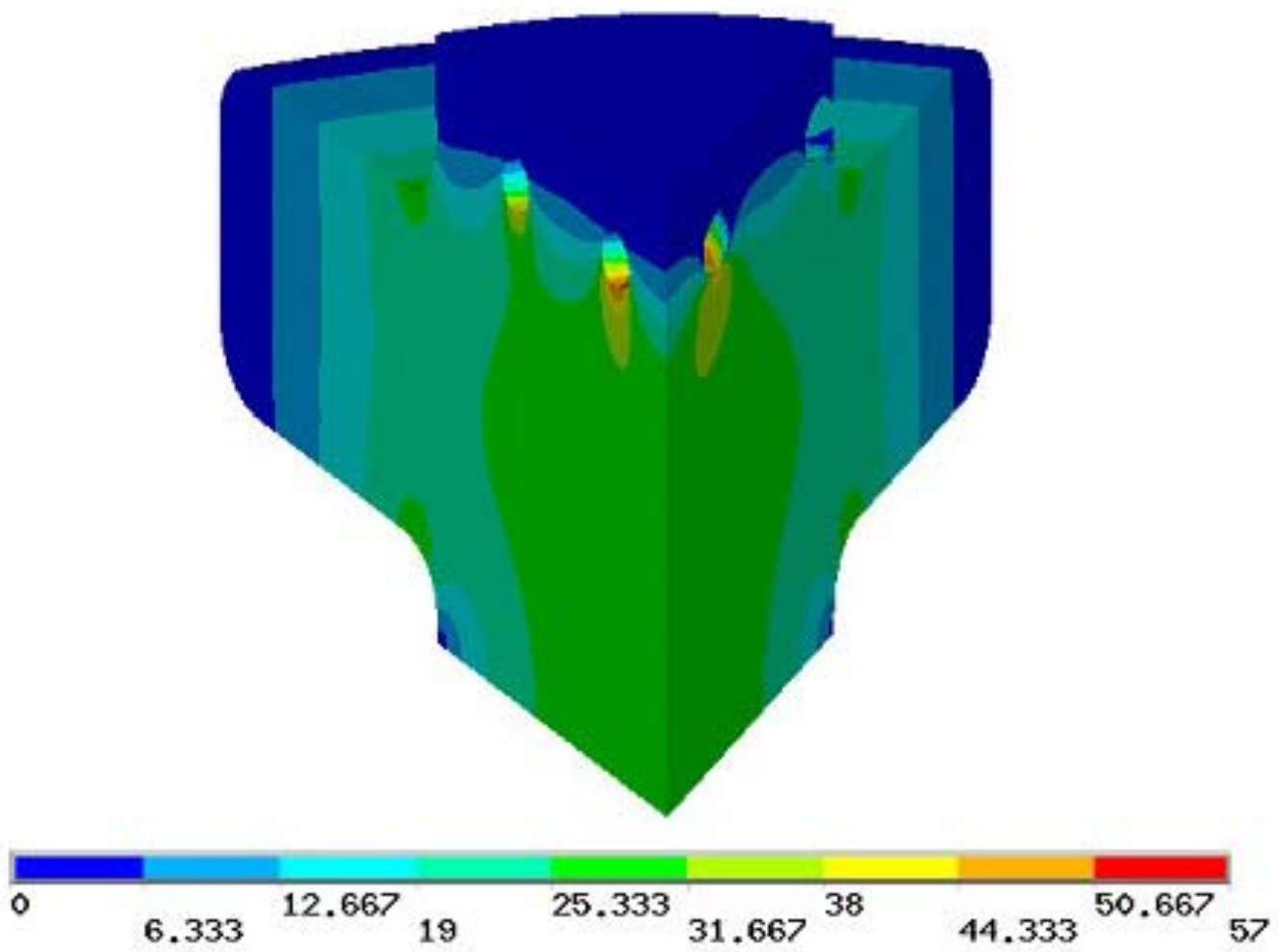

Figure 5.6. Contour Plot of the Electric Field Distribution in the Test Cavity Closed by the Baseline Gridded-Tube Window $(\mathrm{MV} / \mathrm{m})$. On-Axis Electric Field $=30$ $\mathrm{MV} / \mathrm{m}$.

Table 5.1. Effects of Both Grid Pattern and Tube Outer Diameter on the Surface Electric Field Enhancement, $\zeta$.

\begin{tabular}{ccc}
\hline \hline Pattern & Tube DIA=3/8" & Tube DIA=9/16" \\
\hline $4 \times 4$ Grid of touching tubes & 1.95 & 1.90 \\
$4 \times 4$ "waffle" grid & 1.90 & 1.75 \\
$6 \times 6$ "waffle" grid & 1.65 & 1.52 \\
$8 \times 8$ "waffle" grid & 1.42 & 1.31 \\
\hline
\end{tabular}


Table 5.2. Geometrical Parameters of the Baseline Gridded-Tube Window.

\begin{tabular}{cc}
\hline \hline & Parameter \\
\hline Grid radius $(\mathrm{mm})$ & 80.01 \\
Grid pattern & $4 \times 4$, "waffle" \\
Grid spacing (c-c) $(\mathrm{mm})$ & 34.93 \\
Tube outer diameter $(\mathrm{mm})$ & 9.53 \\
\hline
\end{tabular}

Table 5.3. Finite Element Results for the $805 \mathrm{MHz}$ Test Cavity with the Baseline Gridded-Tube Window.

\begin{tabular}{cc}
\hline \hline Parameter & FEA Value \\
\hline Frequency $(\mathrm{MHz})$ & 803.448 \\
Quality factor & 18191.205 \\
Maximum field enhancement- grid & 1.90 \\
Maximum heat flux- grid $\left(\mathrm{W} / \mathrm{m}^{2}\right)$ & 9586.022 \\
Maximum heat flux- cavity $\left(\mathrm{W} / \mathrm{m}^{2}\right)$ & 4904.6 \\
Maximum heat flux- Be window $\left(\mathrm{W} / \mathrm{m}^{2}\right)$ & 1942.066 \\
\hline
\end{tabular}

\subsection{Thermal Analysis}

The thermal finite element mesh was constructed at the interface between the vacuum and the grid. The power loss density results were transferred from the electromagnetic mesh to the thermal mesh. The thermal mesh is shown in Figure 5.5 where 3-node shell elements were employed. The relative minimum thermal element size with respect to the inner radius of the cavity, $r_{e} / b$, equals about 0.002 , and the total number of thermal elements is about 39,000. The finite element solution for the power loss density is shown in Figures 5.7 and 5.8. It is apparent that only the internal side of the grid was subjected to high power loss density, whereas the external side of the grid was subjected to negligible power loss density. The boundary conditions for the grid include power loss density (obtained from the electromagnetic 
analysis $)$ and a reference temperature $\left(0^{\circ} \mathrm{C}\right)$ specified at the edge of the gridded-tube window. Figures 5.9 and 5.10 show the contour plots of the temperature distribution for the baseline gridded-tube window with a grid wall thickness of $254 \mu \mathrm{m}$. There is small variation in the temperature between the internal side and the external side. The maximum temperature was about $55.6^{\circ} \mathrm{C}$ located at the middle of the grid. The temperature profile is quantitatively and qualitatively similar to the that obtained in the beryllium window considered in Chapter 4. The thermal finite element results are summarized in Table 5.3. Figure 5.11 shows the transient profile of the maximum temperature. The maximum steady-state temperature was obtained after about 220 seconds. Figure 5.12 shows the dependence of the temperature on the grid wall thickness in the baseline gridded-tube window.

At the beginning of the study, it is assumed that cooling of the tubes by helium gas would be necessary to reduce the temperature rise in the grid. Helium gas was chosen to be the cooling candidate due to its large radiation length and cooling capabilities. A forced convection study was performed to obtain the heat transfer coefficient as a function of the mass flow rate. For the present calculations, assuming fully developed turbulent flow, a practical heat transfer coefficient of $250 \mathrm{~W} / \mathrm{m}^{2} \cdot \mathrm{K}$ was assumed. This would correspond to a mass flow rate of about $7.9 \times 10^{-4} \mathrm{~kg} / \mathrm{s}$. Figures 5.13 and 5.14 show the contour plots of the temperature distribution for the gas-cooled baseline gridded-tube window with a grid wall thickness of $254 \mu \mathrm{m}$. The maximum temperature is about $7.7^{\circ} \mathrm{C}$ located at the internal surface of the grid, and shifted from the middle of the grid. The temperature varies around the tubes in the grid. Figures 5.15 and 5.16 show the contour plots of the temperature distribution for the gas-cooled baseline gridded-tube window with a grid wall thickness of $100 \mu \mathrm{m}$. For this case, a significant difference in the temperature was obtained between the external side and the internal side. Figure 5.17 shows the dependence of the temperature on the grid wall thickness in the baseline gridded-tube window with cooling. 

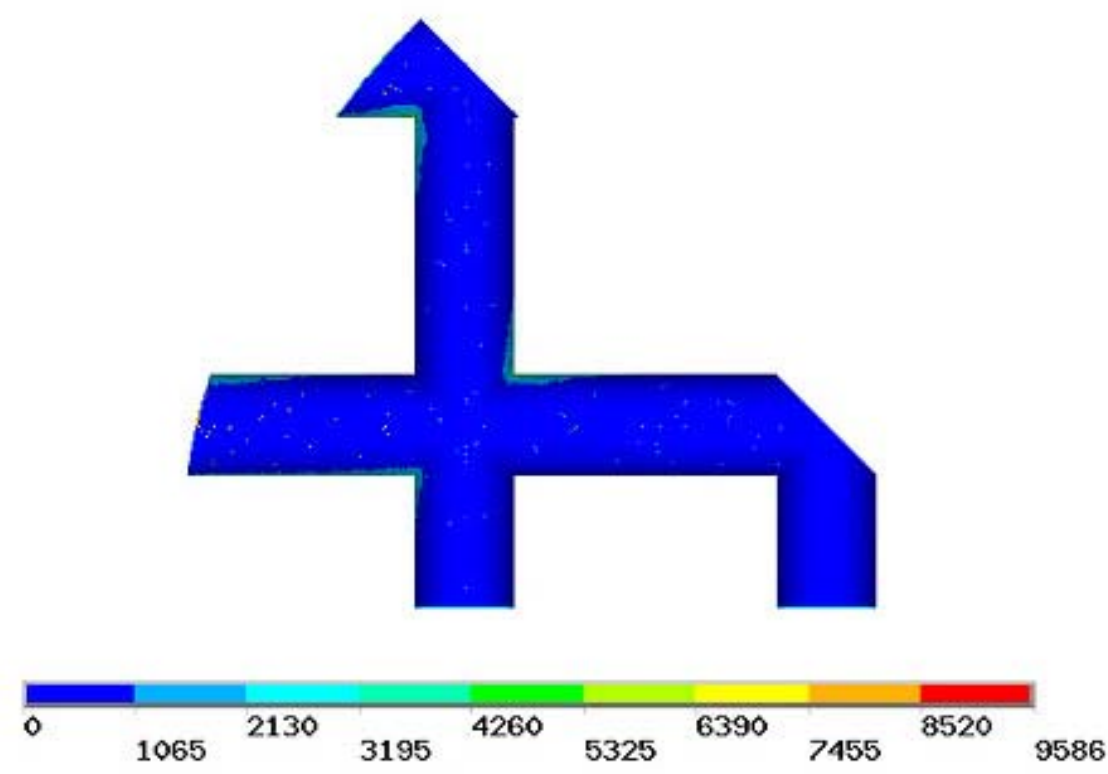

Figure 5.7. Contour Plot of the Power Loss Density in the Baseline Gridded-Tube Window $\left(\mathrm{W} / \mathrm{m}^{2}\right)$ (External Side).

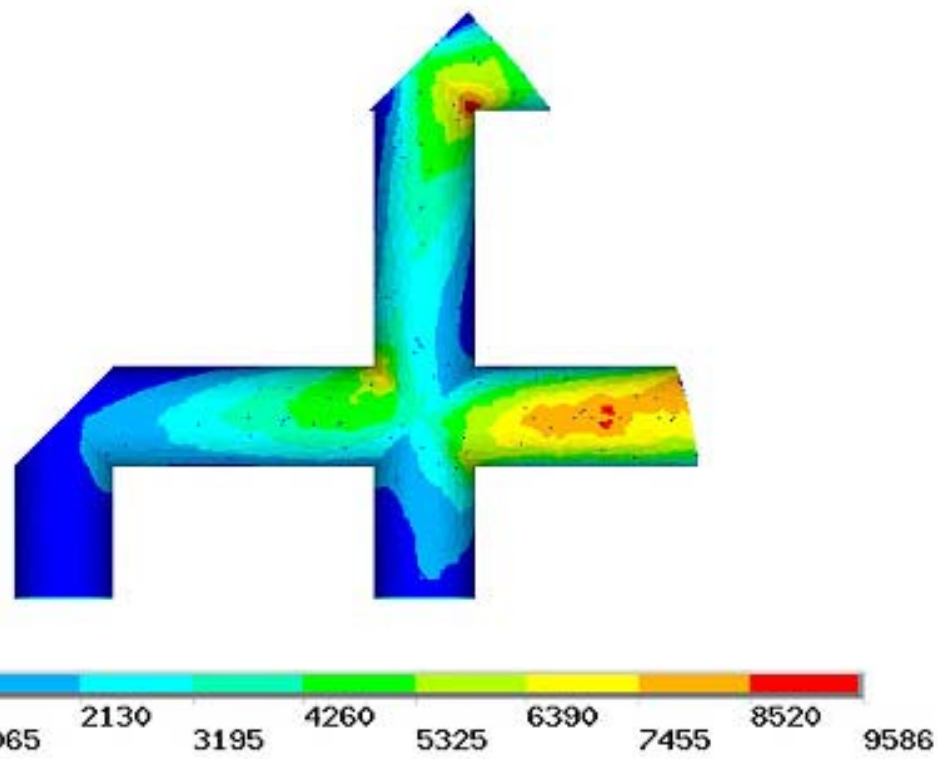

Figure 5.8. Contour Plot of the Power Loss Density in the Baseline Gridded-Tube Window $\left(\mathrm{W} / \mathrm{m}^{2}\right)$ (Internal Side). 

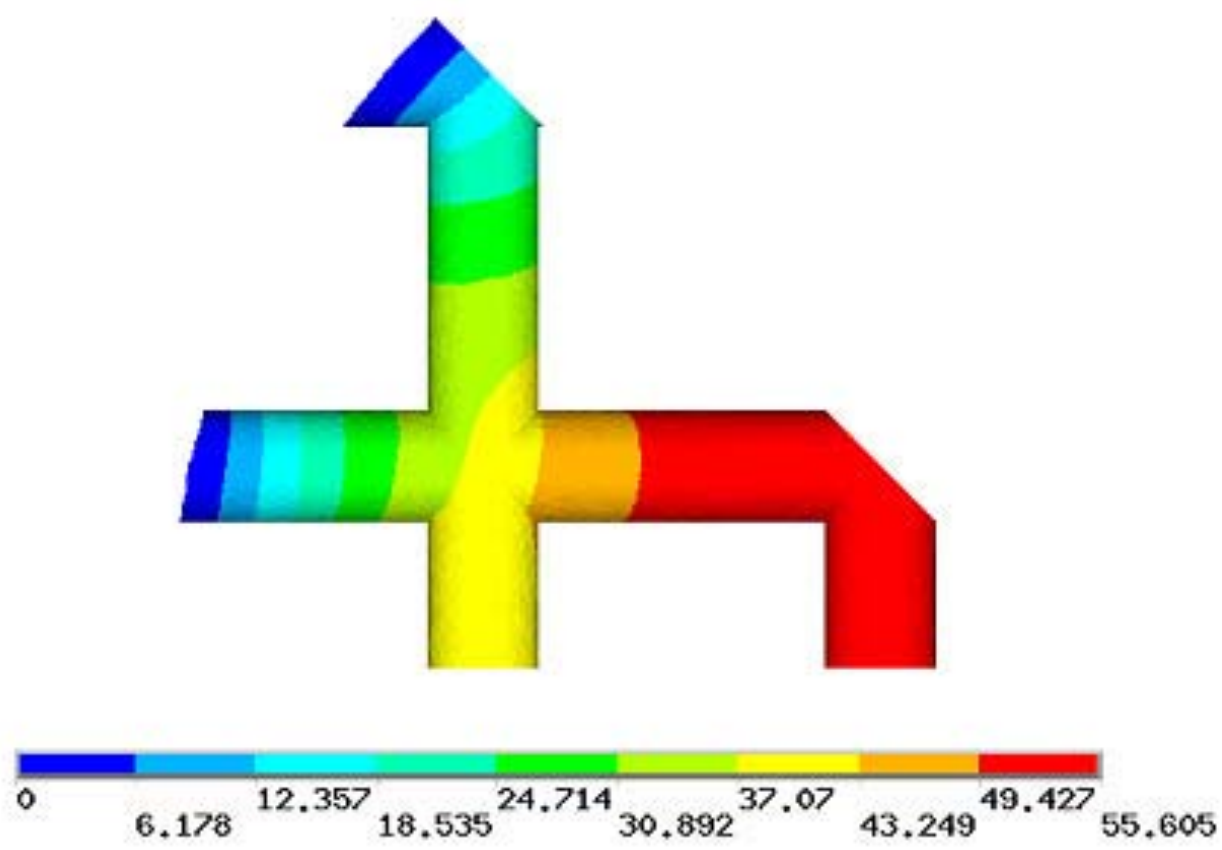

Figure 5.9. Contour Plot of the Temperature Distribution in the Non-Cooled Baseline Gridded-Tube Window $\left({ }^{\circ} \mathrm{C}\right)$. Grid Wall Thickness $=254 \mu \mathrm{m}$. (External Side).
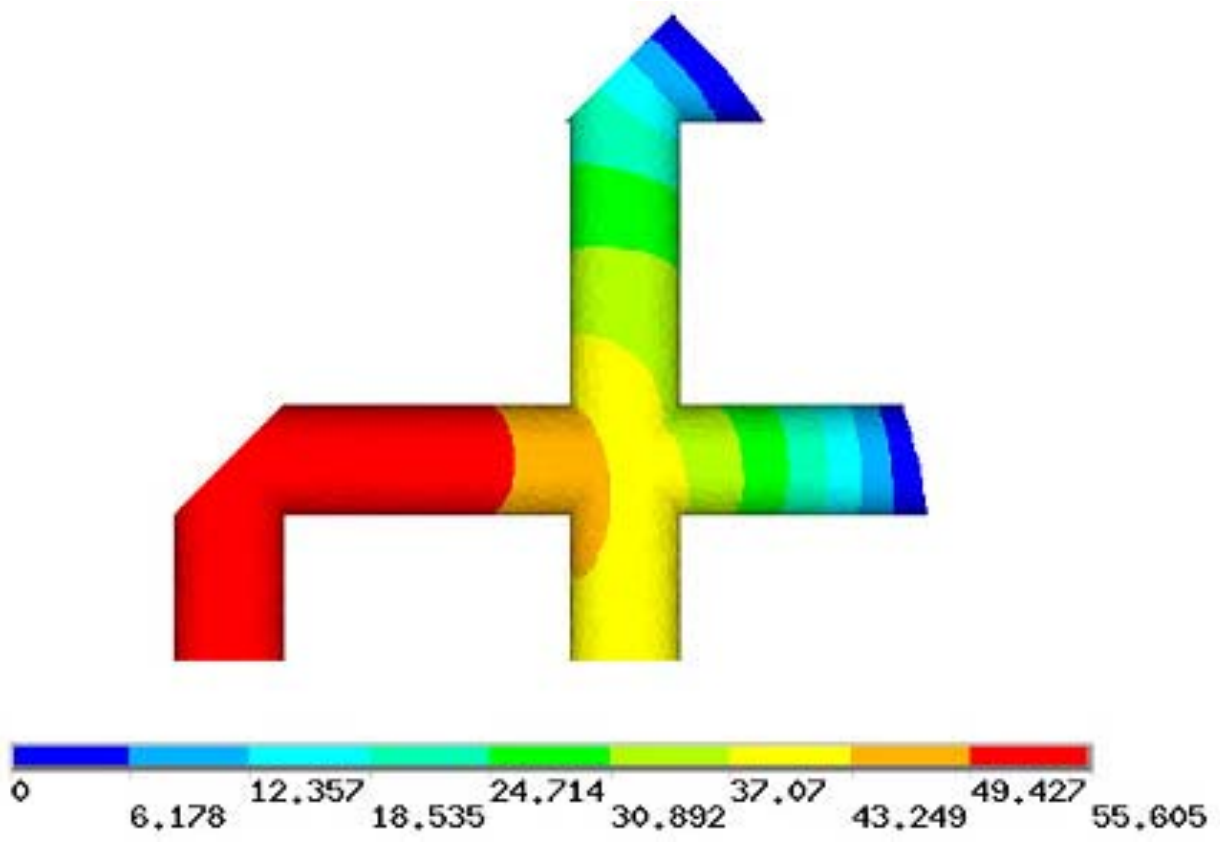

Figure 5.10. Contour Plot of the Temperature Distribution in the Non-Cooled Baseline Gridded-Tube Window $\left({ }^{\circ} \mathrm{C}\right)$. Grid Wall Thickness $=254 \mu \mathrm{m}$. (Internal Side). 


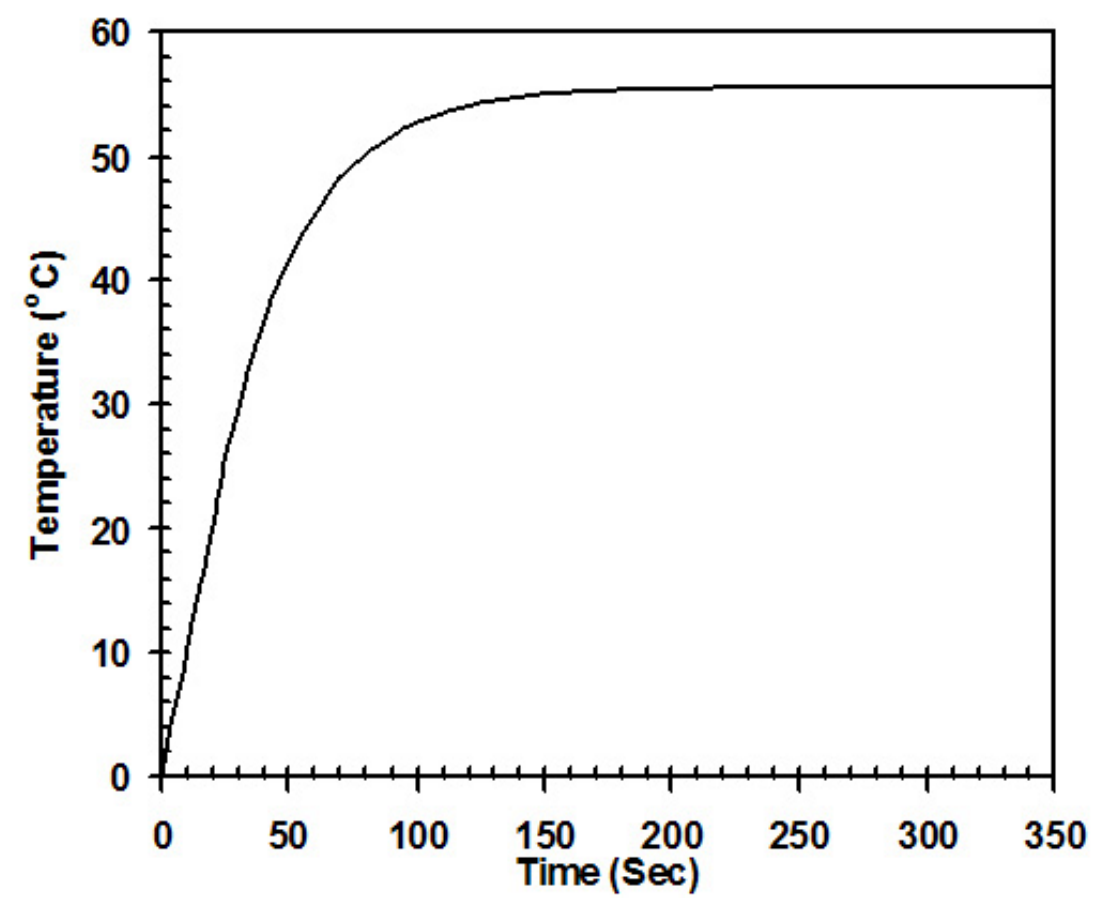

Figure 5.11. The Transient Maximum Temperature Profile of the Non-Cooled Baseline Gridded-Tube Window. Grid Wall Thickness $=254 \mu \mathrm{m}$.

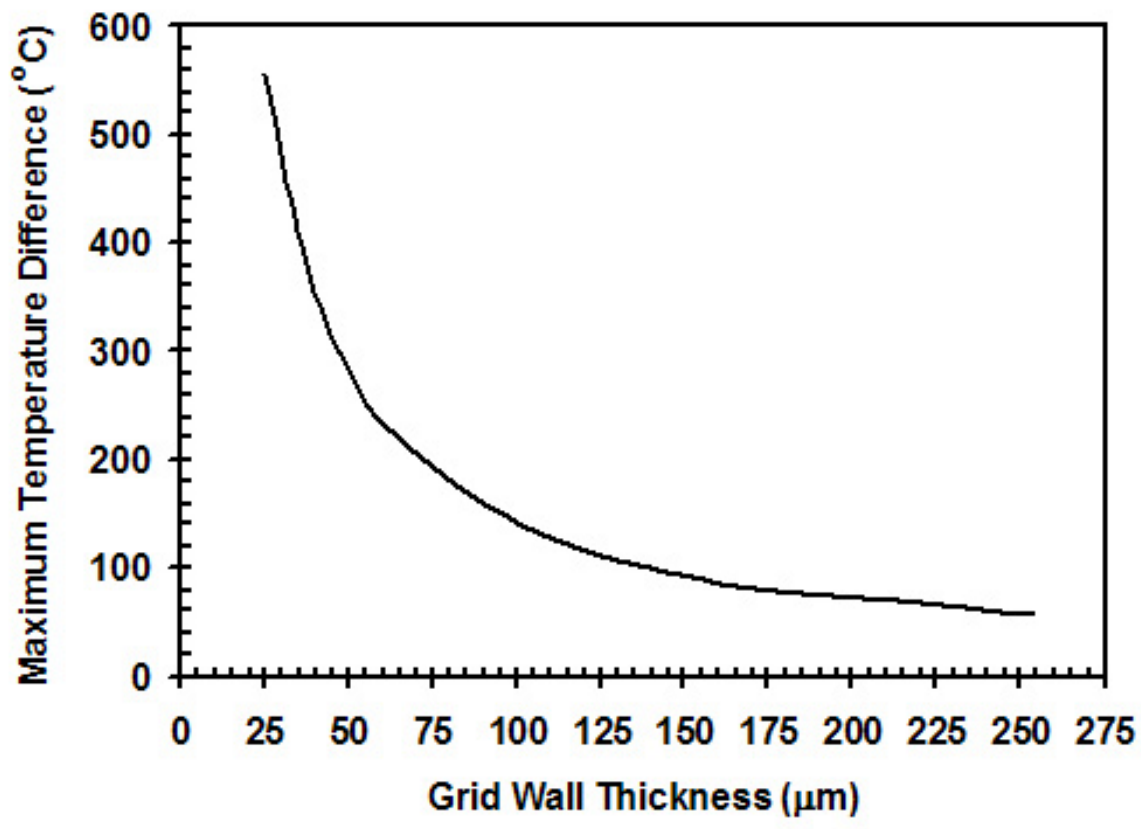

Figure 5.12. Dependence of the Temperature Profile on the Grid Wall Thickness for the Non-Cooled Baseline Gridded-Tube Window. 


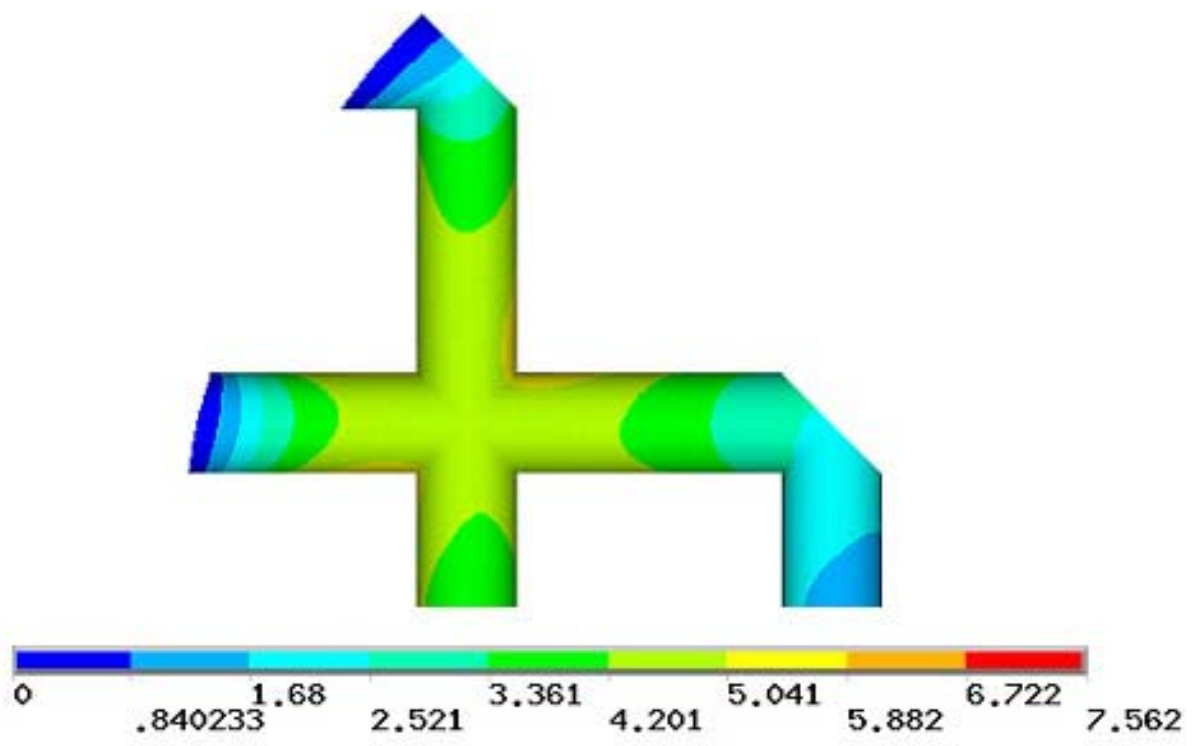

Figure 5.13. Contour Plot of the Temperature Distribution in the Gas-Cooled Baseline Gridded-Tube Window $\left({ }^{\circ} \mathrm{C}\right)$. Grid Wall Thickness $=254 \mu \mathrm{m}$. (External Side).
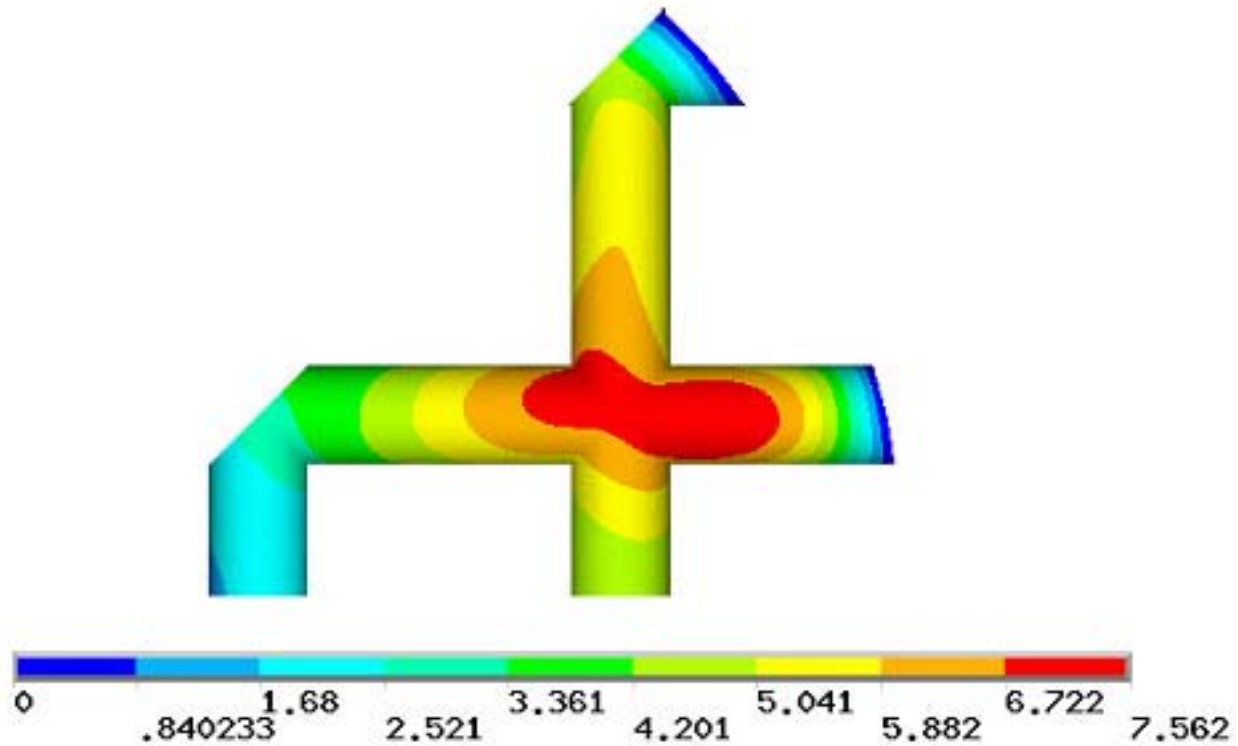

Figure 5.14. Contour plot of the Temperature Distribution in the Gas-Cooled Baseline Gridded-Tube Window $\left({ }^{\circ} \mathrm{C}\right)$. Grid Wall Thickness $=254 \mu \mathrm{m}$. (Internal Side). 


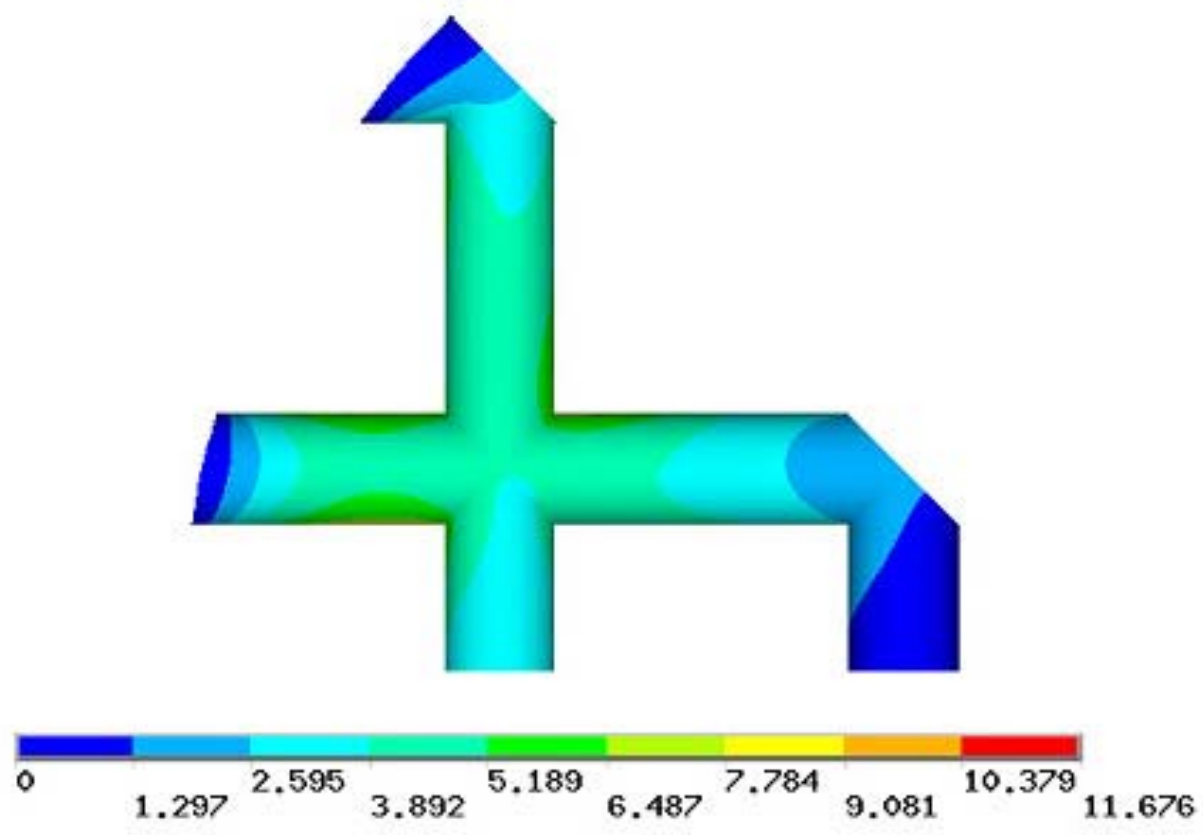

Figure 5.15. Contour Plot of the Temperature Distribution in the Gas-Cooled Baseline Gridded-Tube Window $\left({ }^{\circ} \mathrm{C}\right)$. Grid Wall Thickness $=100 \mu \mathrm{m}$. (External Side).

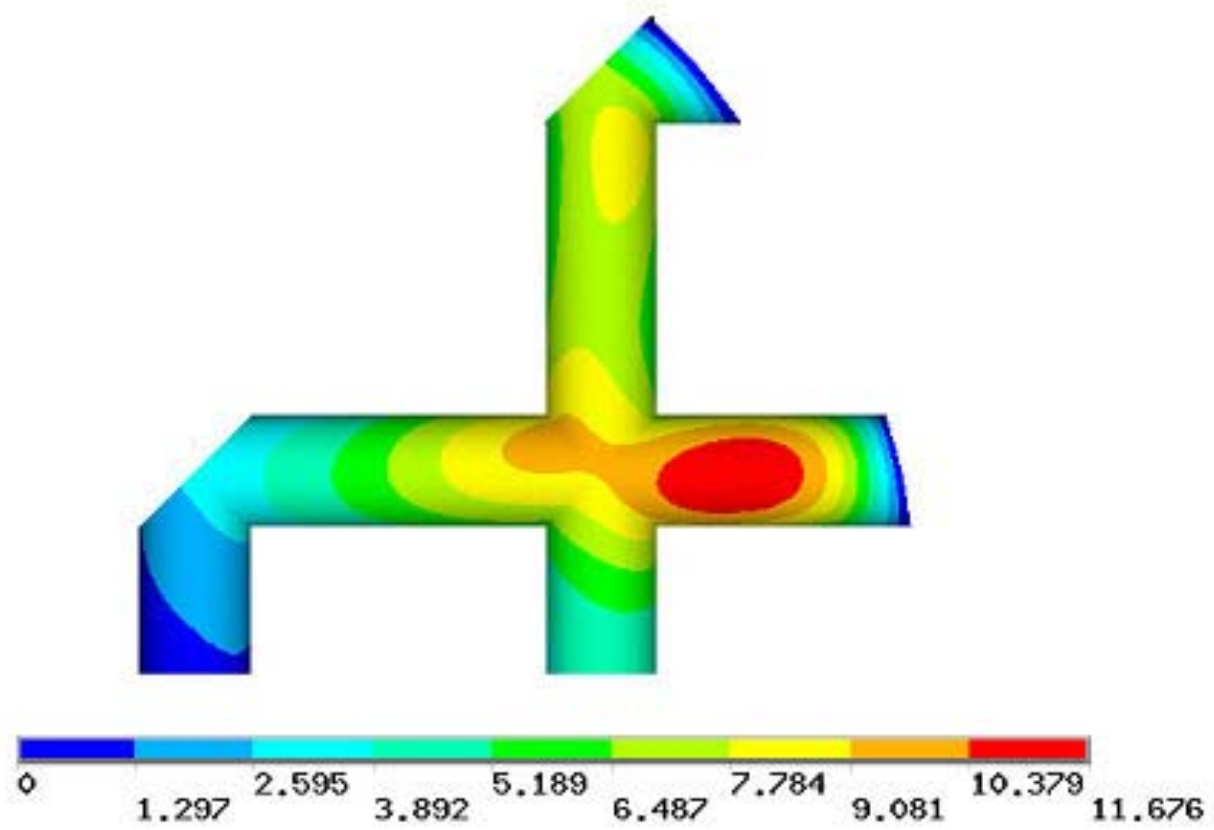

Figure 5.16. Contour Plot of the Temperature Distribution in the Gas-Cooled Baseline Gridded-Tube Window $\left({ }^{\circ} \mathrm{C}\right)$. Grid Wall Thickness $=100 \mu \mathrm{m}$. (Internal Side). 


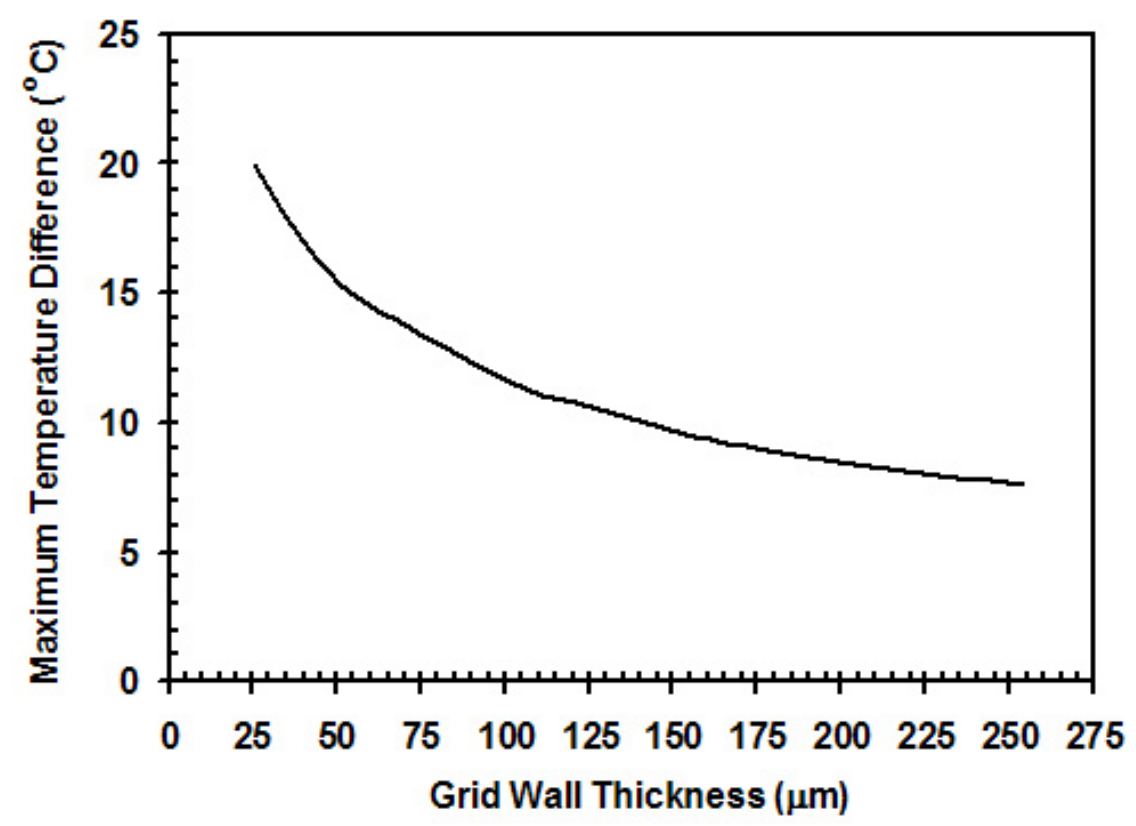

Figure 5.17. Dependence of the Temperature Profile on the Grid Wall Thickness for the Gas-Cooled Baseline Gridded-Tube Window. $\left(h=250 \mathrm{~W} / \mathrm{m}^{2} \mathrm{~K}\right)$.

\subsection{Structural Analysis}

The temperature profile was transferred from the thermal mesh to the structural mesh. A structural analysis was then performed to solve for the displacements and the thermal stresses. The edge of the grid was assumed to be completely clamped to the cavity (rotations and displacements at the nodes laying on the edge of the grid were fixed).

Modeling the grids with solid continuum elements was impractical because of the very small thickness of the grid wall $254 \mu \mathrm{m}$. More 500,000 solid continuum elements would have been required to obtain mesh-independent stress results. The stress was expected to be high at the joints of the tubes. So a very fine mesh was used in these region. Figure 5.18 shows the structural finite element mesh created with 8-node shell elements. Here, the relative minimum electromagnetic element size 
with respect to the inner radius of the cavity, $r_{e} / b$, equals about 0.002 , and the total number of elements is about 39,000.

Structural analysis of gridded-tube structures requires a mesh convergence study, in which various finite element models, each with a different mesh density, are investigated separately. Figure 5.19 shows the dependence of the maximum von Mises stress on the number of elements. a fixed element size was used in all the regions in order to understand the effect of number of elements on the maximum von Mises stress. It was found that a mesh with fine elements at the intersectional regions gives more accurate results than a mesh with a higher total number of elements but coarser in the intersectional regions. It is observed that the maximum von Mises stress decreases with increasing number of elements. This continues until a mesh independent stress value is obtained. The displacement results were found to be constant for all the finite element models used to generate Figure 5.19.

$\mathrm{RF}$ heating can cause the windows to deform in the axial direction of the cavity. The resulting thermal stresses in the window must be maintained below the yield stress of the window material. The out-of-plane deflection must also be kept below 25 microns to prevent the frequency of the cavity from shifting more than $10 \mathrm{kHz}$. Consider the non-cooled baseline gridded-tube window with a tube wall thickness of $254 \mu \mathrm{m}$. Figure 5.20 shows the finite element displacement results. As expected, the displacement is much smaller than that for the beryllium windows due to the structural rigidity of the grids. The external side of the grid displaces toward the positive vertical direction, whereas the lower half of the grid displaces toward the negative vertical direction. This is due to the thermal expansion of the tubes in the grid. The displacement is higher at the joints of the tubes due to the thermal expansion of the four tubes at each joint. The maximum displacement, occurring at the middle of the grid, is about $110 \mu \mathrm{m}$. Figure 5.21 shows the von Mises stress 
result. The maximum von Mises stress is about $245 \mathrm{MPa}$. The yield stress for the grid material is about $275 \mathrm{MPa}$. So the yield stress safety factor is about 1.12 .

Consider the cooled baseline gridded-tube window with a grid wall thickness of $254 \mu \mathrm{m}$. The maximum displacement is reduced to $13 \mu \mathrm{m}$, and the maximum stress is reduced to $30.8 \mathrm{MPa}$. The yield stress safety factor is thus increased to 8.96. Figures 5.22 and 5.23 show the displacement results, and Figures 5.24 and 5.25 show the thermal stress results. If an array of parallel tubes that are free to expand was considered instead, there would have been negligible out-of-plane deflection, and very low thermal stresses. But unfortunately, this geometry doesn't effectively terminate the fields.

The fundamental natural frequency for a clamped circular plate is given by:

$$
F_{0}=5.11\left[\frac{E t^{3}}{12 \pi \bar{a}^{2} m\left(1-\nu^{2}\right)}\right]^{1 / 2}
$$

where $\bar{a}$ is the radius of the plate, $E$ is the modulus of elasticity of the material, $\nu$ is the poisson's ratio of the material, $t$ is the thickness of the plate, and $m$ is the mass of the plate. [see e.g., Schiff (1990)]. The fundamental natural frequency was calculated for both the beryllium window and an aluminum window that has the same geometry as the beryllium window using the previous formula. The finite element solutions of the frequency were obtained using axisymmetric shell elements. Precise agreement was obtained between the finite element and the analytical results as shown in Table. 7.1. A finite element analysis of the baseline gridded-tube window with a grid wall thickness of $254 \mu \mathrm{m}$ was performed using 8-node shell elements. The fundamental natural frequency results are given in Table. 5.4. Due to the structural integrity of the grid compared to that of the beryllium foils, the fundamental natural frequency of the grid is higher than that of the foil. The mode shape associated with the grid fundamental natural frequency is shown in Figure 5.26. 


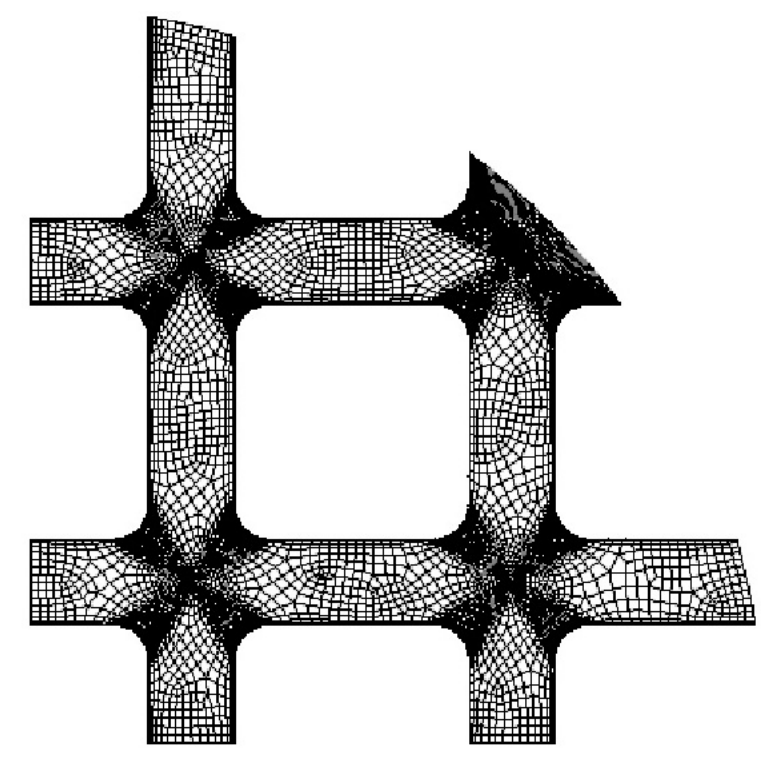

Figure 5.18. Finite Element Structural Mesh of the Baseline Gridded-Tube Window (Relative Minimum Element Size with Respect to the Inner Radius of the Cavity, $r_{e} / b$, Equals About 0.002).

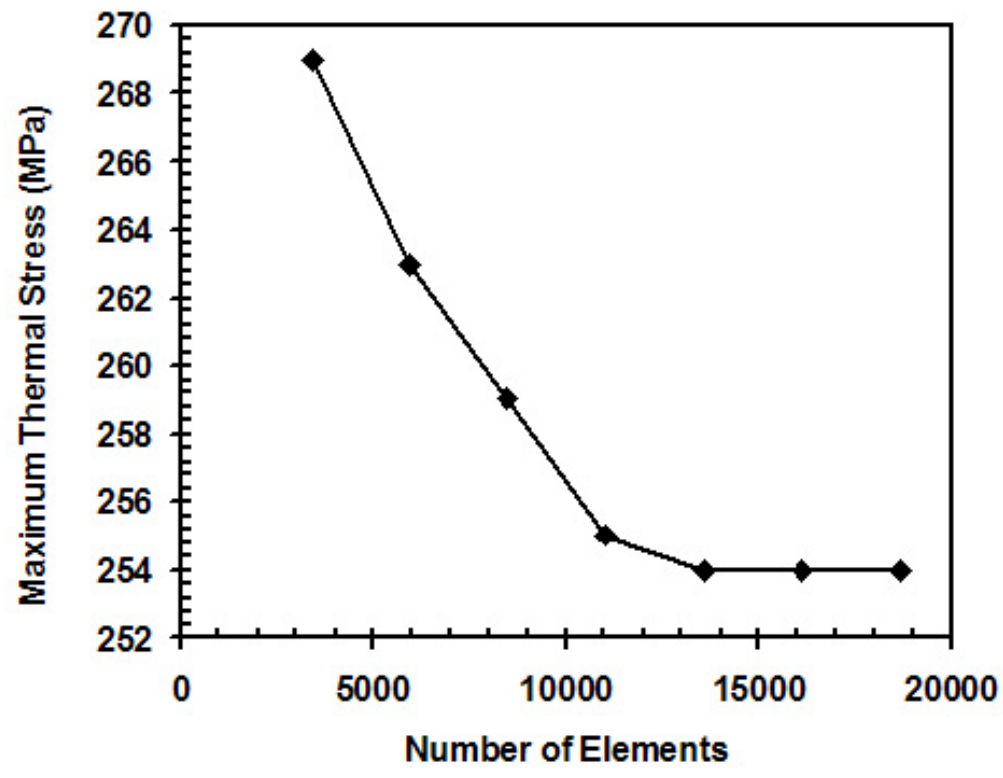

Figure 5.19. Dependence of the Numerical Value of the Thermal Stress on the Number of Elements for the Baseline Gridded-Tube Window. Grid Wall Thickness $=254 \mu \mathrm{m}$ (Uniform Meshing). 

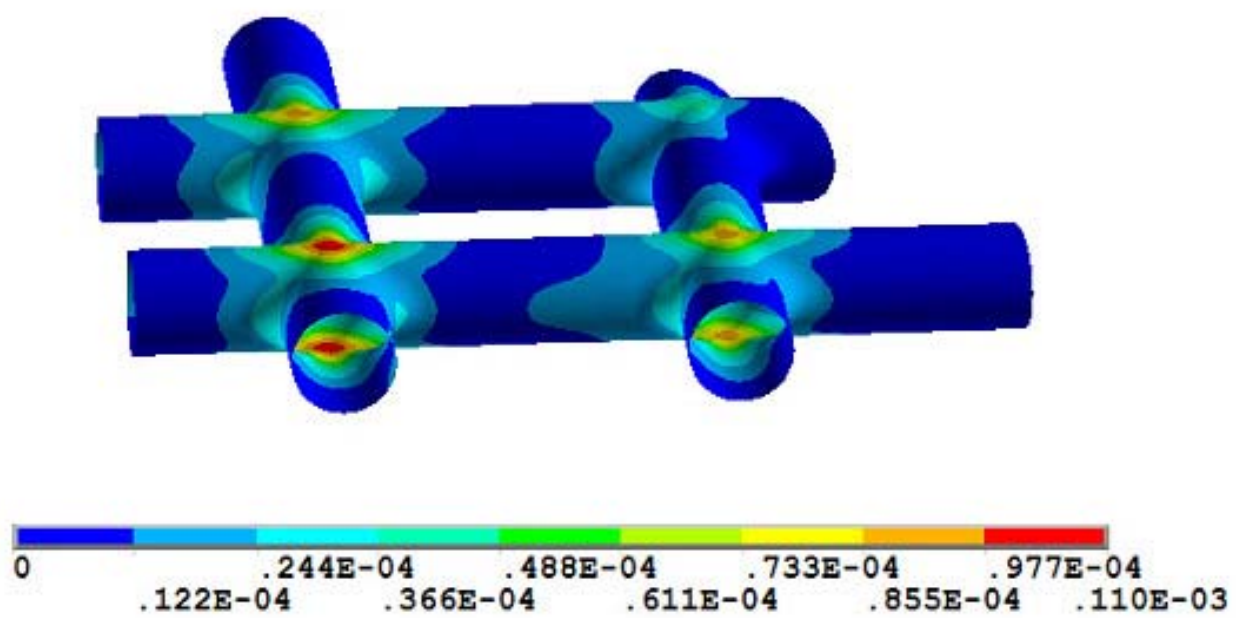

Figure 5.20. Contour Plot of the Total Displacement of the Non-Cooled Baseline Gridded-Tube Window, $(m)$, Grid Wall Thickness $=254 \mu \mathrm{m}$ (Internal Side).
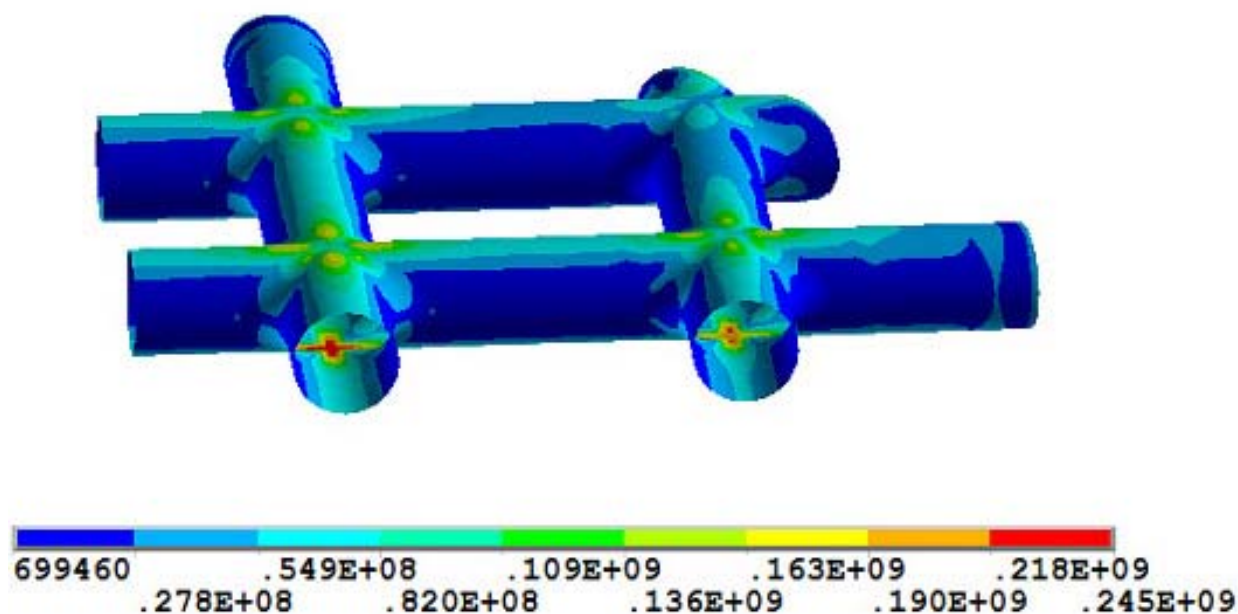

Figure 5.21. Contour Plot of the von Mises Stress in the Non-Cooled Baseline Gridded-Tube Window (Pa). Grid Wall Thickness $=254 \mu \mathrm{m}$. 


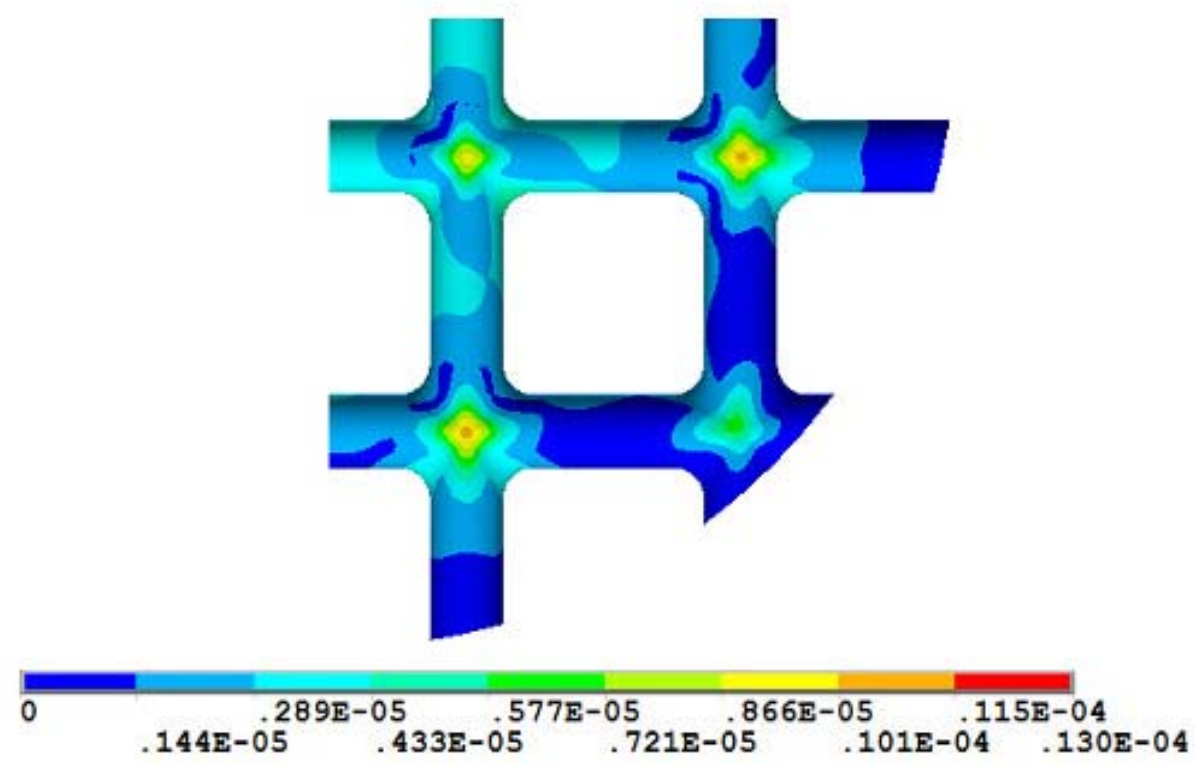

Figure 5.22. Contour Plot of the Total Displacement of the Gas-Cooled Baseline Gridded-Tube Window $(\mathrm{m})$. Grid Wall Thickness $=254 \mu \mathrm{m}$ (External Side).

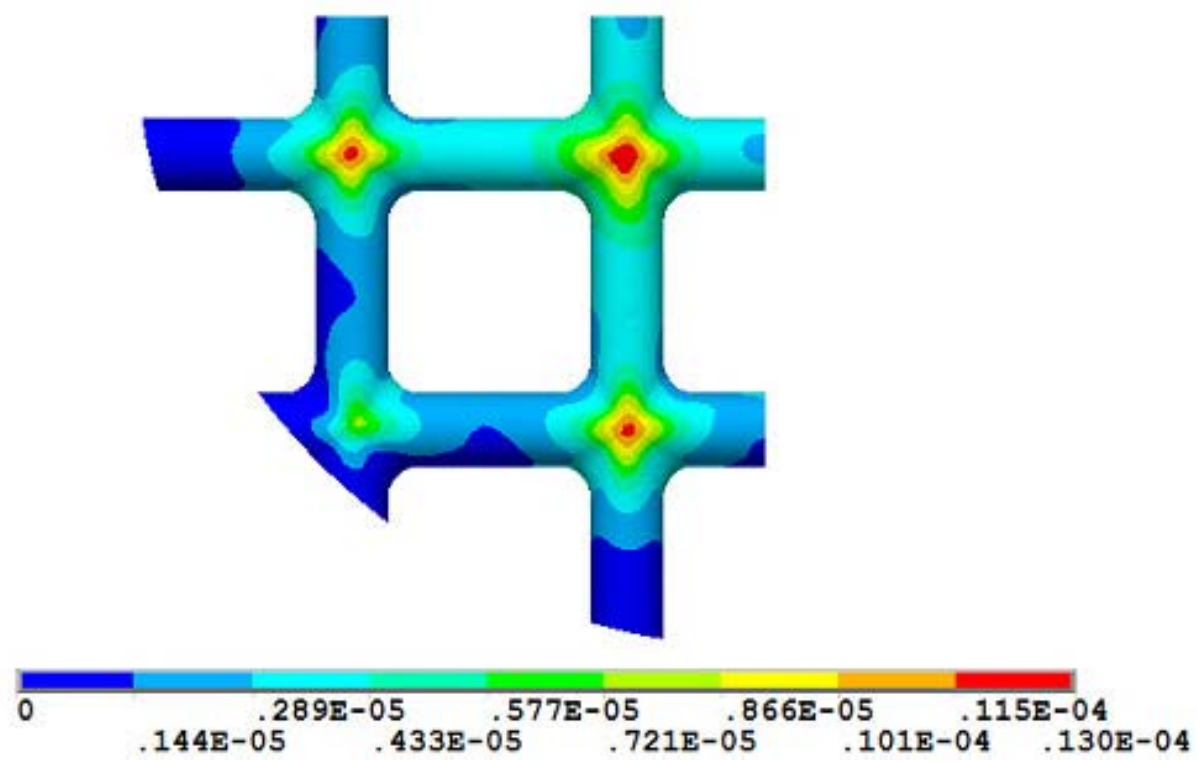

Figure 5.23. Contour Plot of the Total Displacement of the Gas-Cooled Baseline Gridded-Tube Window (m). Grid Wall Thickness $=254 \mu \mathrm{m}$ (Internal Side). 


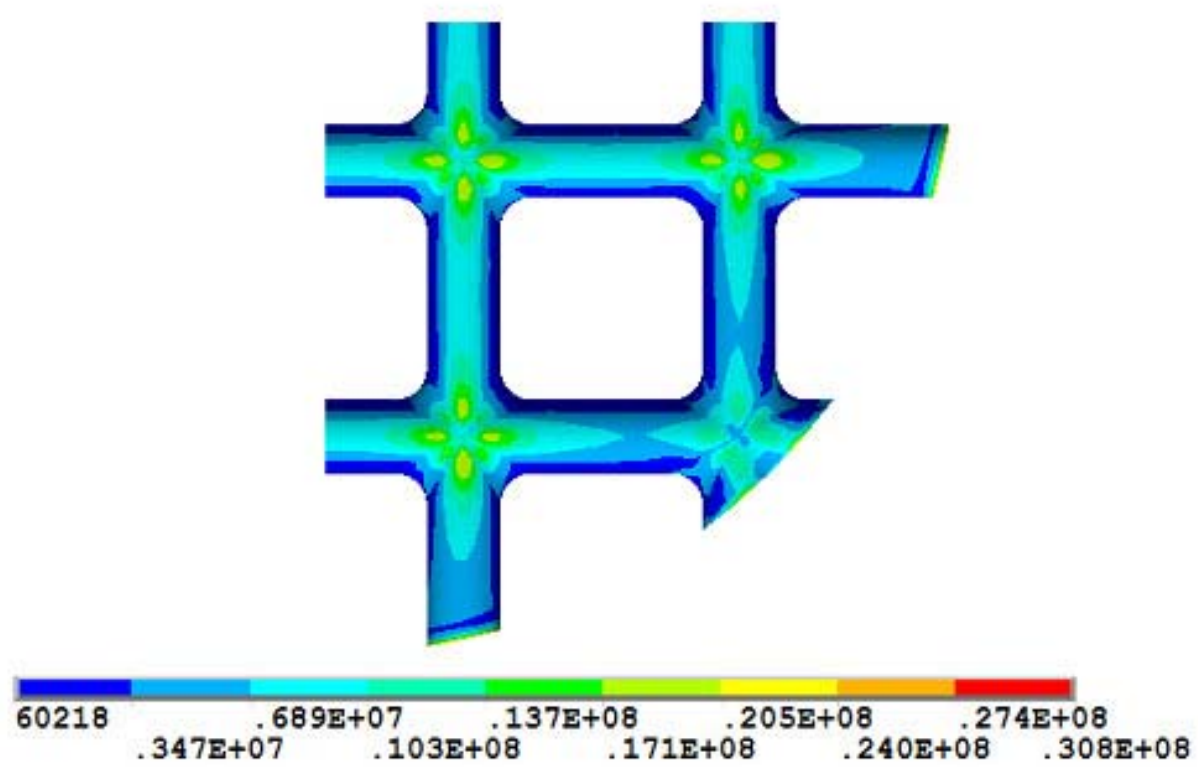

Figure 5.24. Contour Plot of the von Mises Stress in the Gas-Cooled Baseline GriddedTube Window $(\mathrm{m})$. Grid Wall Thickness $=254 \mu \mathrm{m}$ (External Side).
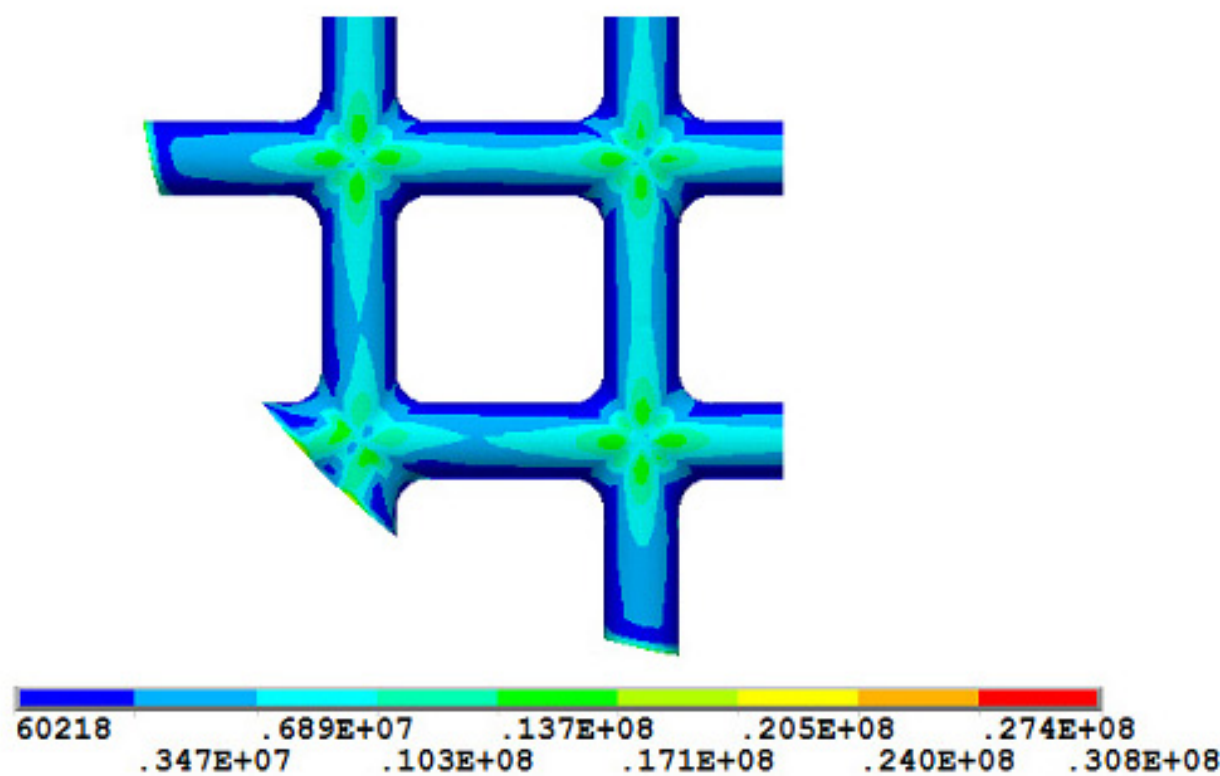

Figure 5.25. Contour Plot of the von Mises Stress in the Gas-Cooled Baseline GriddedTube Window $(\mathrm{Pa})$. Grid Wall Thickness $=254 \mu \mathrm{m}$ (Internal Side). 


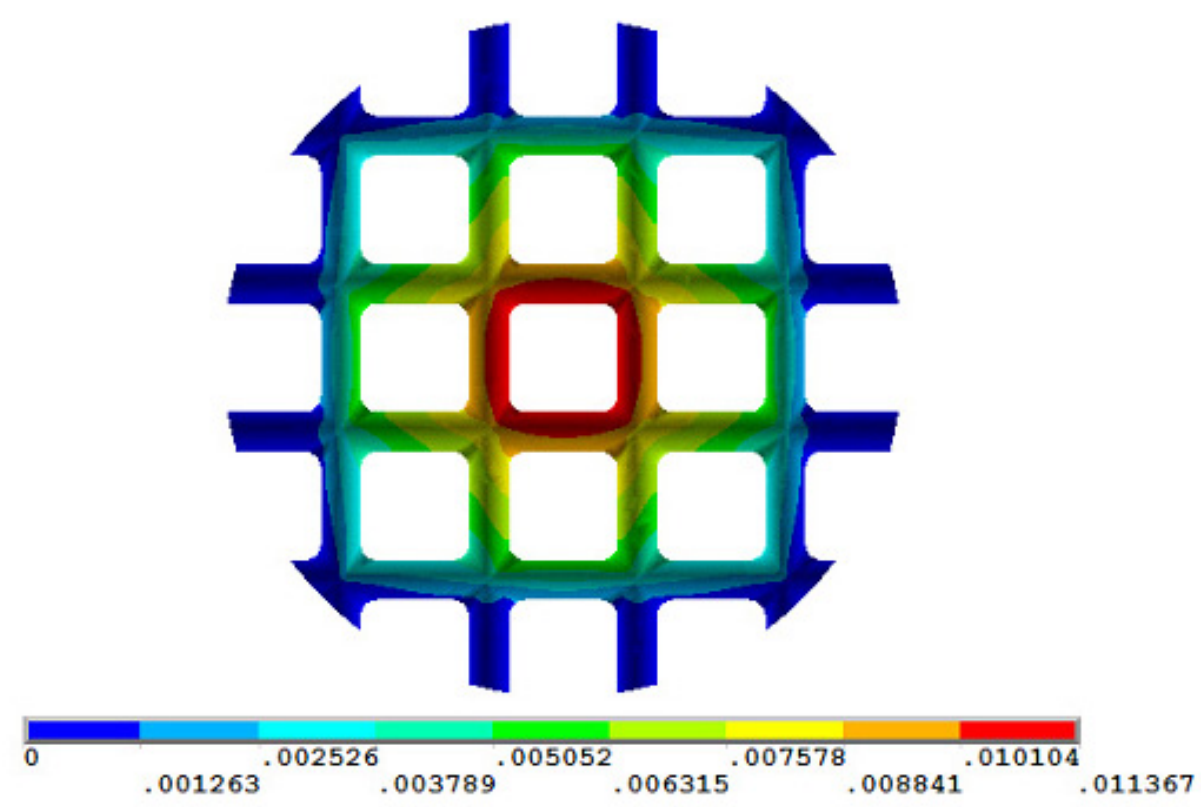

Figure 5.26. Mode Shape Associated With the Fundamental Natural Frequency of the Baseline Gridded-Tube Window. Grid Wall Thickness $=254 \mu \mathrm{m}$.

Table 5.4. Analytical and Finite Element Solutions of the Fundamental Natural Frequency of Various RF Cavity Windows.

\begin{tabular}{ccc}
\hline \hline Window Type & FEA Value & Analytical Value \\
\hline Aluminum 6061-T6 foil, $(t=127 \mu \mathrm{m})$ & 49.89 & 49.87 \\
Beryllium IF1 foil, $(t=127 \mu \mathrm{m})$ & 120.35 & 120.33 \\
Aluminum 6061-T6 baseline grid, $(t=254 \mu \mathrm{m})$ & 1969.9 & - \\
\hline
\end{tabular}




\section{CHAPTER 6 \\ EXPERIMENTAL AND FINITE ELEMENT STUDIES OF THE PERFORMANCE OF THE TEST CAVITY}

In section 6.1, finite element and experimental studies of the deflection of the test cavity windows are presented. In section 6.2 , the electromagnetic behavior of the test cavity under RF power operation is demonstrated and the field emission results are described. The experimental results for the frequency, quality factor, and surface electric field enhancement are compared to corresponding finite element results.

\subsection{Deflection of the RF Cavity Beryllium Windows}

The beryllium windows are circular, flat foils with a diameter of $16 \mathrm{~cm}$, and a thickness of $127 \mu \mathrm{m}$. They undergo significant ohmic heating due to RF power, and displace out-of-plane. The deformation of the beryllium windows subjected to thermal loading was studied by [Hartman et al. (2000), Hartman et al. (2001)]. In that study, two experimental setups were used. In the first experimental setup, a stress free aluminum window was heated with a halogen bulb in a temperature-controlled enclosure. It was found that the temperature profile in the window due to the halogen bulb heating was similar to that due to the RF power. Temperature was measured with an infrared camera, and displacement was measured as function of foil radius with a dial gauge. In the second experimental setup, a pre-stressed beryllium window was mounted inside a prototype RF cavity. Temperature in the window was measured with thermocouples, and displacement was measured with a dial gauge mounted in the center of the window.

In this work, the aluminum window used in the first experimental setup was modeled to obtain the out-of-plane displacement. Axisymmetric shell elements were used. To determine the direction of bowing, a small curved perturbation in the window geometry was implemented. But, the displacement was found to be dependent 
on the magnitude of the perturbation, which indicated inaccurate results. To overcome this problem, another technique was used, where a small force was first applied toward the desired direction of bowing at the center of the window. Then, the temperature profile was applied and the applied force was removed. Using this technique, the displacement results were found to be independent of the magnitude of the force. In Figure 6.1, the experimental displacement profile is shown by the solid line, and the finite element displacement profile is shown by the triangles (FEA1). It was found that applying a small initial displacement at the center of the window before applying the temperature loads could reduce the difference between the experimental and the finite element profiles. The finite element displacement profile with a $0.5 \mathrm{~mm}$ initial displacement, applied at the center of the window, is shown by the diamonds (FEA2) in Figure 6.1. Excellent agreement was obtained between the experimental displacement profile and FEA2. The application of the initial displacement in FEA2 may have taken into account the experimental effects including the weight of the dial gauge used to measure the center displacement.

To predict the out-of-plane displacement of the pre-stressed beryllium window used in the second experimental setup, a combined iterative finite elementexperimental procedure was constructed. First, a specific temperature decrease was applied to the window. Second, the RF temperature profile was applied. Third, the displacement was compared to the experimental results. Fourth, the specific temperature decrease was changed, and the finite element procedure was repeated until an excellent agreement between the experimental and the numerical profiles was obtained as shown in Figure 6.2. The pre-stress value was determined to be about 115 MPa. 


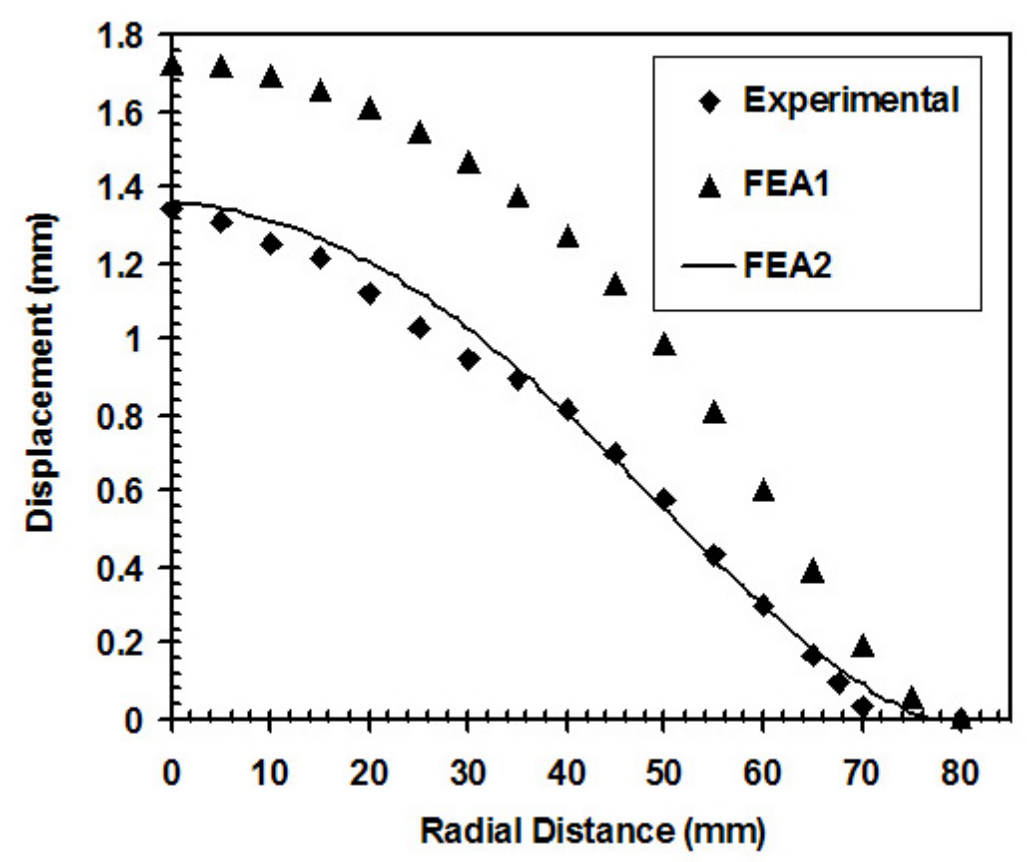

Figure 6.1. Finite Element and Experimental Results of the Out-Of-Plane Deflection of the Stress Free Aluminum Window

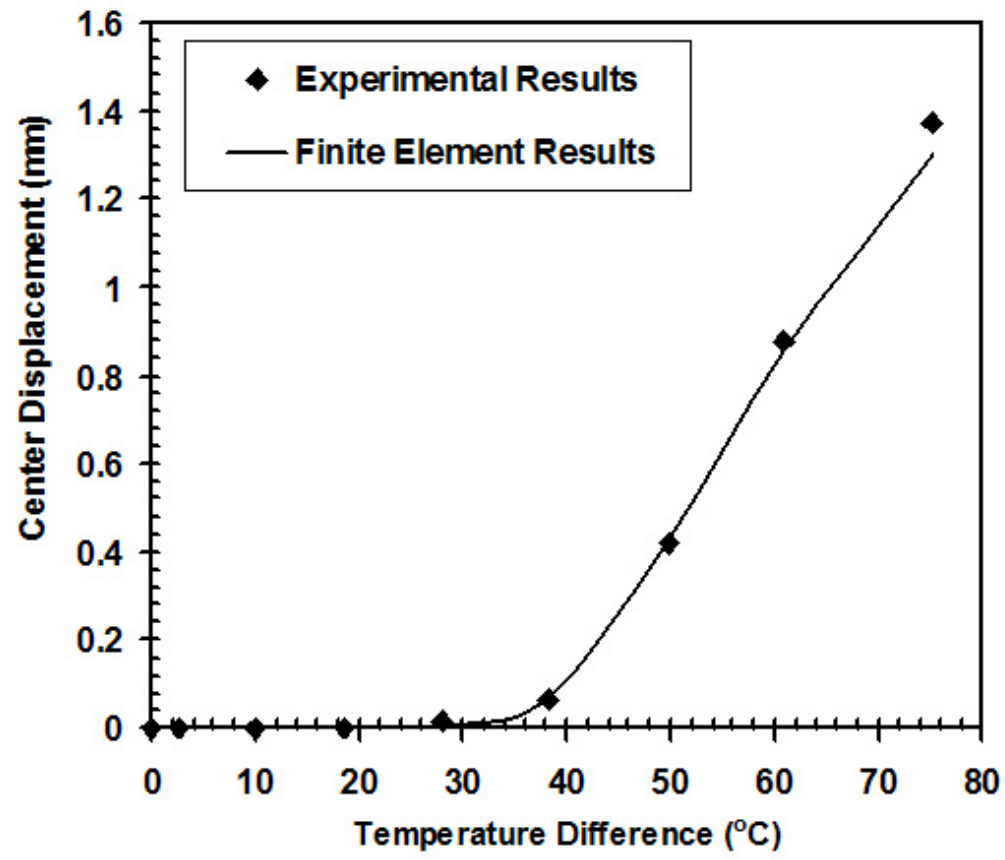

Figure 6.2. Finite Element and Experimental Results of the Out-Of-Plane Deflection of the Pre-Stressed Beryllium Window 


\subsection{Electromagnetic Analysis of the Cavity under RF Power Operation}

Recently, an $805 \mathrm{MHz}$ cavity with copper windows was built and subjected to high RF power [Li et al. (2002)a]. The on-axis electric field was reported to reach $34 \mathrm{MV} / \mathrm{M}$ (which is $13.3 \%$ higher than the theoretical design value) with negligible sparking rates. The low power measurement of the frequency of the cavity with copper windows was about $805.1 \mathrm{MHz}$. In the present work, the finite element frequency is about $808.3 \mathrm{MHz}$ for the $805 \mathrm{MHz}$ test cavity with beryllium windows. The difference is due to the geometrical differences between the cavity considered in the experiment and the cavity considered in this thesis. The finite element frequency for the cavity with the baseline grided-tube window is about 803.4. The difference from the experimental value is due to the effect of the grid geometry in the cavity, and the effect of the volume existing between the gridded-tube window and the titanium window.

The low power measurement of the quality factor of the cavity with copper windows was about 15, 080 as reported by [Li et al. (2002)a]. The theoretical design value of the quality factor is 18,800 . The present finite element results of the frequency and the quality factor are reported in Tables 4.1 and 5.3 for the cavity with the beryllium windows and the cavity with the baseline gridded-tube window respectively. The finite element result for the quality factor for the cavity with beryllium windows is about $99.95 \%$ of the theoretical design value of the quality factor. Also, the finite element result for the quality factor for the cavity with the baseline gridded-tube window is about $99.95 \%$ of the theoretical design value of the quality factor. 
Fowler-Northam field emission model can be described by a current density $i$ that is given by [Fowler and Nordheim (1928)]

$$
i=\frac{A_{F N}\left(\beta E_{s}\right)^{2}}{\phi_{F N}} \exp \left(\frac{-B_{F N} \phi_{F N}^{3 / 2}}{\beta E_{s}}\right)
$$

where $E_{s}$ is the average surface electric field, $\phi$ is the work function of the material, $A_{F N}$ and $B_{F N}$ are constants, and $\beta$ is the electric field enhancement at the tip of the emitter. $\beta$ is defined as the ratio of the electric field at the tip of the emitter $\left(E_{m}\right)$ to the average surface electric field $\left(E_{s}\right)$. Assuming $E_{0}$ is the on-axis electric field and $\beta_{\text {tot }}$ is the total electric field enhancement, then the enhancements and the fields are related such that

$$
E_{m}=\beta_{t o t} E_{0}=\beta E_{s}
$$

It is important to distinguish between the enhancements presented in the previous equation and the surface electric field enhancement, $\zeta$, that has been discussed in the previous chapters. The surface electric field enhancement, $\zeta$, was defined as the ratio of the maximum surface electric field $\left(E_{s}^{\max }\right)$ to the on-axis electric field $\left(E_{0}\right)$. The enhancements are related such that

$$
\zeta=\beta_{t o t} / \beta
$$

[Norem et al. (2003)c] performed the first systematic study of an RF cavity subjected to high RF power and a strong magnetic field $(2.5-4.0) \mathrm{T}$. They studied dark current, breakdown, and magnetic field effects in a six cell, $805 \mathrm{MHz}$ cavity. Measurements of the dark current were in good agreement with the Fowler-Northam field emission model. The surface electric field enhancement, $\zeta$, was about 2.6. The ratio of the electric field at the tip of the emitter to the average surface electric field, $\beta$, was measured to be about 184 . The locally enhanced field intensities, $E_{m}$, at the tips of the emitters were found to be very large (about $10 \mathrm{GV} / \mathrm{m}$ ). The electromagnetic stresses exerted by the electric filed on the emitters were close to the tensile strength 
of the copper material. Damage in the titanium vacuum window, that was placed at the end of the cavity, due to dark current heating was reported. A digital photograph of the outer surface of the titanium vacuum window is shown in Figure A1. This figure shows an array of black spots that could be formed due to the local heating of the window by the dark current. A scanning electron microscope image of the inner surface of the titanium window is shown in Figure A2. This figure shows deposition of copper droplets on the surface of the window as a results of the role of field emission. The dark current was approximately proportional to the surface electric field raised to the tenth power. In this thesis, the ratio of the surface electric field enhancement, $\zeta$, varied from 1.11 to 1.95 . This indicates that for the test cavity closed by the beryllium foils or the gridded-tube windows, the dark current produced will be less than that for the open multi-cell cavity. 


\section{CHAPTER 7 \\ CONCLUSIONS AND RECOMMENDATIONS}

\subsection{Conclusions}

Electromagnetic, thermal and structural analyses of RF cavities were performed as part of a developmental RF cavity program for muon cooling. Closing the RF cavity ends by electrically conducting windows reduces the power requirement and considerably increases the on-axis electric field for a given maximum surface electric field. In this work, a finite element method for the electromagnetic and mechanical design of RF cavity windows is presented. The method is based on performing finite element analysis from electromagnetic, thermal, and structural points of view in an iterative manner. The finite element procedure involved several steps. First, the frequency, electric and magnetic fields, quality factor, and power loss density results were calculated. Then, the power loss density results were transferred from the electromagnetic mesh to the thermal mesh to calculate the temperature results. Next, the temperature results were transferred from the thermal mesh to the structural mesh to calculate the displacement and the thermal stresses. The procedure was applied to an $805 \mathrm{MHz}$ RF cavity for a variety of RF cavity window configurations. The configurations included thin, circular foils and thin-walled, gridded-tube patterns.

As a starting point in the analysis, a cylindrical pillbox cavity closed by thin, flat, beryllium windows was considered as a benchmark problem. Analytical and finite element solutions were obtained for the frequency, electric and magnetic fields, quality factor, power loss density, and temperature profile. Excellent agreement was obtained between the analytical and finite element results.

Gridded-tube windows, composed of thin-walled tubes arranged in specific patterns, were considered due to their high structural integrity. Three gridded-tube win- 
dow configurations were studied. The first configuration was a grid of non-touching tubes, composed of an array of tubes parallel to the x-axis and another array of tubes parallel to the y-axis, with a small gap between both arrays (see Figure 5.1). The second configuration was a grid of touching tubes, composed of an array of tubes parallel to the x-axis and another array of tubes parallel to the $\mathrm{y}$-axis, where both arrays are in contact with each other. The third configuration was a "waffle" grid, composed of an array of tubes parallel to the x-axis that is intersecting with another array of tubes parallel to the y-axis.

The window design should produce a uniform electric field distribution with negligible electric field distortion. Local field non-uniformity, and field distortion can affect the spread of the beam energy. In addition, the window design should terminate the electric field, or cause negligible electric field leakage to the regions outside the window. The field termination is necessary is because the closed-cell RF cavities should operate independently. For all the gridded-tube configurations, the electric field distribution was smooth in the cavity with small distortion in the vicinity of the grid region. It was found that the arrangement of the tubes controlled the field termination. For example, both of the "waffle" grid and the grid of touching tubes terminated the fields.

This thesis presented innovative ideas for decreasing the surface electric field enhancement in RF cavities by using RF cavity windows. The surface electric field enhancement was defined as the ratio of the maximum surface electric field to the on-axis electric field. In a field emission study for the open, multicell $805 \mathrm{MHz}$ test cavity, dark current was found to be proportional to the surface electric field raised to the tenth power [Norem at al. (2003c)]. So reducing this ratio serves to considerably reduce the dark current. For the open, multicell, $805 \mathrm{MHz}$ test cavity, the surface electric field enhancement was about 2.6. For the $805 \mathrm{MHz}$ test cavity closed 
by beryllium foils, the surface electric field enhancement was about 1.11. For the $805 \mathrm{MHz}$ test cavity with gridded tube windows, the surface electric field enhancement ranged between 1.31 to 1.95 depending on the grid pattern. It was concluded that increasing the number of tubes in the grid, increasing the tube outer diameter, and/or decreasing the spacing between the tubes in the middle of the grid reduces the surface electric field enhancement.

RF heating can cause the windows to deform out-of-plane. The out-of-plane deflection of the window must be kept below $25 \mu \mathrm{m}$ to prevent the frequency of the cavity from shifting more than $10 \mathrm{kHz}$. The thermal stresses in the window must also be maintained below the yield stress of the window material. The $4 \times 4$ "waffle" gridded-tube window, with a grid radius of $80.0 \mathrm{~mm}$, and a tube diameter of 9.5 $\mathrm{mm}$, was assumed to be the baseline gridded-tube window. For the baseline griddedtube window, with a grid wall thickness of $254 \mu \mathrm{m}$, the maximum temperature rise in the grid was $55.6^{\circ} \mathrm{C}$. The maximum displacement was $110 \mu \mathrm{m}$. The maximum von Mises stress was $245 \mathrm{MPa}$, and the yield stress safety factor was 1.12. Cooling this gridded-tube window by helium gas, at a convection heat transfer coefficient of $250 \mathrm{~W} / \mathrm{m}^{2} \cdot \mathrm{K}$, decreased the maximum temperature rise in the grid to $7.7^{\circ} \mathrm{C}$. The maximum displacement was also decreased to $13 \mu \mathrm{m}$. The maximum von Mises stress was decreased to $30.8 \mathrm{MPa}$, and the yield stress safety factor was increased to 8.96.

The gridded-tube RF cavity windows studied in this thesis are new designs that have not been previously considered. The present work shows that gridded-tube windows are feasible for use in RF cavity applications provided that the grid pattern, tube outer diameter, tube wall thickness, and other design parameters are chosen appropriately. 


\subsection{Recommendations for Future Work}

1) Field emission forces major limitations on a variety of RF applications. The electromagnetic stress exerted by the electric field on the emitter could be sufficient to pull out metallic particles and weld them to the surfaces. A technique is needed to help gain an understanding of the relationships between the surface electric field, material particles, and field emission.

2) This work presented innovative RF cavity windows such as the gridded-tube windows and demonstrated a procedure for their design. In the international muon cooling and ionization experiment (MICE), 201.25 MHz cavities were proposed for use as accelerating structures. It is recommended to study the design of gridded-tube windows for the RF cavities in MICE and other RF applications. 
APPENDIX A

DESCRIPTION OF THE DAMAGE IN THE TITANIUM WINDOW DUE TO FIELD EMISSION 


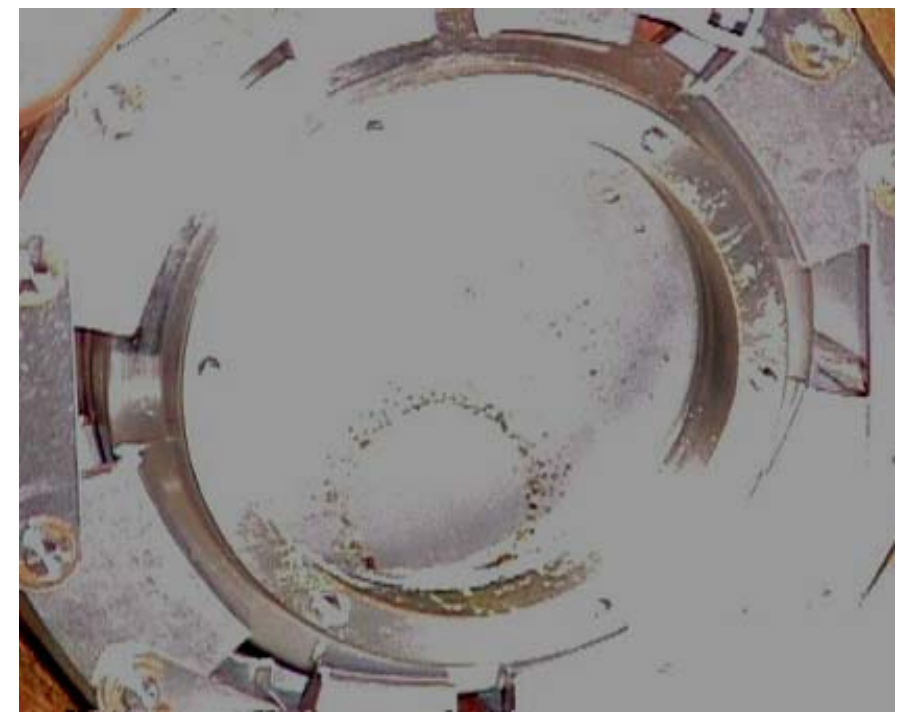

Figure A.1. Damage in the Titanium Vacuum Window Caused by Field Emission [Norem at al. (2003c)].

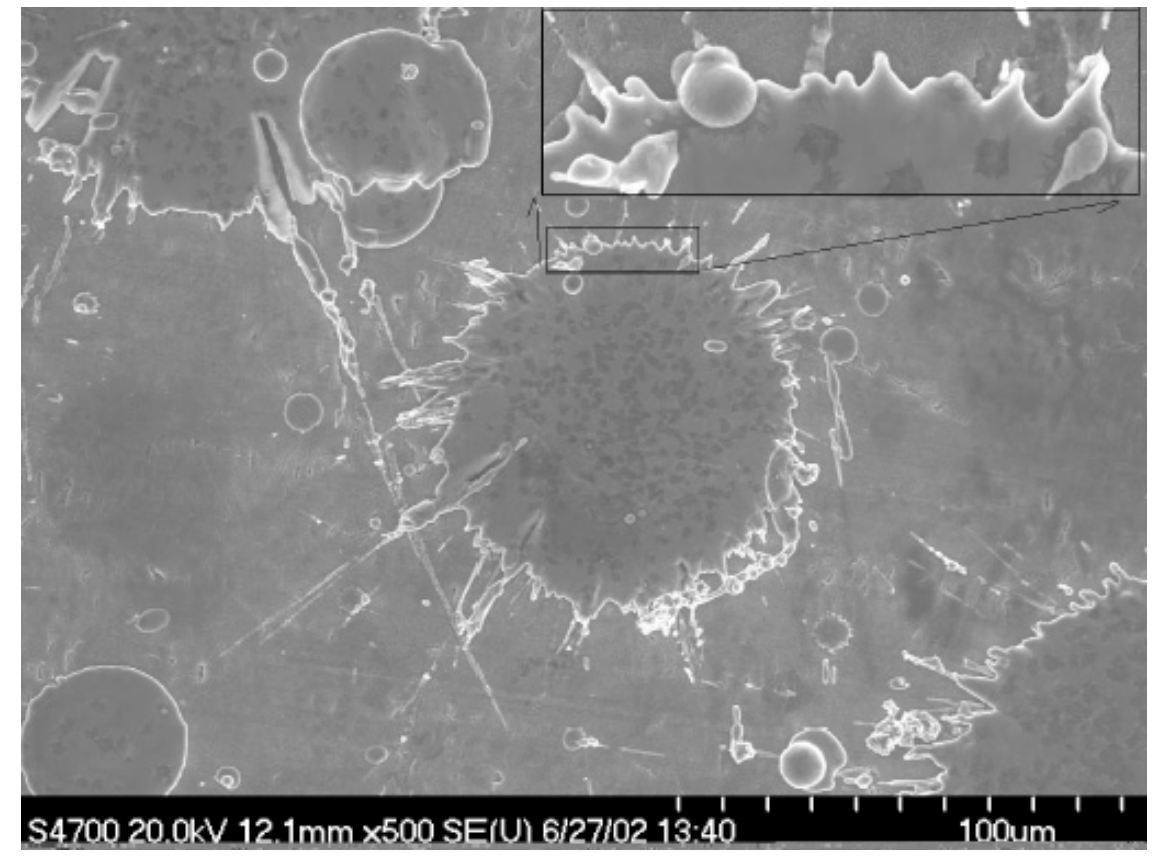

Figure A.2. Scanning Electron Microscope Image of Copper Splattered on the Inner Surface of the Titanium Window Caused by Field Emission ([Norem at al. (2003c)]. 
APPENDIX B

FINITE ELEMENT FORMULATION OF THE ELECTRIC FIELD INSIDE THREE DIMENSIONAL CAVITIES 
The governing equation for the electric field inside an RF cavity that is filled with a dielectric medium is given by

$$
\vec{\nabla} \times(\vec{\nabla} \times \vec{E})-\mu \epsilon \omega^{2} \vec{E}=0
$$

where $\vec{E}$ is the electric field, $\omega$ is the frequency of the cavity, $\mu$ is the magnetic permeability of the medium, and $\epsilon$ is the permittivity of the medium. At points on the inner surface of the cavity, the following condition must hold

$$
\vec{n} \times \vec{E}=0,
$$

where $\vec{n}$ is the unit outward normal to the inner surface of the cavity. If we multiply Equation (B.1) by a test function, $\vec{V}$, and integrate over the volume of the cavity, $v$, we get

$$
\int_{v}[\vec{V} \cdot(\vec{\nabla} \times(\vec{\nabla} \times \vec{E}))] \mathrm{d} v-\mu \epsilon \omega^{2} \int_{v}(\vec{V} \cdot \vec{E}) \mathrm{d} v=0 .
$$

Using the vector identity, $\vec{A} \cdot(\vec{\nabla} \times \vec{B})=(\vec{\nabla} \times \vec{A}) \cdot \vec{B}-\vec{\nabla} \cdot(\vec{A} \times \vec{B})$, Equation (B.3) can be written as

$$
\int_{v}(\nabla \times \vec{V}) \cdot(\vec{\nabla} \times \vec{E}) \mathrm{d} v=\mu \epsilon \omega^{2} \int_{v} \vec{V} \cdot \vec{E} \mathrm{~d} v+\int_{v} \vec{\nabla} \cdot[\vec{V} \times(\vec{\nabla} \times \vec{E})] \mathrm{d} v
$$

Using the divergence theorem, $\int_{v}(\vec{\nabla} \cdot \vec{A}) \mathrm{d} v=\int_{S}(\vec{A} \cdot \vec{n}) \mathrm{d} S$, Equation (B.4) becomes

$$
\int_{v}(\vec{\nabla} \times \vec{V}) \cdot(\vec{\nabla} \times \vec{E}) \mathrm{d} v=\mu \epsilon \omega^{2} \int_{v} \vec{V} \cdot \vec{E} \mathrm{~d} v-\int_{S} \vec{V} \cdot[\vec{n} \times(\vec{\nabla} \times \vec{E})] \mathrm{d} S
$$

where $S$ is the inner surface of the cavity. The boundary condition given by (B.2) requires that the last term in Equation (B.5) vanish. Equation (B.5) becomes,

$$
\int_{v}(\nabla \times \vec{V}) \cdot(\vec{\nabla} \times \vec{E}) \mathrm{d} v=\mu \epsilon \omega^{2} \int_{v} \vec{V} \cdot \vec{E} \mathrm{~d} v
$$

For the 6-noded tetrahedral element shown in Figure B.1, The electric field can be represented as [see e.g. Reddy et al. (1994)],

$$
\vec{E}=\sum_{m=1}^{6} e_{m} \vec{N}_{m}
$$


where $m$ stands for the edge number, $e_{m}$ are the magnitudes of the electric field along each edge, and $\vec{N}_{m}$ are the vector tangential edge elements given by [Bossavit (1989)] as

$$
\vec{W}_{N}=L_{m}\left(\alpha_{t i} \vec{\nabla} \alpha_{t j}-\alpha_{t j} \vec{\nabla} \alpha_{t i}\right)
$$

where $i$ and $j$ stands for the nodes connecting the edge $m, L_{m}$ is the length of the edge $m, \alpha_{t i}$ and $\alpha_{t j}$ are the simplex coordinate associated with nodes $i$ and $j$.

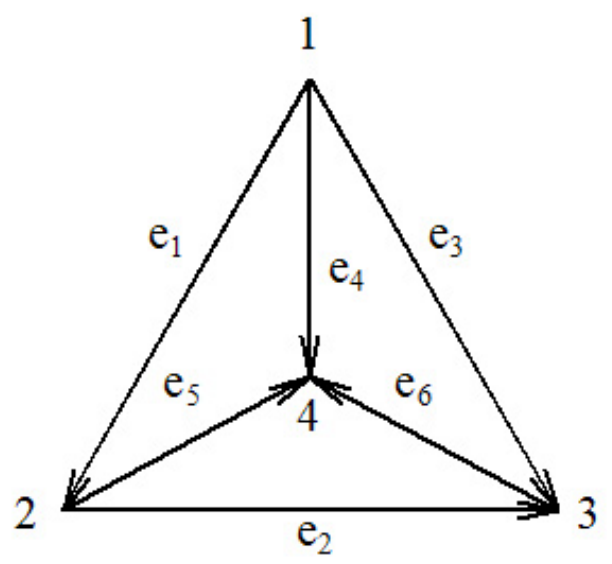

Figure B.1. Tetrahedral Element with Four Nodes and Six Edges.

Finally, upon the substitution of Equation (B.7) into Equation (B.6), and integrating over the volume of a single tetrahedral element, we get

$$
\sum_{m=1}^{6} \int_{v}\left(\vec{\nabla} \times \vec{N}_{m}\right) \cdot\left(\vec{\nabla} \times \vec{N}_{n}\right) e_{m} \mathrm{~d} v=\mu \epsilon \omega^{2} \sum_{m=1}^{6} \int_{v}\left(\vec{N}_{m} \cdot \vec{N}_{n}\right) e_{m} \mathrm{~d} v
$$

Equation (B.6) can be rewritten as

$$
\left[S_{e l}\right][e]=\omega^{2}\left[T_{e l}\right][e]
$$

where $\left[S_{e l}\right]$ is given by

$$
\left[S_{e l}\right]=\int_{v}\left(\vec{\nabla} \times \vec{N}_{m}\right) \cdot\left(\vec{\nabla} \times \vec{N}_{n}\right) e_{m} \mathrm{~d} v
$$


and $\left[T_{e l}\right]$ is given by

$$
\left[T_{e l}\right]=\mu \epsilon \sum_{m=1}^{6} \int_{v}\left(\vec{N}_{m} \cdot \vec{N}_{n}\right) e_{m} \mathrm{~d} v .
$$

The electric field inside the cavity is then obtained by assembling the element matrices over all the tetrahedral elements such that

$$
[S][e]=\omega^{2}[T][e] .
$$

The eigenvalues can be extracted using a standard lancos algorithm [see e.g. Bathe (2004)]. 


\section{BIBLIOGRAPHY}

[Alsharo'a et al. (2003)] Alsharo'a M., et al. "Recent Progress in Neutrino Factory and Muon Collider Research Within the Muon Collaboration." Phys. Rev. ST $A B \quad 6$ (2003): 1-52.

[ANSYS (2003)] "ANSYS Finite Element Software." ANSYS Inc. Corporate. www.ansys.com.

[Bathe (2004)] Bathe, K. Finite Element Procedures. New Delhi: Printice-Hall of India, 2004.

[Bossavit (1989)] Bossavit, A. "Simplicial Finite Elements for Scattering Problems in Electromagnetism." Computational Methods in Applied Mechanics and Engineering 76 (1989): 299-316.

[Corlett at al. (1999)] Corlett, J. N., Li, D., and MacGill, R. A. "RF Accelerating Structures for the Muon Cooling Experiment." Proceedings of the 1999 Particle Accelerator Conference. Ed. Luccio A. New York: IEEE, 1999. 3149-3151.

[Fowler and Nordheim (1928)] Fowler, R. H., and Nordheim, L. "Electron Emission in Intense Electric Fields." Proc. Roy. Soc. A 119 (1928): 173-181.

[Hartman et al. (2000)] Hartman, N., Li, D., and Corlett, J. N. "Comparison of Deflection Measurements to FEA Modeling for Muon Cooling Channel RF Cavity Windows." Muon Cooling Note Number 111. Muon Collider Collaboration, Fermilab, Batavia IL, 2000.

[Hartman et al. (2001)] Hartman, N., Corlett, J. N., and Li, D. "Finite Element Analysis of Thin Beryllium Windows for a Muon Cooling Channel." Proceedings of the 2001 Particle Accelerator Conference. Ed. Lucas P. Chicago: IEEE, 2001. 909-911.

[Jackson (1998)] Jackson, J. D. Classical Electrodynamics. 2nd ed. New York: John Wiley \& Sons, 1998.

[Kaplan (2001)] Kaplan, D. M. "Introduction to Muon Cooling." Snowmass Summer Studies 2001 Proceedings. Ed. Graf N. Snowmass: APS, 2001. M102 1-9.

[Li et al. (2001)] Li, D., Corlett, J. N., and MacGill, R. A. "Design and Fabrication of an $805 \mathrm{MHz}$ RF Cavity with Beryllium Windows for a High Power RF Testing for a Muon Cooling Experiment." Proceedings of the 2001 Particle Accelerator Conference. Ed. Lucas P. Chicago: IEEE, 2001. 918-920.

[Li et al. (2002)a] Li, D., Corlett, J. N., and MacGill, R. A. "High Power RF Test for an $805 \mathrm{MHz}$ RF Cavity for a Muon Cooling Channel." Proceedings of the Europian Particel Accelerator Confereence 2002. Paris, 2002. 2160-2162.

[Li et al. (2002)b] Li, D., Ladran, A., and Lozano, D. "Mechanical and Thermal Analysis of Beryllium Windows for RF Cavities in a Muon Cooling Channel." Proceedings of the Europian Particel Accelerator Conference 2002. Paris, 2002. 2163-2165.

[Li et al. (2003)a] Li, D., Corlett, J. N., and Ladran, A. "805 MHz and $201 \mathrm{MHz}$ RF Cavity Development for MUCOOL." J. Phys. G: Nucl. Part. Phys. 29 (2003): 1683-1687. 
[Li et al. (2003)b] Li, D., Ladran, A., and Staples, J. "A 201 MHz RF Cavity Design with Non-Stressed and Pre-Curved Be Windows for Muon Cooling Channels." Proceedings of the 2003 Particle Accelerator Conference. Ed. Chew J. Portland: IEEE, 2003. 1243-1245.

[Moretti et al. (2000)] Moretti, A., Holtkamp, N., and Jurgens, T. "RF Cavities for the Muon and Neutrino Factory Collaboration Study." XX International Linac Conference Proceedings. Ed. Chao A. Monterey, 2000. 896-898.

[Norem et al. (2003)a] Norem, J., Gruber, P., and Bross, A. "Dark Current and X Ray Measurements of an $805 \mathrm{MHz}$ Pillbox Cavity." Proceedings of the 2003 Particle Accelerator Conference. Ed. Chew J. Portland: IEEE, 2003. 1183-1185.

[Norem et al. (2003)b] Norem, J., Li, D., and Bross, A. "RF Induced Backgrounds in Muon Ionization Cooling Experiment." J. Phys. G: Nucl. Part. Phys 29 (2003): 1697-1700.

[Norem at al. (2003c)] Norem, J., Wu, V., and Moretti, A. "Dark Current, Breakdown, and Magnetic Field Effects in a Multicell, $805 \mathrm{MHz}$ Cavity." Phys. Rev. ST AB 6 (2003): 1-21.

[Reddy et al. (1994)] Reddy, C. J., Deshpande, M. D., and Cockrell, C. R., "Finite Element Method for Eigenvalue Problems in Electromagnetics." NASA Technical Paper 3485. NASA Center for Aerospace Information. Linthicum Heights MD, 1994.

[Rimmer et al. (2001)] Rimmer, R. A., Hartman, N., and Ladran, A. "Closed cell 201.25 MHz RF Structures For a Muon Cooling Channel." Proceedings of the 2001 Particle Accelerator Conference. Ed. Lucas P. Chicago: IEEE, 2001. 927929.

[Schiff (1990)] Schiff, D. Dynamic Analysis and Failure Modes of Simple Structures. New York: John Wiley \& Sons, 1990.

[Turner et al. (1998)] Turner, W. C., Moretti, A., and Corlett, J. N. "RF System Concepts for a Muon Cooling Experiment." Sixth European Particle Accelerator Conference Proceedings. Ed. Myers S. Stockholm: Institute of Physics Publishing-Bristol and Philadelphi, 1998. 371-373.

[Wu (2002)] Wu, V. "Design and Testing of a High Gradient Radio Frequency Cavity for the Muon Collider." Ph.D. Thesis. Univeristy of Cincinnati, 2002. 